Supporting Information

\title{
QM/MM Study of Mechanisms of Heme Degradation by the Enzyme Heme Oxygenase: The Strategic Function of the Water Cluster
}

\author{
Hui Chen, Yohann Moreau, Etienne Derat, ${ }^{\#}$ Sason Shaik* \\ Department of Organic Chemistry and the Lise Meitner-Minerva Center for \\ Computational Quantum Chemistry, The Hebrew University of Jerusalem, Givat Ram \\ Campus, 91904 Jerusalem, Israel \\ ${ }^{\#}$ Current address: Laboratoire de Chimie Organique, Institut de Chimie Moléculaire, \\ Université Pierre et Marie Curie-Paris 6, 4 place Jussieu B. 229, 75505 Paris, France
}




\section{Computational Details}

QM/MM calculations have been done with the Chemshell application combining Turbomole/DL-POLY. The hybrid B3LYP functional was used throughout this study for the QM part, and CHARMM22 force field was used for the MM part. Two basis sets, B1 and B2, as described below, were used for QM part of the system. B1 is for geometry optimization and the much larger B2 basis is used only for single point calculations.

B1: $6-31+\mathrm{G}(\mathrm{d})$ for $\mathrm{O}_{2}$ coordinated with iron and double- $\zeta$ basis set LACVP for all other atoms.

B2: Wachter all electron basis of for $\mathrm{Fe}, 6-31+\mathrm{G}^{*}$ for all electronegative atoms $(\mathrm{O}$ and $\mathrm{N}), 6-31 \mathrm{G}(\mathrm{d}, \mathrm{p})$ for $\mathrm{C}$ and $\mathrm{H}$ atoms.

Standard CHARMM22 force field was used for the MM part.

\section{QM/MM preparation:}

To prepare suitable initial structures for the QM/MM calculatuions, we started from the experimental X-ray structure of dioxygen-bound HmuO (PDB code 1V8X). There are three equivalent subunits, namely $\mathrm{A}, \mathrm{B}$ and $\mathrm{C}$ in the $\mathrm{X}$-ray structure and we chose the B-subunit for our QM/MM calculations. We built a complete model of solvated enzyme by adding the missing hydrogen atoms and a $16-\AA$-thick water solvent layer. The system was prepared according to the following standard procedures. First, the missing hydrogen atoms were added. Second, the added hydrogen atoms were optimized. Third, the enzyme was soaked in a 16 - $\AA$-thick water solvent layer. Fourth, the inner 8 - $\AA$-thick solvent layer was minimization-equilibration-minimization scheme while keeping the remainder of the system fixed. The procedure of adding the solvent was repeated once to ensure that not more than 100 additional water molecules were added at last. The entire system consists of 15273 atoms, including 11292 atoms in the solvent. This system was then relaxed by performing pure force field minimizations and 300 ps molecular dynamics (MD), with the CHARMM22 force field as implemented in the CHARMM program, during which the coordinates of the heme, the proximal coordinating His20 and the outer 8 - $\AA$-thick water solvent layer were kept fixed. After this MD simulation, we pick up two additional snapshots (after 116 and 287 ps of equilibration) and with snapshot at 0 ps. These three snapshots form the basis of our QM/MM calculation in this work.

\section{Snapshot 0 ps:}

QM part: iron-hydroperoxo-porphyrin, His20 (proximal ligand, modeled as an imidazole), distal residue Arg132 (modeled as $\mathrm{NH}_{2} \mathrm{C}^{+}\left(\mathrm{NH}_{2}\right)_{2}$ ), Asp136 (modeled as $\mathrm{HCOO}^{-}$), Wat6, Wat19, Wat61, Wat160, Wat219, Wat375.

During QM/MM geometry optimization, the following residues were optimized: Enzyme residues Lys13, Thr16, Ala19, His20 (including iron-hydroperoxo-porphyrinIX), Glu21, Ala23, Glu24, Met29, Leu33, Gln46, Tyr53, Tyr130, Val131, Arg132, Tyr133, Leu134, Gly135, Asp136, Leu137, Ser138, Gly139, Gly140, Gln141, Val142, Ile143, 
Ala144, Phe160, Tyr161, Arg177, Phe201, Phe208.

Crystal water Wat6, Wat19, Wat39, Wat46, Wat61, Wat71, Wat134, Wat160, Wat219, Wat226, Wat375, Wat381.

Solvent water 565, 728, 739, 815, 841, 883, 884, 885, 917, 922.

\section{Snapshot 116 ps:}

QM part: iron-hydroperoxo-porphyrin, His20 (proximal ligand, modeled as an imidazole), distal residue $\mathrm{Arg} 132$ (modeled as $\mathrm{NH}_{2} \mathrm{C}^{+}\left(\mathrm{NH}_{2}\right)$ ), Asp136 (modeled as $\mathrm{HCOO}^{-}$), Wat6, Wat19, Wat61, Wat71, Wat134, Wat219, Wat375.

During QM/MM geometry optimization, the following residues were optimized:

Enzyme residues Lys13, Thr16, Ala19, His20 (including iron-hydroperoxo-porphyrinIX), Glu21, Ala23, Glu24, Met29, Leu33, Gln46, Tyr53, Tyr130, Val131, Arg132, Tyr133, Leu134, Gly135, Asp136, Leu137, Ser138, Gly139, Gly140, Gln141, Val142, Ile143, Ala144, Phe160, Tyr161, Arg177, Phe201, Phe208.

Crystal water Wat6, Wat19, Wat39, Wat46, Wat61, Wat71, Wat134, Wat160, Wat219, Wat226, Wat375.

Solvent water 753, 766, 818, 880, 885, 887, 889, 909, 917, 918, 922, 934, 954, 1214.

\section{Snapshot 287 ps:}

QM part: iron-hydroperoxo-porphyrin, His20 (proximal ligand, modeled as an imidazole), distal residue Arg132 (modeled as $\mathrm{NH}_{2} \mathrm{C}^{+}\left(\mathrm{NH}_{2}\right)$ ), Asp136 (modeled as $\mathrm{HCOO}^{-}$), Wat6, Wat19, Wat61, Wat71, Wat134, Wat160, Wat219.

During QM/MM geometry optimization, the following residues were optimized:

Enzyme residues Lys13, Thr16, Ala19, His20 (including iron-hydroperoxo-porphyrinIX), Glu21, Ala23, Glu24, Met29, Leu33, Gln46, Tyr53, Tyr130, Val131, Arg132, Tyr133, Leu134, Gly135, Asp136, Leu137, Ser138, Gly139, Gly140, Gln141, Val142, Ile143, Ala144, Phe160, Tyr161, Arg177, Phe201, Phe208.

Crystal water Wat6, Wat19, Wat39, Wat46, Wat61, Wat71, Wat134, Wat160, Wat219, Wat226, Wat261, Wat375, Wat381.

Solvent water 174, 565, 749, 818, 835, 867, 870, 884, 885, 914, 917, 922, 934, 954.

For the iron hydrogen peroxide species, the Cpd 0 structures of corresponding snapshots were used with a proton added to the proximal oxygen atom. Appropriate changes were made to the parameter and topology files. 


\section{Full References}

\section{Ref. 27 in Full}

Sherwood, P.; de Vries, A. H.; Guest, M. F.; Schreckenbach, G.; Catlow,C. R. A.; French, S. A.; Sokol, A. A.; Bromley, S. T.; Thiel, W.; Turner, A. J.; Billeter, S.; Terstegen, F.; Thiel, S.; Kendrick, J.; Rogers, S. C.; Casci, J.; Watson, M.; King, F.; Karlsen, E; Sjovoll, M.; Fahmi, A.; Schäfer, A.; Lennartz, C. J. Mol. Struc. (Theochem) 2003, 632, 1.

\section{Ref. 31 in Full}

MacKerell, A. D., Jr.; Bashford, D.; Bellott, R. L.; Dunbrack, R. L., Jr.; Evanseck, J. D.; Field, M. J.; Fischer, S.; Gao, J.; Guo, H.; Ha, S.; Joseph-McCarthy, D.; Kuchnir, L.; Kuczera, K.; Lau, F. T. K.; Mattos, C.; Michnick, S.; Ngo, T.; Nguyen, D. T.; Prodhom, B.; Reiher, W. E., III; Roux, B.; Schlenkrich, M.; Smith, J. C.; Stote, R.; Straub, J.; Watanabe, M.; Wiorkiewicz-Kuczera, J.; Yin, D.; Karplus, M. J. Phys. Chem. B 1998, $102,3586$. 


\section{PES Scan results}

\section{Snapshot 0 ps}

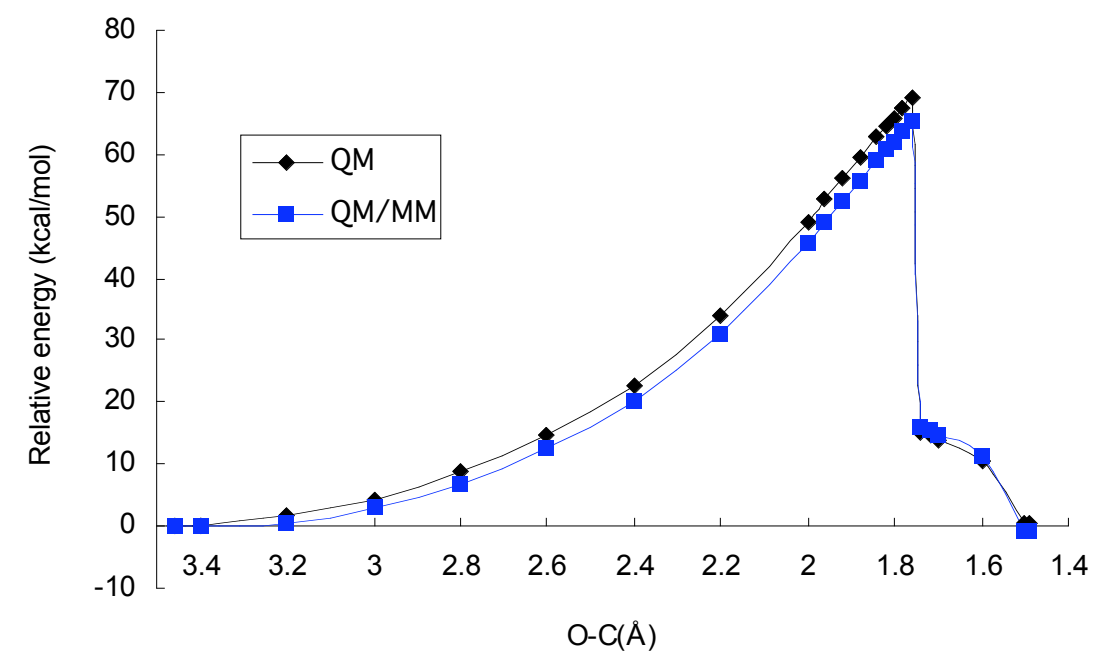

Figure S1. The scan energy profile along the $\mathrm{O}-\mathrm{C}_{\alpha \text {-meso }}$ from ${ }^{2} \mathbf{C}_{\mathrm{R}}$ at the $\mathrm{QM} / \mathrm{MM} \mathrm{B} 1$ level for the 0 ps snapshot. ${ }^{2} C_{R}$ was taken as zero point in energy. This scan is for exploring the concerted $\mathrm{O}-\mathrm{O}$ bond breaking and $\mathrm{O}_{\text {distal }}-\mathrm{C}_{\alpha \text {-meso }}$ bond formation from $\mathrm{Cpd} 0$.

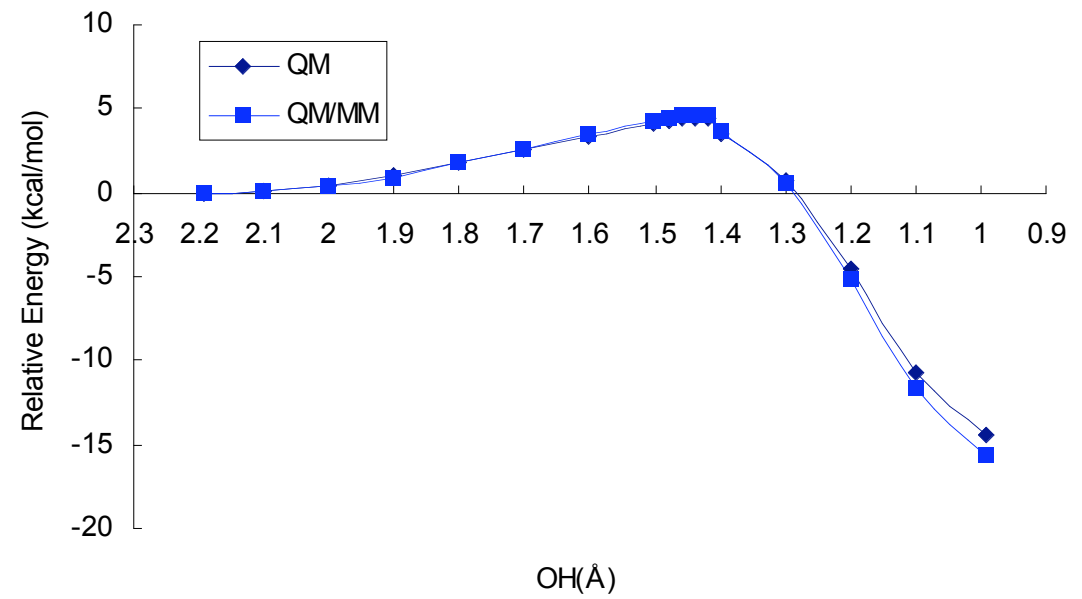

Figure S2. The scan energy profile along the $\mathrm{O}_{\text {distal }}-\mathrm{H}_{\text {proximal }}$ forming bond from ${ }^{2} \mathbf{C}_{\mathrm{Ia}} \mathbf{H}$ to Cpd I at the QM/MM B1 level for the 0 ps snapshot. ${ }^{2} \mathbf{C}_{\mathrm{Ia}} \mathbf{H}$ was taken as zero point in energy. 


\section{Snapshot 116 ps}

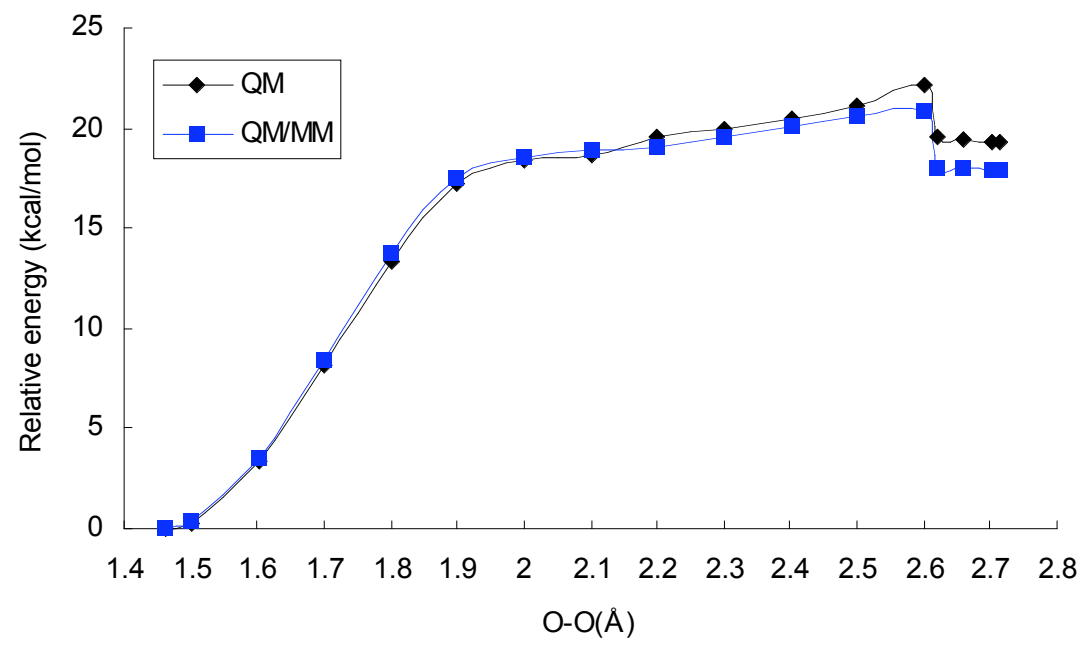

Figure S3. The scan energy profile along the breaking O-O bond from ${ }^{2} \mathbf{C}_{\mathrm{R}}$ at the $\mathrm{QM} / \mathrm{MM}$ B1 level for the $116 \mathrm{ps}$ snapshot. ${ }^{2} \mathbf{C}_{\mathrm{R}}$ was taken as zero point in energy.

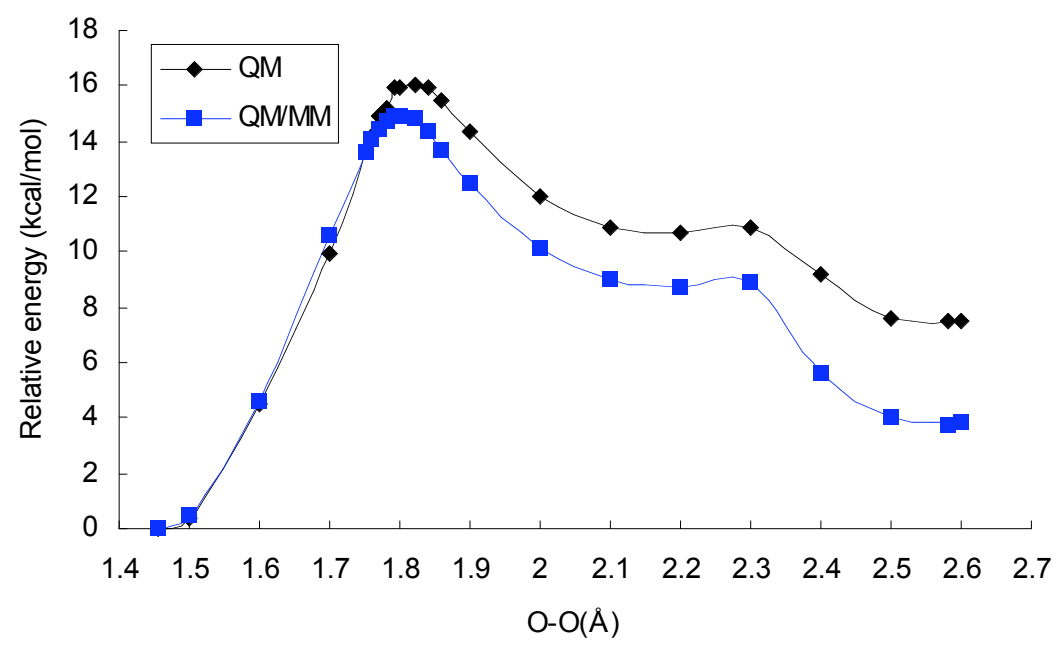

Figure S4. The scan energy profile along the breaking O-O bond from ${ }^{2} \mathbf{C}_{\mathrm{R}} \mathbf{H}$ at the $\mathrm{QM} / \mathrm{MM}$ B1 level for the 116 ps snapshot. ${ }^{2} \mathbf{C}_{\mathrm{R}} \mathbf{H}$ was taken as zero point in energy. 


\section{Snapshot 287 ps}

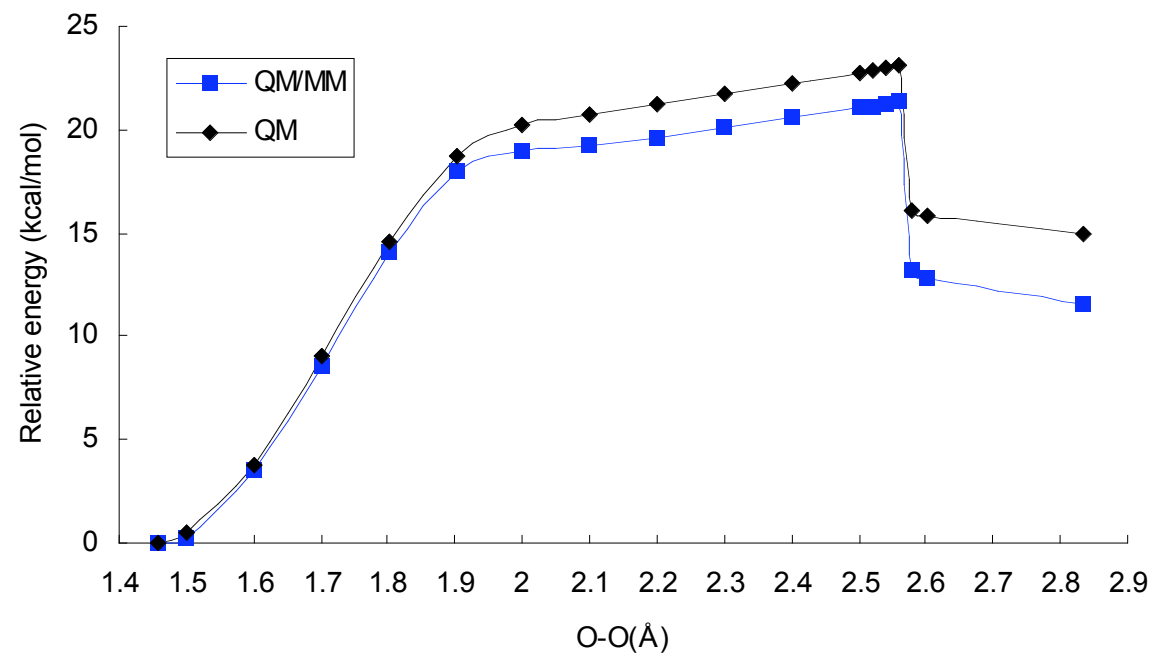

Figure S5. The scan energy profile along the breaking O-O bond from ${ }^{2} \mathbf{C}_{\mathrm{R}}$ at the $\mathrm{QM} / \mathrm{MM}$ B1 level for the $287 \mathrm{ps}$ snapshot. ${ }^{2} \mathbf{C}_{\mathrm{R}}$ was taken as zero point in energy.

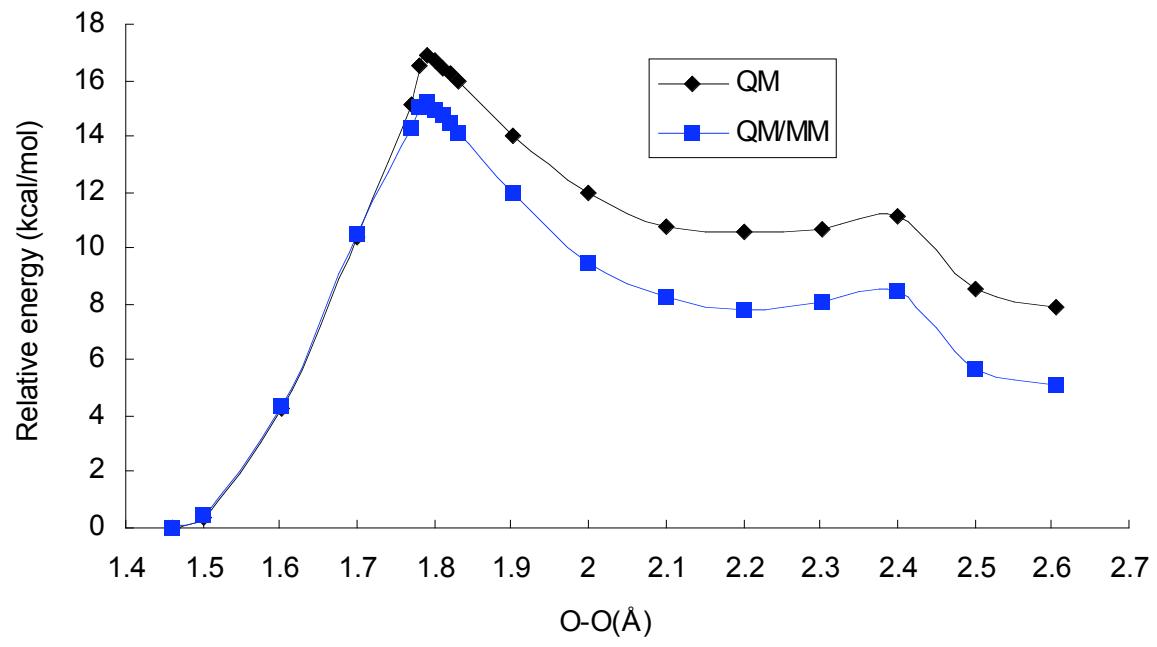

Figure S6. The scan energy profile along the breaking O-O bond from ${ }^{2} \mathbf{C}_{\mathrm{R}} \mathbf{H}$ at the QM/MM B1 level for the 287 ps snapshot. ${ }^{2} \mathbf{C}_{\mathrm{R}} \mathbf{H}$ was taken as zero point in energy. 


\section{Geometries}

Table S1. Key Geometrical Parameters (Bond Distance in $\AA$ and Bond Angle in Degree) of the Three Spin States of Cpd 0.

\begin{tabular}{|l|c|c|c|c|c|c|c|c|c|c|c|c|}
\hline & \multicolumn{3}{|c|}{$\mathrm{R}\left(\mathrm{Fe}-\mathrm{N}_{\text {ImH }}\right)$} & \multicolumn{3}{c|}{$\mathrm{R}(\mathrm{Fe}-\mathrm{O})$} & \multicolumn{3}{c|}{$\mathrm{R}(\mathrm{O}-\mathrm{O})$} & $\mathrm{R}_{\text {av }}\left(\mathrm{Fe}-\mathrm{N}_{\text {por }}\right)$ \\
\hline Snapshot & ${ }^{2} \mathbf{C}_{\mathrm{R}}$ & ${ }^{4} \mathbf{C}_{\mathrm{R}}$ & ${ }^{6} \mathbf{C}_{\mathrm{R}}$ & ${ }^{2} \mathbf{C}_{\mathrm{R}}$ & ${ }^{4} \mathbf{C}_{\mathrm{R}}$ & ${ }^{6} \mathbf{C}_{\mathrm{R}}$ & ${ }^{2} \mathbf{C}_{\mathrm{R}}$ & ${ }^{4} \mathbf{C}_{\mathrm{R}}$ & ${ }^{6} \mathbf{C}_{\mathrm{R}}$ & ${ }^{2} \mathbf{C}_{\mathrm{R}}$ & ${ }^{4} \mathbf{C}_{\mathrm{R}}$ & ${ }^{6} \mathbf{C}_{\mathrm{R}}$ \\
\hline $0 \mathrm{ps}$ & 2.039 & 2.243 & 2.211 & 1.838 & 2.021 & 1.988 & 1.461 & 1.460 & 1.448 & 2.035 & 2.041 & 2.092 \\
\hline $116 \mathrm{ps}$ & 2.048 & 2.339 & 2.286 & 1.828 & 2.014 & 1.973 & 1.463 & 1.458 & 1.443 & 2.033 & 2.037 & 2.091 \\
\hline $287 \mathrm{ps}$ & 2.037 & 2.257 & 2.240 & 1.819 & 1.993 & 1.966 & 1.458 & 1.453 & 1.440 & 2.033 & 2.039 & 2.091 \\
\hline & \multicolumn{3}{|c|}{$\mathrm{R}\left(\mathrm{O}_{\text {distal }}-\mathrm{H}_{\text {wat }}\right)^{\mathrm{a}}$} & \multicolumn{3}{|c|}{$\angle \mathrm{FeOO}$} & \multicolumn{3}{c|}{$\mathrm{R}\left(\mathrm{N}_{\text {por }}-\mathrm{H}_{\text {distal }}\right)$} & \multicolumn{3}{c|}{$\mathrm{R}\left(\mathrm{O}_{\text {distal }}-\mathrm{C}_{\alpha-\text { meso }}\right)$} \\
\hline $0 \mathrm{ps}$ & 1.817 & 1.767 & 1.794 & 114.98 & 115.21 & 116.19 & 2.028 & 2.12 & 2.065 & 3.461 & 3.488 & 3.455 \\
\hline $116 \mathrm{ps}$ & 1.841 & 1.757 & 1.792 & 117.17 & 118.76 & 118.83 & 1.969 & 2.087 & 2.046 & 3.752 & 3.812 & 3.791 \\
\hline $287 \mathrm{ps}$ & 1.824 & 1.742 & 1.787 & 116.13 & 117.29 & 117.71 & 1.985 & 2.085 & 2.046 & 3.654 & 3.727 & 3.695 \\
\hline
\end{tabular}

${ }^{\mathrm{a}}$ Hydrogen bond distance between the distal $\mathrm{O}$ atom and the $\mathrm{H}$ atom of the water of the water cluster in distal pocket.

Table S2. Key Geometrical Parameters (Bond Distance in $\AA$ and Bond Angle in Degree) of the Three Spin States of FeHOOH.

\begin{tabular}{|c|c|c|c|c|c|c|c|c|c|c|c|c|}
\hline & \multicolumn{3}{|c|}{$\mathrm{R}\left(\mathrm{Fe}-\mathrm{N}_{\mathrm{ImH}}\right)$} & \multicolumn{3}{|c|}{$\mathrm{R}(\mathrm{Fe}-\mathrm{O})$} & \multicolumn{3}{|c|}{$\mathrm{R}(\mathrm{O}-\mathrm{O})$} & \multicolumn{3}{|c|}{$\mathrm{R}_{\mathrm{av}}\left(\mathrm{Fe}-\mathrm{N}_{\mathrm{por}}\right)$} \\
\hline Snaps & ${ }^{2} \mathbf{C}_{\mathrm{R}} \mathbf{H}$ & ${ }^{4} \mathbf{C}_{\mathrm{R}} \mathbf{H}$ & ${ }^{6} \mathbf{C}_{\mathrm{R}} \mathbf{H}$ & ${ }^{2} \mathbf{C}_{\mathrm{R}} \mathbf{H}$ & ${ }^{4} \mathbf{C}_{\mathrm{R}} \mathbf{H}$ & ${ }^{6} \mathbf{C}_{\mathrm{R}} \mathbf{H}$ & $\mathbf{C}_{\mathrm{R}} \mathbf{H}$ & ${ }^{4} \mathbf{C}_{\mathrm{R}} \mathbf{H}$ & ${ }^{6} \mathbf{C}_{\mathrm{R}} \mathbf{H}$ & ${ }^{2} \mathbf{C}_{\mathrm{R}} \mathbf{H}$ & ${ }^{4} \mathbf{C}_{\mathrm{R}} \mathbf{H}$ & ${ }^{6} \mathbf{C}_{\mathrm{R}} \mathbf{H}$ \\
\hline $0 \mathrm{ps}$ & 1.912 & 2.077 & 20 & & & & & & & 25 & 22 & 81 \\
\hline $116 \mathrm{ps}$ & 926 & 2.12 & 2.0 & & & & 57 & 5 & & 17 & & 2.070 \\
\hline \multirow[t]{2}{*}{$287 \mathrm{ps}$} & 24 & 2.113 & 2.0 & & $2.0 \angle 0$ & & 451 & 1.449 & 1.4 & 2.019 & 2.019 & 2.070 \\
\hline & \multicolumn{3}{|c|}{$\mathrm{R}\left(\mathrm{O}_{\text {distal }}-\mathrm{H}_{\text {wat }}\right)^{\mathrm{a}}$} & \multicolumn{3}{|c|}{$\angle \mathrm{FeOO}$} & \multicolumn{3}{|c|}{$\mathrm{R}\left(\mathrm{N}_{\text {por }}-\mathrm{H}_{\text {distal O }}\right)$} & \multicolumn{3}{|c|}{$\mathrm{R}\left(\mathrm{O}_{\text {distal }}-\mathrm{C}_{\alpha-\text { meso }}\right)$} \\
\hline $0 \mathrm{ps}$ & 1.977 & 1.7 & & & & & 1.857 & 2.096 & & 3.473 & & 3.439 \\
\hline & 1.949 & & & & & & & & & 3.805 & & 3.854 \\
\hline $287 \mathrm{ps}$ & 2.020 & 1.904 & 1.928 & 115.42 & 115.29 & 114.54 & 1.981 & 2.247 & 2.135 & 3.604 & 3.612 & 3.599 \\
\hline
\end{tabular}

${ }^{\mathrm{a}}$ Hydrogen bond distance between the distal $\mathrm{O}$ atom and the $\mathrm{H}$ atom of the water of the water cluster in distal pocket. 


\section{Energies}

Table S3. The Calculated Energies (a. u.) from the 0 ps Snapshot

\begin{tabular}{|l|c|c|c|c|}
\hline Energies & \multicolumn{2}{|c|}{$\mathrm{B} 1$} & \multicolumn{2}{c|}{$\mathrm{B} 2$} \\
\hline & QM & QM/MM & QM & QM/MM \\
\hline${ }^{2} \mathbf{C}_{\mathrm{R}}$ & -2342.579429 & -2368.925887 & -3483.551888 & -3509.898366 \\
\hline${ }^{4} \mathbf{C}_{\mathrm{R}}$ & -2342.562329 & -2368.906335 & -3483.537151 & -3509.881097 \\
\hline${ }^{6} \mathbf{C}_{\mathrm{R}}$ & -2342.556951 & -2368.903861 & -3483.533809 & -3509.880684 \\
\hline${ }^{2} \mathbf{C}_{\mathrm{Ia}}$ & -2342.541789 & -2368.888833 & -3483.519462 & -3509.866466 \\
\hline${ }^{2} \mathbf{C}_{\mathrm{Ib}}$ & -2342.547061 & -2368.893790 & -3483.525485 & -3509.872157 \\
\hline${ }^{2} \mathbf{P}$ & -2342.578732 & -2368.927242 & -3483.562133 & -3509.910587 \\
\hline${ }^{4} \mathbf{C}_{\mathrm{R}} \mathbf{H}$ & -2343.262026 & -2369.613108 & -3484.229545 & -3510.580529 \\
\hline${ }^{2} \mathbf{C}_{\mathrm{R}} \mathbf{H}$ & -2343.249450 & -2369.607249 & -3484.218289 & -3510.576119 \\
\hline${ }^{6} \mathbf{C}_{\mathrm{R}} \mathbf{H}$ & -2343.243002 & -2369.598845 & -3484.217178 & -3510.573104 \\
\hline${ }^{2} \mathbf{T S} \mathbf{S}_{\mathbf{O O}} \mathbf{H}$ & -2343.226633 & -2369.585552 & -3484.199701 & -3510.558557 \\
\hline${ }^{2} \mathbf{C}_{\mathrm{Ia}} \mathbf{H}$ & -2343.239807 & -2369.598167 & -3484.217783 & -3510.576157 \\
\hline${ }^{2} \mathbf{C}_{\mathrm{Ib}} \mathbf{H}$ & -2343.245772 & -2369.604066 & -3484.223560 & -3510.581815 \\
\hline${ }^{2} \mathbf{P H}$ & -2343.305577 & -2369.665559 & -3484.288049 & -3510.648034 \\
\hline ASPP136 ${ }^{2} \mathbf{C}_{\mathrm{R}}{ }^{\mathrm{a}}$ & -2343.224519 & -2369.584600 & -3484.192209 & -3510.552169 \\
\hline ASPP136 ${ }^{2} \mathbf{P}^{\mathrm{a}}$ & -2343.232085 & -2369.590852 & -3484.209351 & -3510.568010 \\
\hline
\end{tabular}

${ }^{\mathrm{a}}$ Asp136 is protonated here.

Table S4. The Calculated Relative Energies ( $\mathrm{kcal} / \mathrm{mol})$ from the 0 ps Snapshot

\begin{tabular}{|l|c|c|c|c|}
\hline Energies & \multicolumn{2}{|c|}{ B1 } & \multicolumn{2}{c|}{ B2 } \\
\hline & QM & QM/MM & QM & QM/MM \\
\hline${ }^{2} \mathbf{C}_{R}$ & 0.00 & 0.00 & 0.00 & 0.00 \\
\hline${ }^{4} \mathbf{C}_{R}$ & 10.73 & 12.27 & 9.25 & 10.84 \\
\hline${ }^{6} \mathbf{C}_{R}$ & 14.11 & 13.82 & 11.34 & 11.10 \\
\hline${ }^{2} \mathbf{C}_{\mathrm{Ia}}$ & 23.62 & 23.25 & 20.35 & 20.02 \\
\hline${ }^{2} \mathbf{C}_{\mathrm{Ib}}$ & 20.31 & 20.14 & 16.57 & 16.45 \\
\hline${ }^{2} \mathbf{P}$ & 0.44 & -0.85 & -6.43 & -7.67 \\
\hline${ }^{4} \mathbf{C}_{\mathrm{R}} \mathbf{H}$ & 0.00 & 0.00 & 0.00 & 0.00 \\
\hline${ }^{2} \mathbf{C}_{\mathrm{R}} \mathbf{H}$ & 7.89 & 3.68 & 7.06 & 2.77 \\
\hline${ }^{6} \mathbf{C}_{\mathrm{R}} \mathbf{H}$ & 11.94 & 8.95 & 7.76 & 4.66 \\
\hline${ }^{2} \mathbf{T S} \mathbf{S O O}_{\mathbf{O}} \mathbf{H}$ & 14.32 & 13.62 & 11.66 & 11.02 \\
\hline${ }^{2} \mathbf{C}_{\mathrm{Ia}} \mathbf{H}$ & 6.05 & 5.70 & 0.32 & -0.02 \\
\hline${ }^{2} \mathbf{C}_{\mathrm{Ib}} \mathbf{H}$ & 2.31 & 2.00 & -3.31 & -3.57 \\
\hline${ }^{2} \mathbf{P H}$ & -35.22 & -36.59 & -43.78 & -45.13 \\
\hline ASPP136 ${ }^{2} \mathbf{C}_{\mathrm{R}}^{\mathrm{a}, \mathrm{b}}$ & 23.54 & 17.89 & 16.37 & 15.03 \\
\hline ASPP136 ${ }^{2} \mathbf{P}^{\mathrm{a}, \mathrm{c}}$ & 46.12 & 46.88 & 49.38 & 50.22 \\
\hline
\end{tabular}

${ }^{\mathrm{a}}$ Asp 136 is protonated here. ${ }^{\mathrm{b}}$ Take ${ }^{2} \mathbf{C}_{\mathrm{R}} \mathbf{H}$ as zero point in energy. ${ }^{\mathrm{c}}$ Take ${ }^{2} \mathbf{P H}$ as zero point in energy. 
Table S5. The Calculated Energies (a. u.) from the 116 ps Snapshot

\begin{tabular}{|l|c|c|c|c|}
\hline Energies & \multicolumn{2}{|c|}{ B1 } & \multicolumn{2}{c|}{ B2 } \\
\hline & QM & QM/MM & QM & QM/MM \\
\hline${ }^{2} \mathbf{C}_{\mathrm{R}}$ & -2418.940498 & -2493.862208 & -3559.947478 & -3634.869133 \\
\hline${ }^{4} \mathbf{C}_{\mathrm{R}}$ & -2418.925084 & -2493.845370 & -3559.934503 & -3634.854767 \\
\hline${ }^{6} \mathbf{C}_{\mathrm{R}}$ & -2418.920181 & -2493.844369 & -3559.931522 & -3634.855664 \\
\hline${ }^{2} \mathbf{C}_{\mathrm{Ia}}$ & -2418.905290 & -2493.829029 & -3559.917577 & -3634.841208 \\
\hline${ }^{2} \mathbf{C}_{\mathrm{Ib}}$ & -2418.909374 & -2493.833517 & -3559.922477 & -3634.846526 \\
\hline${ }^{4} \mathbf{C}_{\mathrm{R}} \mathbf{H}$ & -2419.621313 & -2494.556431 & -3560.628361 & -3635.563416 \\
\hline${ }^{2} \mathbf{C}_{\mathrm{R}} \mathbf{H}$ & -2419.617977 & -2494.551743 & -3560.621661 & -3635.555352 \\
\hline${ }^{6} \mathbf{C}_{\mathrm{R}} \mathbf{H}$ & -2419.609107 & -2494.545779 & -3560.618512 & -3635.555073 \\
\hline${ }^{2} \mathbf{T} \mathbf{S}_{\mathbf{O O}} \mathbf{H}$ & -2419.592528 & -2494.527949 & -3560.598562 & -3635.53392 \\
\hline${ }^{2} \mathbf{C}_{\mathrm{Ia}} \mathbf{H}$ & -2419.600898 & -2494.537864 & -3560.610936 & -3635.547872 \\
\hline${ }^{2} \mathbf{C}_{\mathrm{Ib}} \mathbf{H}$ & -2419.606020 & -2494.545738 & -3560.616759 & -3635.556406 \\
\hline
\end{tabular}

Table S6. The Calculated Relative Energies (kcal/mol) from the 116 ps Snapshot

\begin{tabular}{|l|c|c|c|c|}
\hline Energies & \multicolumn{2}{|c|}{$\mathrm{B} 1$} & \multicolumn{2}{c|}{ B2 } \\
\hline & QM & QM/MM & QM & QM/MM \\
\hline${ }^{2} \mathbf{C}_{\mathrm{R}}$ & 0.00 & 0.00 & 0.00 & 0.00 \\
\hline${ }^{4} \mathbf{C}_{\mathrm{R}}$ & 9.67 & 10.57 & 8.14 & 9.02 \\
\hline${ }^{6} \mathbf{C}_{\mathrm{R}}$ & 12.75 & 11.19 & 10.01 & 8.45 \\
\hline${ }^{2} \mathbf{C}_{\mathrm{Ia}}$ & 22.09 & 20.82 & 18.76 & 17.52 \\
\hline${ }^{2} \mathbf{C}_{\mathrm{Ib}}$ & 19.53 & 18.00 & 15.69 & 14.19 \\
\hline${ }^{4} \mathbf{C}_{\mathrm{R}} \mathbf{H}$ & 0.00 & 0.00 & 0.00 & 0.00 \\
\hline${ }^{2} \mathbf{C}_{\mathrm{R}} \mathbf{H}$ & 2.09 & 2.94 & 4.20 & 5.06 \\
\hline${ }^{6} \mathbf{C}_{\mathrm{R}} \mathbf{H}$ & 7.66 & 6.68 & 6.18 & 5.24 \\
\hline${ }^{2} \mathbf{T S}_{\mathbf{O O}} \mathbf{H}$ & 15.97 & 14.93 & 14.49 & 13.45 \\
\hline${ }^{2} \mathbf{C}_{\mathrm{Ia}} \mathbf{H}$ & 10.72 & 8.71 & 6.73 & 4.69 \\
\hline${ }^{2} \mathbf{C}_{\mathrm{Ib}} \mathbf{H}$ & 7.50 & 3.77 & 3.08 & -0.66 \\
\hline
\end{tabular}


Table S7. The Calculated Energies (a. u.) from the 287 ps Snapshot

\begin{tabular}{|l|c|c|c|c|}
\hline Energies & \multicolumn{2}{|c|}{$\mathrm{B} 1$} & \multicolumn{2}{c|}{$\mathrm{B} 2$} \\
\hline & QM & QM/MM & QM & QM/MM \\
\hline${ }^{2} \mathbf{C}_{\mathrm{R}}$ & -2418.951088 & -2493.880425 & -3559.962858 & -3634.892086 \\
\hline${ }^{4} \mathbf{C}_{\mathrm{R}}$ & -2418.935123 & -2493.860273 & -3559.949247 & -3634.874299 \\
\hline${ }^{6} \mathbf{C}_{\mathrm{R}}$ & -2418.931144 & -2493.860358 & -3559.947053 & -3634.876237 \\
\hline${ }^{2} \mathbf{C}_{\mathrm{Ia}}$ & -2418.917072 & -2493.843590 & -3559.934342 & -3634.860843 \\
\hline${ }^{2} \mathbf{C}_{\mathrm{Ib}}$ & -2418.930064 & -2493.854780 & -3559.946409 & -3634.871088 \\
\hline${ }^{4} \mathbf{C}_{\mathrm{R}} \mathbf{H}$ & -2419.637644 & -2494.573678 & -3560.650712 & -3635.586619 \\
\hline${ }^{2} \mathbf{C}_{\mathrm{R}} \mathbf{H}$ & -2419.631204 & -2494.56959 & -3560.640855 & -3635.579135 \\
\hline${ }^{6} \mathbf{C}_{\mathrm{R}} \mathbf{H}$ & -2419.625475 & -2494.563263 & -3560.64054 & -3635.578229 \\
\hline${ }^{2} \mathbf{T S}_{\mathbf{O O}} \mathbf{H}$ & -2419.601911 & -2494.541754 & -3560.618817 & -3635.558551 \\
\hline${ }^{2} \mathbf{C}_{\mathrm{Ia}} \mathbf{H}$ & -2419.612085 & -2494.553478 & -3560.631066 & -3635.572404 \\
\hline${ }^{2} \mathbf{C}_{\mathrm{Ib}} \mathbf{H}$ & -2419.617815 & -2494.558599 & -3560.637226 & -3635.577938 \\
\hline
\end{tabular}

Table S8. The Calculated Relative Energies (kcal/mol) from the 287 ps Snapshot

\begin{tabular}{|l|c|c|c|c|}
\hline Energies & \multicolumn{2}{|c|}{$\mathrm{B} 1$} & \multicolumn{2}{c|}{ B2 } \\
\hline & QM & QM/MM & QM & QM/MM \\
\hline${ }^{2} \mathbf{C}_{\mathrm{R}}$ & 0.00 & 0.00 & 0.00 & 0.00 \\
\hline${ }^{4} \mathbf{C}_{\mathrm{R}}$ & 10.02 & 12.65 & 8.54 & 11.16 \\
\hline${ }^{6} \mathbf{C}_{\mathrm{R}}$ & 12.52 & 12.59 & 9.92 & 9.95 \\
\hline${ }^{2} \mathbf{C}_{\mathrm{Ia}}$ & 21.35 & 23.11 & 17.89 & 19.61 \\
\hline${ }^{2} \mathbf{C}_{\mathrm{Ib}}$ & 13.19 & 16.09 & 10.32 & 13.18 \\
\hline${ }^{4} \mathbf{C}_{\mathrm{R}} \mathbf{H}$ & 0.00 & 0.00 & 0.00 & 0.00 \\
\hline${ }^{2} \mathbf{C}_{\mathrm{R}} \mathbf{H}$ & 4.04 & 2.57 & 6.19 & 4.70 \\
\hline${ }^{6} \mathbf{C}_{\mathrm{R}} \mathbf{H}$ & 7.64 & 6.54 & 6.38 & 5.26 \\
\hline${ }^{2} \mathbf{T S}_{\mathbf{O O}} \mathbf{H}$ & 18.38 & 17.47 & 13.83 & 12.92 \\
\hline${ }^{2} \mathbf{C}_{\mathrm{Ia}} \mathbf{H}$ & 12.00 & 10.11 & 6.14 & 4.22 \\
\hline${ }^{2} \mathbf{C}_{\mathrm{Ib}} \mathbf{H}$ & 8.40 & 6.90 & 2.28 & 0.75 \\
\hline
\end{tabular}




\section{Spin and charge distribution}

Table S9. The Calculated Spin Distribution and Expectation Value $<\mathrm{S}^{2}>$ from the 0 ps Snapshot at the QM/MM B1 Level.

\begin{tabular}{|l|c|c|c|c|c|c|c|c|}
\hline & Fe & O proximal & OH distal & ImH(His20) & Porp & Asp136 & Arg132 & $<\mathrm{S}^{2}>$ \\
\hline${ }^{2} \mathbf{C}_{\mathrm{R}}$ & 0.9551 & 0.1140 & 0.0029 & -0.0171 & -0.0551 & 0.0000 & 0.0000 & 0.7756 \\
\hline${ }^{4} \mathbf{C}_{\mathrm{R}}$ & 2.7317 & 0.2388 & 0.0318 & 0.0709 & -0.0731 & 0.0000 & 0.0000 & 3.8010 \\
\hline${ }^{6} \mathbf{C}_{\mathrm{R}}$ & 4.0941 & 0.3062 & 0.0411 & 0.0786 & 0.4796 & 0.0000 & 0.0000 & 8.7625 \\
\hline${ }^{2} \mathbf{C}_{\mathrm{Ia}}$ & 1.3193 & 0.6746 & -0.8583 & -0.0179 & -0.1236 & 0.0000 & 0.0000 & 1.6845 \\
\hline${ }^{2} \mathbf{C}_{\mathrm{Ib}}$ & 1.3916 & 0.6980 & -0.8746 & -0.0155 & -0.2026 & 0.0000 & 0.0000 & 1.7811 \\
\hline${ }^{2} \mathbf{P}$ & 1.3651 & 0.7250 & -0.0152 & -0.0236 & -1.0502 & 0.0000 & 0.0000 & 1.7990 \\
\hline ASPP136 ${ }^{2} \mathbf{C}_{\mathrm{R}}{ }^{\mathrm{a}}$ & 0.9709 & 0.1003 & 0.0012 & -0.0169 & -0.0554 & 0.0000 & 0.0000 & 0.7731 \\
\hline ASPP136 ${ }^{2} \mathbf{P}^{\mathrm{a}}$ & 1.3951 & 0.7006 & -0.0153 & -0.0251 & -1.0546 & 0.0000 & -0.0001 & 1.7999 \\
\hline & $\mathrm{Fe}$ & OH proximal & OH distal & ImH(His20) & Porp & Asp136 & Arg132 & \\
\hline${ }^{2} \mathbf{C}_{\mathrm{R}} \mathbf{H}$ & 1.0648 & -0.0027 & -0.0022 & -0.0067 & -0.0532 & 0.0000 & 0.0000 & 0.8235 \\
\hline${ }^{4} \mathbf{C}_{\mathrm{R}} \mathbf{H}$ & 2.7979 & -0.0075 & 0.0010 & 0.1566 & 0.0520 & 0.0000 & 0.0000 & 3.8031 \\
\hline${ }^{6} \mathbf{C}_{\mathrm{R}} \mathbf{H}$ & 4.1257 & 0.0100 & -0.0070 & 0.1648 & 0.7060 & 0.0000 & 0.0000 & 8.7580 \\
\hline${ }^{2} \mathbf{T} \mathbf{S}_{\mathbf{O o}} \mathbf{H}$ & 0.9462 & -0.0824 & -0.3466 & 0.0101 & 0.4733 & 0.0000 & 0.0000 & 1.1671 \\
\hline${ }^{2} \mathbf{C}_{\mathrm{Ia}} \mathbf{H}$ & 0.9711 & -0.1756 & -0.7346 & 0.0078 & 0.9304 & 0.0001 & 0.0000 & 1.7167 \\
\hline${ }^{2} \mathbf{C}_{\mathrm{Ib}} \mathbf{H}$ & 0.9852 & 0.0602 & -0.9075 & 0.0081 & 0.8514 & 0.0001 & 0.0000 & 1.7094 \\
\hline${ }^{2} \mathbf{P H}$ & 1.0134 & 0.0457 & 0.0021 & -0.0024 & -0.0587 & 0.0000 & 0.0000 & 0.7759 \\
\hline
\end{tabular}

${ }^{\mathrm{a}}$ Asp136 is protonated here.

Table S10. The Calculated Mulliken Charge Distribution from the 0 ps Snapshot at the QM/MM B1 Level.

\begin{tabular}{|l|c|c|c|c|c|c|c|}
\hline & Fe & O proximal & OH distal & ImH(His20) & Porp & Asp136 & Arg132 \\
\hline${ }^{2} \mathbf{C}_{\mathrm{R}}$ & 0.5827 & -0.2536 & -0.1443 & 0.2719 & -0.5039 & -0.7188 & 0.7178 \\
\hline${ }^{4} \mathbf{C}_{\mathrm{R}}$ & 0.6733 & -0.3432 & -0.1424 & 0.2219 & -0.4531 & -0.7216 & 0.7190 \\
\hline${ }^{6} \mathbf{C}_{\mathrm{R}}$ & 0.8281 & -0.3312 & -0.1075 & 0.2410 & -0.6768 & -0.7210 & 0.7184 \\
\hline${ }^{2} \mathbf{C}_{\mathrm{Ia}}$ & 0.6115 & -0.4008 & -0.1629 & 0.2957 & -0.3654 & -0.7234 & 0.7160 \\
\hline${ }^{2} \mathbf{C}_{\mathrm{Ib}}$ & 0.6662 & -0.5288 & -0.1568 & 0.2999 & -0.2966 & -0.7234 & 0.7132 \\
\hline${ }^{2} \mathbf{P}$ & 0.6707 & -0.5038 & -0.3630 & 0.2858 & -0.1340 & -0.7272 & 0.7242 \\
\hline ASPP136 ${ }^{2} \mathbf{C}_{\mathrm{R}}{ }^{\mathrm{a}}$ & 0.5907 & -0.2519 & -0.1718 & 0.2951 & -0.4684 & -0.0110 & 0.7540 \\
\hline ASPP136 ${ }^{2} \mathbf{P}^{\mathrm{a}}$ & 0.6821 & -0.5228 & -0.3737 & 0.3063 & -0.0886 & -0.0616 & 0.7771 \\
\hline & $\mathrm{Fe}$ & $\mathrm{OH}$ proximal & OH distal & ImH(His20) & Porp & Asp136 & Arg132 \\
\hline${ }^{2} \mathbf{C}_{\mathrm{R}} \mathbf{H}$ & 0.5600 & 0.2939 & -0.1342 & 0.3837 & -0.1800 & -0.7120 & 0.7222 \\
\hline${ }^{4} \mathbf{C}_{\mathrm{R}} \mathbf{H}$ & 0.8513 & -0.0265 & -0.1292 & 0.2980 & -0.1236 & -0.7006 & 0.7252 \\
\hline${ }^{6} \mathbf{C}_{\mathrm{R}} \mathbf{H}$ & 0.8647 & 0.2340 & -0.1678 & 0.3080 & -0.3150 & -0.7136 & 0.7226 \\
\hline${ }^{2} \mathbf{T} \mathbf{S}_{\mathbf{O o}} \mathbf{H}$ & 0.5662 & 0.1418 & -0.2378 & 0.3611 & 0.1055 & -0.7172 & 0.7244 \\
\hline${ }^{2} \mathbf{C}_{\mathrm{Ia}} \mathbf{H}$ & 0.6179 & -0.1124 & -0.2852 & 0.3356 & 0.3948 & -0.7209 & 0.7256 \\
\hline${ }^{2} \mathbf{C}_{\mathrm{Ib}} \mathbf{H}$ & 0.6608 & -0.3768 & -0.0951 & 0.3293 & 0.4498 & -0.7205 & 0.7212 \\
\hline${ }^{2} \mathbf{P H}$ & 0.6672 & -0.3615 & -0.3057 & 0.3202 & 0.6143 & -0.7240 & 0.7314 \\
\hline
\end{tabular}

${ }^{\mathrm{a}}$ Asp136 is protonated here. 
Table S11. The Calculated Spin Distribution and Expectation Value $<\mathrm{S}^{2}>$ from the 0 ps Snapshot at the QM/MM B2 Level.

\begin{tabular}{|l|c|c|c|c|c|c|c|c|}
\hline & Fe & O proximal & OH distal & ImH(His20) & Porp & Asp136 & Arg132 & $<\mathrm{S}^{2}>$ \\
\hline${ }^{2} \mathbf{C}_{\mathrm{R}}$ & 0.9527 & 0.1003 & 0.0037 & -0.0086 & -0.0485 & 0.0000 & 0.0000 & 0.7693 \\
\hline${ }^{4} \mathbf{C}_{\mathrm{R}}$ & 2.7639 & 0.1993 & 0.0335 & 0.0846 & -0.0820 & 0.0000 & -0.0001 & 3.7929 \\
\hline${ }^{6} \mathbf{C}_{\mathrm{R}}$ & 4.2087 & 0.2462 & 0.0399 & 0.0906 & 0.4138 & 0.0000 & -0.0001 & 8.7615 \\
\hline${ }^{2} \mathbf{C}_{\mathrm{Ia}}$ & 1.3749 & 0.5891 & -0.8407 & 0.0027 & -0.1293 & 0.0000 & 0.0000 & 1.6680 \\
\hline${ }^{2} \mathbf{C}_{\mathrm{Ib}}$ & 1.4245 & 0.6416 & -0.8609 & 0.0049 & -0.2107 & 0.0000 & -0.0003 & 1.7799 \\
\hline${ }^{2} \mathbf{P}$ & 1.4247 & 0.6666 & -0.0239 & -0.0076 & -1.0573 & 0.0000 & -0.0002 & 1.8019 \\
\hline ASPP136 ${ }^{2} \mathbf{C}_{\mathrm{R}}{ }^{\mathrm{a}}$ & 0.9646 & 0.0872 & 0.0028 & -0.0098 & -0.0453 & 0.0000 & -0.0002 & 0.7678 \\
\hline ASPP136 ${ }^{2} \mathbf{P}^{\mathrm{a}}$ & 1.4482 & 0.6419 & -0.0233 & -0.0083 & -1.0552 & 0.0000 & 0.0000 & 1.8011 \\
\hline & $\mathrm{Fe}$ & OH proximal & OH distal & ImH(His20) & Porp & Asp136 & Arg132 & \\
\hline${ }^{2} \mathbf{C}_{\mathrm{R}} \mathbf{H}$ & 1.0428 & -0.0065 & 0.0017 & -0.0048 & -0.0334 & 0.0000 & 0.0000 & 0.8080 \\
\hline${ }^{4} \mathbf{C}_{\mathrm{R}} \mathbf{H}$ & 2.8013 & 0.0040 & -0.0009 & 0.1413 & 0.0534 & 0.0001 & 0.0000 & 3.7951 \\
\hline${ }^{6} \mathbf{C}_{\mathrm{R}} \mathbf{H}$ & 4.1729 & 0.0196 & 0.0029 & 0.1481 & 0.6564 & 0.0000 & 0.0000 & 8.7581 \\
\hline${ }^{2} \mathbf{T} \mathbf{S}_{\mathbf{O o O}} \mathbf{H}$ & 0.9134 & -0.0877 & -0.3484 & 0.0172 & 0.5058 & -0.0001 & 0.0001 & 1.1661 \\
\hline${ }^{2} \mathbf{C}_{\mathrm{Ia}} \mathbf{H}$ & 0.9384 & -0.1849 & -0.7306 & 0.0175 & 0.9594 & 0.0000 & 0.0000 & 1.7053 \\
\hline${ }^{2} \mathbf{C}_{\mathrm{Ib}} \mathbf{H}$ & 0.9702 & 0.0448 & -0.8880 & 0.0181 & 0.8529 & 0.0001 & -0.0001 & 1.6868 \\
\hline${ }^{2} \mathbf{P} \mathbf{H}$ & 1.0039 & 0.0337 & 0.0018 & 0.0064 & -0.0461 & 0.0000 & 0.0000 & 0.7714 \\
\hline
\end{tabular}

${ }^{\mathrm{a}}$ Asp136 is protonated here.

Table S12. The Calculated Mulliken Charge Distribution from the 0 ps Snapshot at the QM/MM B2 Level.

\begin{tabular}{|l|c|c|c|c|c|c|c|}
\hline & $\mathrm{Fe}$ & O proximal & OH distal & ImH(His20) & Porp & Asp136 & Arg132 \\
\hline${ }^{2} \mathbf{C}_{\mathrm{R}}$ & 0.8367 & -0.0103 & -0.0479 & 0.5250 & -1.2199 & -0.9028 & 0.8924 \\
\hline${ }^{4} \mathbf{C}_{\mathrm{R}}$ & 1.1459 & -0.2759 & -0.0843 & 0.2422 & -0.9400 & -0.9034 & 0.8929 \\
\hline${ }^{6} \mathbf{C}_{\mathrm{R}}$ & 1.1511 & -0.1766 & -0.0665 & 0.3196 & -1.1424 & -0.9046 & 0.8929 \\
\hline${ }^{2} \mathbf{C}_{\mathrm{Ia}}$ & 0.9977 & 0.0047 & -0.0759 & 0.2431 & -1.0687 & -0.9084 & 0.8904 \\
\hline${ }^{2} \mathbf{C}_{\mathrm{Ib}}$ & 1.0097 & -0.1218 & -0.0963 & 0.2539 & -0.9325 & -0.9104 & 0.8923 \\
\hline${ }^{2} \mathbf{P}$ & 1.2153 & -0.1700 & -0.2269 & 0.1084 & -0.8305 & -0.9092 & 0.8926 \\
\hline ASPP136 ${ }^{2} \mathbf{C}_{\mathrm{R}}{ }^{\mathrm{a}}$ & 0.8982 & -0.0272 & -0.0806 & 0.5814 & -1.2385 & -0.0300 & 0.8648 \\
\hline $\mathrm{ASPP} 136{ }^{2} \mathbf{P}^{\mathrm{a}}$ & 1.1986 & -0.1741 & -0.2463 & 0.1237 & -0.7688 & -0.0362 & 0.8426 \\
\hline & $\mathrm{Fe}$ & $\mathrm{OH}$ proximal & OH distal & ImH(His20) & Porp & Asp136 & Arg 132 \\
\hline${ }^{2} \mathbf{C}_{\mathrm{R}} \mathbf{H}$ & 0.8712 & 0.5301 & -0.1589 & 1.0122 & -1.2032 & -0.9152 & 0.9042 \\
\hline${ }^{4} \mathbf{C}_{\mathrm{R}} \mathbf{H}$ & 1.0537 & 0.0780 & -0.0365 & 0.3653 & -0.3816 & -0.8947 & 0.8922 \\
\hline${ }^{6} \mathbf{C}_{\mathrm{R}} \mathbf{H}$ & 1.2363 & 0.3316 & -0.1471 & 0.6723 & -1.0266 & -0.9160 & 0.9014 \\
\hline${ }^{2} \mathbf{T} \mathbf{S}_{\mathbf{O O}} \mathbf{H}$ & 0.6232 & 0.4355 & -0.2010 & 0.9288 & -0.7282 & -0.9155 & 0.9011 \\
\hline${ }^{2} \mathbf{C}_{\mathrm{Ia}} \mathbf{H}$ & 0.7520 & 0.1271 & -0.2096 & 0.6977 & -0.2934 & -0.9148 & 0.8984 \\
\hline${ }^{2} \mathbf{C}_{\mathrm{Ib}} \mathbf{H}$ & 0.7144 & -0.1812 & -0.0654 & 0.7003 & -0.0809 & -0.9187 & 0.8987 \\
\hline${ }^{2} \mathbf{P H}$ & 0.9029 & -0.2039 & -0.1780 & 0.5568 & -0.0093 & -0.9204 & 0.8998 \\
\hline
\end{tabular}

${ }^{\mathrm{a}}$ Asp136 is protonated here. 
Table S13. The Calculated Spin Distribution and Expectation Value $<\mathrm{S}^{2}>$ from the 116 ps Snapshot at the QM/MM B1 Level.

\begin{tabular}{|c|c|c|c|c|c|c|c|c|}
\hline & $\mathrm{Fe}$ & O proximal & OH distal & $\operatorname{ImH}($ His 20$)$ & Porp & Asp136 & $\operatorname{Arg} 132$ & $\left.<\mathrm{S}^{2}\right\rangle$ \\
\hline${ }^{2} \mathbf{C}_{\mathrm{R}}$ & 0.8917 & 0.1564 & 0.0114 & -0.0115 & -0.0477 & 0.0000 & 0.0000 & 0.7697 \\
\hline${ }^{4} \mathbf{C}_{\mathrm{R}}$ & 2.7032 & 0.2743 & 0.0395 & 0.0530 & -0.0706 & 0.0000 & 0.0000 & 3.8020 \\
\hline${ }^{6} \mathbf{C}_{\mathrm{R}}$ & 4.0709 & 0.3407 & 0.0511 & 0.0607 & 0.4759 & 0.0000 & 0.0000 & 8.7643 \\
\hline${ }^{2} \mathbf{C}_{\mathrm{Ia}}$ & 1.2971 & 0.6657 & -0.8677 & -0.0159 & -0.0864 & 0.0000 & 0.0000 & 1.6701 \\
\hline \multirow[t]{2}{*}{${ }^{2} \mathbf{C}_{\mathrm{Ib}}$} & 1.3571 & 0.7222 & -0.8946 & -0.0128 & -0.1771 & 0.0001 & 0.0000 & 1.7771 \\
\hline & $\mathrm{Fe}$ & OH proximal & OH distal & $\operatorname{ImH}($ His 20$)$ & Porp & Asp136 & $\operatorname{Arg} 132$ & \\
\hline${ }^{2} \mathbf{C}_{\mathrm{R}} \mathbf{H}$ & 1.0568 & -0.0081 & -0.0001 & -0.0151 & -0.0338 & 0.0000 & 0.0000 & 0.8424 \\
\hline${ }^{4} \mathbf{C}_{\mathrm{R}} \mathbf{H}$ & 2.8614 & -0.0067 & 0.0026 & 0.1251 & 0.0172 & 0.0000 & 0.0000 & 3.8052 \\
\hline${ }^{6} \mathbf{C}_{\mathrm{R}} \mathbf{H}$ & 4.1315 & -0.0039 & -0.0010 & 0.1372 & 0.7361 & 0.0000 & 0.0000 & 8.7586 \\
\hline${ }^{2} \mathrm{TS}_{\mathrm{OO}} \mathrm{H}$ & 0.9000 & -0.0802 & -0.3923 & 0.0041 & 0.5738 & -0.0002 & 0.0000 & 1.2584 \\
\hline${ }^{2} \mathbf{C}_{\mathrm{Ia}} \mathbf{H}$ & 0.9130 & -0.1365 & -0.7448 & 0.0087 & 0.9595 & -0.0004 & 0.0001 & 1.7214 \\
\hline${ }^{2} \mathbf{C}_{\mathrm{Ib}} \mathbf{H}$ & 0.9543 & 0.0794 & -0.9868 & 0.0040 & 0.9479 & -0.0001 & 0.0000 & 1.7704 \\
\hline
\end{tabular}

Table S14. The Calculated Mulliken Charge Distribution from the 116 ps Snapshot at the QM/MM B1 Level.

\begin{tabular}{|l|c|c|c|c|c|c|c|}
\hline & Fe & O proximal & OH distal & ImH(His20) & Porp & Asp136 & Arg132 \\
\hline${ }^{2} \mathbf{C}_{\mathrm{R}}$ & 0.5497 & -0.1356 & -0.2126 & 0.2547 & -0.4915 & -0.6336 & 0.6108 \\
\hline${ }^{4} \mathbf{C}_{\mathrm{R}}$ & 0.6532 & -0.2275 & -0.2073 & 0.1950 & -0.4418 & -0.6360 & 0.6108 \\
\hline${ }^{6} \mathbf{C}_{\mathrm{R}}$ & 0.8049 & -0.2203 & -0.1699 & 0.2135 & -0.6603 & -0.6360 & 0.6130 \\
\hline${ }^{2} \mathbf{C}_{\mathrm{Ia}}$ & 0.5841 & -0.3249 & -0.1619 & 0.2649 & -0.3668 & -0.6372 & 0.6060 \\
\hline${ }^{2} \mathbf{C}_{\mathrm{Ib}}$ & 0.6365 & -0.4590 & -0.1448 & 0.2672 & -0.2937 & -0.6373 & 0.6060 \\
\hline & $\mathrm{Fe}$ & OH proximal & OH distal & ImH(His20) & Porp & Asp136 & Arg132 \\
\hline & 0.5846 & 0.2945 & -0.1207 & 0.3427 & -0.1694 & -0.6452 & 0.6558 \\
\hline${ }^{2} \mathbf{C}_{\mathrm{R}} \mathbf{H}$ & 0.7096 & 0.2061 & -0.1174 & 0.2621 & -0.1236 & -0.6468 & 0.6542 \\
\hline${ }^{4} \mathbf{C}_{\mathrm{R}} \mathbf{H}$ & 0.8553 & 0.2249 & -0.1276 & 0.2768 & -0.2915 & -0.6470 & 0.6540 \\
\hline${ }^{6} \mathbf{C}_{\mathrm{R}} \mathbf{H}$ & 0.5848 & 0.1490 & -0.2757 & 0.3213 & 0.1570 & -0.6460 & 0.6496 \\
\hline${ }^{2} \mathbf{T} \mathbf{S}_{\mathbf{O o}} \mathbf{H}$ & 0.6746 & 0.1123 & -0.1600 & 0.4103 & 0.0501 & -0.8346 & 0.8441 \\
\hline${ }^{2} \mathbf{C}_{\mathrm{II}} \mathbf{H}$ & 0.6701 & -0.3436 & -0.0584 & 0.3014 & 0.4011 & -0.6489 & 0.6480 \\
\hline${ }^{2} \mathbf{C}_{\mathrm{Ib}} \mathbf{H}$ & & & & & & \\
\hline
\end{tabular}


Table S15. The Calculated Spin Distribution and Expectation Value $<\mathrm{S}^{2}>$ from the 116 ps Snapshot at the QM/MM B2 Level.

\begin{tabular}{|c|c|c|c|c|c|c|c|c|}
\hline & $\mathrm{Fe}$ & O proximal & OH distal & $\operatorname{ImH}($ His20) & Porp & Asp136 & $\operatorname{Arg} 132$ & $\left.<\mathrm{S}^{2}\right\rangle$ \\
\hline${ }^{2} \mathbf{C}_{\mathrm{R}}$ & 0.8975 & 0.1368 & 0.0123 & -0.0034 & -0.0431 & 0.0000 & 0.0001 & 0.7659 \\
\hline${ }^{4} \mathbf{C}_{\mathrm{R}}$ & 2.7344 & 0.2362 & 0.0385 & 0.0670 & -0.0775 & 0.0000 & 0.0000 & 3.7942 \\
\hline${ }^{6} \mathbf{C}_{\mathrm{R}}$ & 4.1816 & 0.2789 & 0.0470 & 0.0756 & 0.4157 & 0.0001 & 0.0000 & 8.7634 \\
\hline${ }^{2} \mathbf{C}_{\mathrm{Ia}}$ & 1.3381 & 0.6012 & -0.8457 & 0.0028 & -0.1012 & 0.0000 & 0.0001 & 1.6564 \\
\hline${ }^{2} \mathbf{C}_{\mathrm{Ib}}$ & 1.3839 & 0.6763 & -0.8827 & 0.0065 & -0.1873 & 0.0000 & 0.0000 & 1.7782 \\
\hline & $\mathrm{Fe}$ & OH proximal & $\mathrm{OH}$ distal & $\operatorname{ImH}($ His20) & Porp & Asp136 & Arg132 & \\
\hline${ }^{2} \mathbf{C}_{\mathrm{R}} \mathbf{H}$ & 1.0344 & -0.0029 & 0.0007 & -0.0107 & -0.0217 & 0.0000 & 0.0000 & 0.8240 \\
\hline${ }^{4} \mathbf{C}_{\mathrm{R}} \mathbf{H}$ & 2.8331 & 0.0135 & 0.0042 & 0.1220 & 0.0266 & 0.0000 & 0.0000 & 3.7962 \\
\hline${ }^{6} \mathbf{C}_{\mathrm{R}} \mathbf{H}$ & 4.1751 & 0.0194 & 0.0008 & 0.1278 & 0.6768 & 0.0000 & 0.0001 & 8.7586 \\
\hline${ }^{2} \mathrm{TS}_{\mathrm{OO}} \mathrm{H}$ & 0.8596 & -0.0875 & -0.4009 & 0.0180 & 0.6152 & -0.0003 & 0.0000 & 1.2662 \\
\hline${ }^{2} \mathbf{C}_{\mathrm{Ia}} \mathbf{H}$ & 0.9331 & -0.1210 & -0.7529 & 0.0001 & 0.9391 & -0.0004 & 0.0000 & 1.7157 \\
\hline${ }^{2} \mathbf{C}_{\mathrm{Ib}} \mathrm{H}$ & 0.9505 & 0.0632 & -0.9889 & 0.0107 & 0.9598 & -0.0001 & -0.0002 & 1.7663 \\
\hline
\end{tabular}

Table S16. The Calculated Mulliken Charge Distribution from the 116 ps Snapshot at the QM/MM B2 Level.

\begin{tabular}{|l|c|c|c|c|c|c|c|}
\hline & Fe & O proximal & OH distal & ImH(His20) & Porp & Asp136 & Arg132 \\
\hline${ }^{2} \mathbf{C}_{\mathrm{R}}$ & 0.9337 & 0.1292 & -0.1153 & 0.3420 & -1.1873 & -0.9022 & 0.8535 \\
\hline${ }^{4} \mathbf{C}_{\mathrm{R}}$ & 1.2350 & -0.1490 & -0.1367 & 0.0632 & -0.8995 & -0.8988 & 0.8498 \\
\hline${ }^{6} \mathbf{C}_{\mathrm{R}}$ & 1.2548 & -0.0539 & -0.1194 & 0.1168 & -1.0893 & -0.8997 & 0.8524 \\
\hline${ }^{2} \mathbf{C}_{\mathrm{Ia}}$ & 1.0753 & 0.0664 & -0.0581 & 0.0702 & -1.0360 & -0.9016 & 0.8447 \\
\hline${ }^{2} \mathbf{C}_{\mathrm{Ib}}$ & 1.0727 & -0.0281 & -0.0687 & 0.0559 & -0.9049 & -0.9014 & 0.8430 \\
\hline & $\mathrm{Fe}$ & OH proximal & OH distal & ImH(His20) & Porp & Asp136 & Arg132 \\
\hline & 0.7808 & 0.2147 & -0.0041 & 0.8557 & -0.7839 & -0.8310 & 0.8486 \\
\hline${ }^{2} \mathbf{C}_{\mathrm{R}} \mathbf{H}$ & 1.1739 & 0.0887 & 0.0102 & 0.3568 & -0.5600 & -0.8336 & 0.8498 \\
\hline${ }^{4} \mathbf{C}_{\mathrm{R}} \mathbf{H}$ & 1.2097 & 0.0836 & -0.0008 & 0.5148 & -0.7358 & -0.8344 & 0.8491 \\
\hline${ }^{6} \mathbf{C}_{\mathrm{R}} \mathbf{H}$ & 0.5600 & 0.2857 & -0.1589 & 0.6326 & -0.2446 & -0.8315 & 0.8460 \\
\hline${ }^{2} \mathbf{T} \mathbf{S}_{\mathbf{O o}} \mathbf{H}$ & 0.6243 & -0.1066 & -0.2599 & 0.3027 & 0.3949 & -0.6482 & 0.6452 \\
\hline${ }^{2} \mathbf{C}_{\mathrm{II}} \mathbf{H}$ & 0.6981 & -0.1360 & 0.0261 & 0.3933 & 0.1160 & -0.8363 & 0.8434 \\
\hline${ }^{2} \mathbf{C}_{\mathrm{Ib}} \mathbf{H}$ & & & & & & \\
\hline
\end{tabular}


Table S17. The Calculated Spin Distribution and Expectation Value $<\mathrm{S}^{2}>$ from the 287 ps Snapshot at the QM/MM B1 Level.

\begin{tabular}{|l|c|c|c|c|c|c|c|c|}
\hline & $\mathrm{Fe}$ & O proximal & OH distal & ImH(His20) & Porp & Asp136 & $\operatorname{Arg} 132$ & $\left.<\mathrm{S}^{2}\right\rangle$ \\
\hline${ }^{2} \mathbf{C}_{\mathrm{R}}$ & 0.8974 & 0.1556 & 0.0110 & -0.0138 & -0.0507 & 0.0000 & 0.0000 & 0.7720 \\
\hline${ }^{4} \mathbf{C}_{\mathrm{R}}$ & 2.6856 & 0.2833 & 0.0410 & 0.0598 & -0.0701 & 0.0000 & 0.0000 & 3.8009 \\
\hline${ }^{6} \mathbf{C}_{\mathrm{R}}$ & 4.0661 & 0.3480 & 0.0524 & 0.0636 & 0.4692 & 0.0001 & 0.0000 & 8.7646 \\
\hline${ }^{2} \mathbf{C}_{\mathrm{Ia}}$ & 1.2748 & 0.6837 & -0.8545 & -0.0159 & -0.0930 & 0.0000 & 0.0000 & 1.6602 \\
\hline${ }^{2} \mathbf{C}_{\mathrm{Ib}}$ & 1.3618 & 0.7087 & -0.8719 & -0.0122 & -0.1898 & 0.0000 & 0.0000 & 1.7651 \\
\hline & $\mathrm{Fe}$ & OH proximal & OH distal & ImH(His20) & Porp & Asp136 & Arg132 & \\
\hline${ }^{2} \mathbf{C}_{\mathrm{R}} \mathbf{H}$ & 1.0695 & -0.0065 & -0.0022 & -0.0171 & -0.0438 & 0.0000 & 0.0000 & 0.8454 \\
\hline${ }^{4} \mathbf{C}_{\mathrm{R}} \mathbf{H}$ & 2.8583 & -0.0064 & 0.0010 & 0.1266 & 0.0201 & 0.0001 & 0.0000 & 3.8054 \\
\hline${ }^{6} \mathbf{C}_{\mathrm{R}} \mathbf{H}$ & 4.1286 & -0.0023 & -0.0028 & 0.1388 & 0.7378 & 0.0000 & 0.0000 & 8.7587 \\
\hline${ }^{2} \mathbf{T S} \mathbf{S}_{\mathbf{O o}} \mathbf{H}$ & 0.8423 & -0.1277 & -0.4483 & 0.0063 & 0.7382 & -0.0004 & 0.0000 & 1.3679 \\
\hline${ }^{2} \mathbf{C}_{\mathrm{Ia}} \mathbf{H}$ & 0.9520 & -0.1532 & -0.7358 & 0.0050 & 0.9407 & -0.0006 & 0.0000 & 1.7198 \\
\hline${ }^{2} \mathbf{C}_{\mathrm{Ib}} \mathbf{H}$ & 0.9530 & 0.0784 & -0.9892 & 0.0059 & 0.9496 & 0.0000 & 0.0000 & 1.7813 \\
\hline
\end{tabular}

Table S18. The Calculated Mulliken Charge Distribution from the $287 \mathrm{ps}$ Snapshot at the QM/MM B1 Level.

\begin{tabular}{|l|c|c|c|c|c|c|c|}
\hline & $\mathrm{Fe}$ & O proximal & OH distal & ImH(His20) & Porp & Asp136 & Arg132 \\
\hline${ }^{2} \mathbf{C}_{\mathrm{R}}$ & 0.5662 & -0.1590 & -0.1918 & 0.2486 & -0.4783 & -0.6366 & 0.6662 \\
\hline${ }^{4} \mathbf{C}_{\mathrm{R}}$ & 0.6648 & -0.2469 & -0.1844 & 0.1984 & -0.4383 & -0.6372 & 0.6646 \\
\hline${ }^{6} \mathbf{C}_{\mathrm{R}}$ & 0.8163 & -0.2422 & -0.1446 & 0.2136 & -0.6516 & -0.6369 & 0.6658 \\
\hline${ }^{2} \mathbf{C}_{\mathrm{Ia}}$ & 0.5892 & -0.3169 & -0.1819 & 0.2669 & -0.3456 & -0.6356 & 0.6612 \\
\hline${ }^{2} \mathbf{C}_{\mathrm{Ib}}$ & 0.6496 & -0.4685 & -0.2169 & 0.2694 & -0.2478 & -0.6348 & 0.6600 \\
\hline & $\mathrm{Fe}$ & OH proximal & OH distal & ImH(His20) & Porp & Asp136 & Arg132 \\
\hline${ }^{2} \mathbf{C}_{\mathrm{R}} \mathbf{H}$ & 0.6105 & 0.2635 & -0.1434 & 0.3377 & -0.1474 & -0.6378 & 0.6866 \\
\hline${ }^{4} \mathbf{C}_{\mathrm{R}} \mathbf{H}$ & 0.7377 & 0.1930 & -0.1804 & 0.2534 & -0.0941 & -0.6409 & 0.6894 \\
\hline${ }^{6} \mathbf{C}_{\mathrm{R}} \mathbf{H}$ & 0.8908 & 0.2035 & -0.1841 & 0.2698 & -0.2660 & -0.6412 & 0.6880 \\
\hline${ }^{2} \mathbf{T} \mathbf{S}_{\mathbf{O o}} \mathbf{H}$ & 0.5961 & 0.0271 & -0.2263 & 0.3037 & 0.2556 & -0.6442 & 0.6854 \\
\hline${ }^{2} \mathbf{C}_{\mathrm{II}} \mathbf{H}$ & 0.6330 & -0.1320 & -0.2488 & 0.2974 & 0.4175 & -0.6454 & 0.6836 \\
\hline${ }^{2} \mathbf{C}_{\mathrm{Ib}} \mathbf{H}$ & 0.6832 & -0.3424 & -0.0636 & 0.2919 & 0.4210 & -0.6440 & 0.6816 \\
\hline
\end{tabular}


Table S19. The Calculated Spin Distribution and Expectation Value $<\mathrm{S}^{2}>$ from the 287 ps Snapshot at the QM/MM B2 Level.

\begin{tabular}{|c|c|c|c|c|c|c|c|c|}
\hline & $\mathrm{Fe}$ & O proximal & OH distal & $\operatorname{ImH}($ His 20$)$ & Porp & Asp136 & $\operatorname{Arg} 132$ & $<\mathrm{S}^{2}>$ \\
\hline${ }^{2} \mathbf{C}_{\mathrm{R}}$ & 0.9066 & 0.1345 & 0.0116 & -0.0059 & -0.0471 & 0.0000 & 0.0001 & 0.7661 \\
\hline${ }^{4} \mathbf{C}_{\mathrm{R}}$ & 2.7166 & 0.2395 & 0.0401 & 0.0751 & -0.0717 & 0.0001 & 0.0001 & 3.7934 \\
\hline${ }^{6} \mathbf{C}_{\mathrm{R}}$ & 4.1770 & 0.2820 & 0.0481 & 0.0808 & 0.4117 & -0.0001 & 0.0000 & 8.7636 \\
\hline${ }^{2} \mathbf{C}_{\text {Ia }}$ & 1.3354 & 0.6022 & -0.8359 & 0.0036 & -0.1083 & -0.0001 & 0.0000 & 1.6441 \\
\hline \multirow[t]{2}{*}{${ }^{2} \mathbf{C}_{\mathrm{Ib}}$} & 1.3992 & 0.6504 & -0.8469 & 0.0068 & -0.2079 & 0.0003 & -0.0001 & 1.7611 \\
\hline & $\mathrm{Fe}$ & OH proximal & $\mathrm{OH}$ distal & $\operatorname{ImH}(\mathrm{His} 20)$ & Porp & Asp136 & $\operatorname{Arg} 132$ & \\
\hline${ }^{2} \mathbf{C}_{\mathrm{R}} \mathbf{H}$ & 1.0451 & -0.0039 & 0.0005 & -0.0106 & -0.0308 & 0.0000 & 0.0000 & 0.8254 \\
\hline${ }^{4} \mathbf{C}_{\mathrm{R}} \mathbf{H}$ & 2.8312 & 0.0128 & 0.0041 & 0.1272 & 0.0245 & 0.0000 & -0.0001 & 3.7965 \\
\hline${ }^{6} \mathbf{C}_{\mathrm{R}} \mathbf{H}$ & 4.1748 & 0.0187 & 0.0013 & 0.1316 & 0.6730 & 0.0000 & 0.0000 & 8.7587 \\
\hline${ }^{2} \mathrm{TS}_{\mathrm{OO}} \mathrm{H}$ & 0.8065 & -0.1372 & -0.4538 & 0.0236 & 0.7675 & -0.0004 & -0.0001 & 1.3667 \\
\hline${ }^{2} \mathbf{C}_{\mathrm{Ia}} \mathbf{H}$ & 0.9121 & -0.1587 & -0.7324 & 0.0204 & 0.9653 & -0.0005 & 0.0000 & 1.6998 \\
\hline${ }^{2} \mathbf{C}_{\mathrm{lb}} \mathbf{H}$ & 0.9366 & 0.0609 & -0.9844 & 0.0186 & 0.9700 & 0.0001 & -0.0001 & 1.7698 \\
\hline
\end{tabular}

Table S20. The Calculated Mulliken Charge Distribution from the $287 \mathrm{ps}$ Snapshot at the QM/MM B2 Level.

\begin{tabular}{|l|c|c|c|c|c|c|c|}
\hline & Fe & O proximal & OH distal & ImH(His20) & Porp & Asp136 & Arg132 \\
\hline${ }^{2} \mathbf{C}_{\mathrm{R}}$ & 0.8292 & 0.1355 & -0.0694 & 0.4223 & -1.2339 & -0.8114 & 0.8333 \\
\hline${ }^{4} \mathbf{C}_{\mathrm{R}}$ & 1.1580 & -0.1269 & -0.1049 & 0.1379 & -0.9673 & -0.8087 & 0.8313 \\
\hline${ }^{6} \mathbf{C}_{\mathrm{R}}$ & 1.1734 & -0.0404 & -0.0863 & 0.1916 & -1.1447 & -0.8093 & 0.8338 \\
\hline${ }^{2} \mathbf{C}_{\mathrm{Ia}}$ & 1.0086 & 0.1134 & -0.0751 & 0.1462 & -1.0937 & -0.8049 & 0.8350 \\
\hline${ }^{2} \mathbf{C}_{\mathrm{Ib}}$ & 1.0340 & -0.0318 & -0.1347 & 0.1060 & -0.8707 & -0.7991 & 0.8425 \\
\hline & $\mathrm{Fe}$ & OH proximal & OH distal & ImH(His20) & Porp & Asp136 & Arg132 \\
\hline & 0.8515 & 0.3175 & -0.0825 & 0.9260 & -0.9644 & -0.8038 & 0.8642 \\
\hline${ }^{2} \mathbf{C}_{\mathrm{R}} \mathbf{H}$ & 1.1404 & 0.1408 & -0.0355 & 0.4054 & -0.5953 & -0.8054 & 0.8655 \\
\hline${ }^{4} \mathbf{C}_{\mathrm{R}} \mathbf{H}$ & 1.2348 & 0.1393 & -0.0555 & 0.5694 & -0.8324 & -0.8052 & 0.8652 \\
\hline${ }^{6} \mathbf{C}_{\mathrm{R}} \mathbf{H}$ & 0.5375 & 0.3080 & -0.1674 & 0.6842 & -0.2981 & -0.8106 & 0.8537 \\
\hline${ }^{2} \mathbf{T S} \mathbf{S O}_{\mathbf{O O}} \mathbf{H}$ & 0.7137 & 0.1063 & -0.1646 & 0.5642 & -0.1321 & -0.8061 & 0.8552 \\
\hline${ }^{2} \mathbf{C}_{\mathrm{Ia}} \mathbf{H}$ & 0.6292 & -0.1023 & 0.0130 & 0.5098 & 0.0420 & -0.8061 & 0.8597 \\
\hline${ }^{2} \mathbf{C}_{\mathrm{Ib}} \mathbf{H}$ & 0.023 \\
\hline
\end{tabular}




\section{Coordinates}

QM region coordinates are supplied here in xyz-format.

\section{Snapshot 0 ps}

$$
\begin{aligned}
& { }^{2} \mathbf{C}_{\mathrm{R}} \\
& \text { N } \quad-4.3954306 .102990-9.513772 \\
& \text { H } \quad-4.6820206 .834958-10.171786 \\
& \text { C } \quad-5.0936075 .604696-8.408975 \\
& \text { C } \quad-3.1851545 .474846-9.577275 \\
& \text { H } \quad-2.4559655 .658766-10.348288 \\
& \text { N } \quad-3.0750114 .604986-8.565186 \\
& \text { C } \quad-4.2574014 .678971-7.834776 \\
& \text { H } \quad-4.4211454 .051270-6.980336 \\
& \text { O } \quad-0.1261442 .289784-7.585141 \\
& \text { O } \quad 0.9869562 .914312-6.874888 \\
& \text { H } \quad 1.0140503 .788907-7.314803 \\
& \text { FE }-1.4829643 .434249-8.061245 \\
& \text { N } \quad-2.7292771 .867788-7.759246 \\
& \text { N } \quad-1.7082244 .029833-6.132475 \\
& \text { N } \quad-0.1949815 .005831-8.392364 \\
& \mathrm{~N} \quad-1.3021872 .889110-10.003342 \\
& \text { C } \quad-3.1232190 .907546-8.675561 \\
& \text { C } \quad-3.996680-0.073423-8.027497 \\
& \text { C } \quad-4.1281190 .310649-6.722536 \\
& \text { C } \quad-3.3180641 .519889-6.558277 \\
& \text { C } \quad-2.4070723 .356091-5.145012 \\
& \text { C } \quad-2.2495114 .037659-3.857863 \\
& \text { C } \quad-1.4282355 .111067-4.065784 \\
& \text { C } \quad-1.1187715 .113899-5.507451 \\
& \text { C } \quad 0.1261286 .003221-7.482843 \\
& \text { C } \quad 1.0617066 .940828-8.084138 \\
& \text { C } \quad 1.3160466 .514998-9.362206 \\
& \text { C } \quad 0.4756605 .329874-9.574102 \\
& \text { C } \quad-0.5670853 .547088-10.974661 \\
& \text { C } \quad-0.7300052 .882115-12.264811 \\
& \text { C } \quad-1.5500481 .810945-12.055741 \\
& \text { C } \quad-1.9125551 .818478-10.641327 \\
& \text { C } \quad-2.7420700 .896072-10.012723 \\
& \text { H } \quad-3.1370020 .091809-10.624224 \\
& \text { C } \quad-3.1521712 .200856-5.356071 \\
& \text { H } \quad-3.6402361 .759995-4.496080
\end{aligned}
$$

$\begin{array}{ll}\mathrm{H} & 2.0377176 .978902-10.034553 \\ \mathrm{H} & -0.2753363 .242690-13.187485 \\ \mathrm{H} & -1.9480121 .125750-12.804206 \\ \mathrm{H} & -0.5644241 .3793111 .136460 \\ \mathrm{H} & 3.004998-2.004870-3.194440\end{array}$

${ }^{4} \mathbf{C}_{\mathrm{R}}$

N $\quad-4.5421896 .144468-9.579007$

H $\quad-4.8365836 .879731-10.229105$

C $\quad-5.2109585 .639045-8.459629$

C $\quad-3.3216855 .534413-9.656978$

H $\quad-2.6052705 .726105-10.438855$

$\mathrm{N} \quad-3.1751654 .673715-8.644791$

C $\quad-4.3455864 .734029-7.895906$

$\mathrm{H} \quad-4.4803224 .106853-7.034766$

O $\quad 0.0820182 .197005-7.452753$

O $\quad 1.1877392 .893096-6.800486$

H $\quad 1.1561593 .760684-7.250024$

FE $\quad-1.4332853 .381001-8.074692$

N $\quad-2.6884931 .816487-7.757803$

N $\quad-1.7023974 .000136-6.143990$

N $\quad-0.1466174 .964489-8.410222$

$\mathrm{N} \quad-1.2503842 .843167-10.010901$

C $\quad-3.0790980 .856871-8.677003$

C $\quad-3.963880-0.112340-8.033403$

C $\quad-4.1127310 .281425-6.731702$

C $\quad-3.3017211 .485463-6.561597$

C $\quad-2.4134783 .338171-5.157692$

C $\quad-2.2571154 .026246-3.874651$

C $\quad-1.4194365 .087224-4.082428$

C $\quad-1.0982715 .078434-5.522598$

C $\quad 0.1639435 .959793-7.496910$

C $\quad 1.0860706 .908466-8.099534$

C $\quad 1.3376816 .491780-9.382007$

C $\quad 0.5108665 .299739-9.594504$

C $\quad-0.5319613 .514291-10.989467$

C $\quad-0.7115072 .854989-12.277413$

C $\quad-1.5231541 .777593-12.062171$

C $\quad-1.8685651 .773237-10.644689$

C $\quad-2.6946030 .847913-10.014077$ 


\begin{tabular}{|c|c|}
\hline $\mathrm{H}$ & $-3.0912290 .045793-10.626786$ \\
\hline $\mathrm{C}$ & $-3.1575172 .181568-5.365639$ \\
\hline $\mathrm{H}$ & $-3.6605281 .752274-4.508701$ \\
\hline $\mathrm{C}$ & $-0.2824355 .994901-6.180216$ \\
\hline $\mathrm{H}$ & $0.0995966 .815176-5.584069$ \\
\hline $\mathrm{C}$ & $0.2680244 .636991-10.793153$ \\
\hline $\mathrm{H}$ & $0.6848405 .065972-11.693592$ \\
\hline $\mathrm{N}$ & 0.3575651 .3979970 .750954 \\
\hline $\mathrm{H}$ & 0.8460340 .4964720 .736366 \\
\hline $\mathrm{C}$ & 1.0474872 .4810160 .245962 \\
\hline $\mathrm{N}$ & 0.7134903 .7420200 .488207 \\
\hline $\mathrm{H}$ & -0.0588503 .9854401 .114877 \\
\hline $\mathrm{H}$ & 1.2685614 .5778820 .086239 \\
\hline $\mathrm{N}$ & $2.1333202 .176746-0.484693$ \\
\hline $\mathrm{H}$ & $2.2660961 .239129-0.884636$ \\
\hline $\mathrm{H}$ & $2.7228982 .915910-0.842947$ \\
\hline $\mathrm{C}$ & $2.676497-1.527630-2.273571$ \\
\hline $\mathrm{O}$ & $2.508393-2.272415-1.261730$ \\
\hline $\mathrm{O}$ & $2.474485-0.217482-2.274886$ \\
\hline $\mathrm{O}$ & $0.2683361 .241950-2.797538$ \\
\hline $\mathrm{H}$ & $-0.5691490 .767355-2.951155$ \\
\hline $\mathrm{H}$ & $1.0111350 .586607-2.634985$ \\
\hline $\mathrm{O}$ & $3.6728141 .859478-3.391229$ \\
\hline $\mathrm{H}$ & $2.8960042 .350703-3.786071$ \\
\hline $\mathrm{H}$ & $3.3629590 .971870-3.046348$ \\
\hline $\mathrm{O}$ & $4.9263243 .751694-2.205167$ \\
\hline $\mathrm{H}$ & $4.5264092 .907488-2.632987$ \\
\hline $\mathrm{H}$ & $5.1590103 .603412-1.251154$ \\
\hline $\mathrm{O}$ & $1.9806665 .799462-0.394959$ \\
\hline $\mathrm{H}$ & $1.5527116 .630230-0.091519$ \\
\hline $\mathrm{H}$ & $2.2330575 .728842-1.385301$ \\
\hline $\mathrm{O}$ & $1.4568773 .168927-4.080872$ \\
\hline $\mathrm{H}$ & $0.8433682 .549611-3.568373$ \\
\hline $\mathrm{H}$ & $1.2513853 .126507-5.050323$ \\
\hline $\mathrm{O}$ & $2.7266765 .206019-2.746688$ \\
\hline $\mathrm{H}$ & $2.1660804 .565774-3.268239$ \\
\hline $\mathrm{H}$ & $3.6324994 .805738-2.583965$ \\
\hline $\mathrm{H}$ & $-6.1792445 .963110-8.078223$ \\
\hline $\mathrm{H}$ & $-4.385811-0.986018-8.530121$ \\
\hline $\mathrm{H}$ & $-4.680053-0.177479-5.921999$ \\
\hline $\mathrm{H}$ & $-2.7537403 .690772-2.964219$ \\
\hline
\end{tabular}

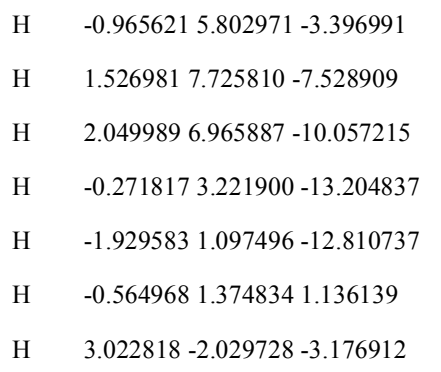

\section{${ }^{6} \mathbf{C}_{\mathrm{R}}$}

N $\quad-4.5012006 .128707-9.579207$

H $\quad-4.7925276 .866887-10.228231$

C $\quad-5.1754035 .628031-8.459960$

C $\quad-3.2885855 .506522-9.661310$

H $\quad-2.5703595 .694440-10.442403$

$\mathrm{N} \quad-3.1512674 .641578-8.649846$

C $\quad-4.3210864 .711736-7.899036$

H $\quad-4.4632444 .085149-7.038933$

O $\quad 0.0526252 .229384-7.422715$

O $\quad 1.1704312 .929560-6.826413$

H $\quad 1.1210663 .794487-7.285936$

FE $\quad-1.4355473 .363189-8.093520$

$\mathrm{N} \quad-2.7125031 .770535-7.778213$

$\mathrm{N} \quad-1.7358534 .028173-6.118111$

N $\quad-0.1156464 .979791-8.439447$

$\mathrm{N} \quad-1.2240302 .802678-10.086576$

C $\quad-3.0944830 .821170-8.706711$

C $\quad-3.979792-0.147382-8.053559$

C $\quad-4.1260530 .245565-6.749155$

C $\quad-3.3161291 .454660-6.574070$

C $\quad-2.4372883 .347035-5.145502$

C $\quad-2.2705064 .035495-3.857031$

C $\quad-1.4363095 .100686-4.066850$

C $\quad-1.1194375 .099279-5.512762$

C $\quad 0.1774495 .971690-7.516978$

C $\quad 1.0932926 .925215-8.128698$

C $\quad 1.3468026 .509208-9.413180$

C $\quad 0.5305415 .305439-9.630229$

C $\quad-0.5055153 .483813-11.051126$

C $\quad-0.6942662 .824280-12.344147$

C $\quad-1.5128171 .749855-12.129916$

C $\quad-1.8576541 .739031-10.706022$
C $\quad-2.6950540 .824659-10.051494$

H $\quad-3.0998470 .025134-10.664630$

C $\quad-3.1691682 .174432-5.379228$

H $\quad-3.6741101 .742068-4.523140$

C $\quad-0.2855375 .998489-6.194364$

H $\quad 0.0994616 .820802-5.600467$

C $\quad 0.2884904 .618235-10.828566$

H $\quad 0.7086115 .048586-11.728001$

$\mathrm{N} \quad 0.3540061 .3939950 .743329$

H $\quad 0.8462780 .4945400 .730483$

C $\quad 1.0403292 .4791820 .237449$

N $\quad 0.7049223 .7392030 .481237$

H $\quad-0.0660403 .9816461 .110094$

$\mathrm{H} \quad 1.2604364 .5766770 .081024$

N $\quad 2.1240852 .177391-0.497549$

H $\quad 2.2567181 .240824-0.900588$

H $\quad 2.7098792 .918388-0.857986$

C $\quad 2.678223-1.519859-2.286915$

O $\quad 2.518691-2.261547-1.271560$

O $\quad 2.473145-0.210019-2.291840$

O $\quad 0.2731061 .245271-2.834975$

H $\quad-0.5743870 .783846-2.969895$

H $\quad 1.0099820 .584530-2.666606$

O $\quad 3.6792591 .874185-3.396168$

H $\quad 2.9025882 .365462-3.790148$

H $\quad 3.3704150 .985761-3.053464$

O $\quad 4.9440423 .774879-2.226099$

H $\quad 4.5421352 .928880-2.646255$

H $\quad 5.1773493 .630676-1.271379$

O $\quad 1.9757495 .796308-0.391846$

H $\quad 1.5495576 .627472-0.086943$

H $\quad 2.2351385 .730489-1.380919$

O $\quad 1.4618223 .183429-4.083205$

H $\quad 0.8469732 .554544-3.580827$

H $\quad 1.2523583 .161453-5.049206$

O $\quad 2.7317325 .216230-2.741750$

H $\quad 2.1703624 .578190-3.264027$

H $\quad 3.6414394 .820570-2.588687$

H $\quad-6.1462245 .952373-8.085292$

H $\quad-4.405769-1.018035-8.552130$

H $\quad-4.692394-0.213544-5.938881$ 


\begin{tabular}{|c|c|}
\hline $\mathrm{H}$ & $-2.7596823 .693204-2.945105$ \\
\hline $\mathrm{H}$ & $-0.9793115 .819654-3.386921$ \\
\hline $\mathrm{H}$ & $1.5283307 .746476-7.559192$ \\
\hline $\mathrm{H}$ & $2.0542856 .989564-10.089043$ \\
\hline $\mathrm{H}$ & $-0.2592033 .197753-13.271134$ \\
\hline $\mathrm{H}$ & $-1.9280881 .073843-12.877328$ \\
\hline $\mathrm{H}$ & -0.5666311 .3690501 .132913 \\
\hline $\mathrm{H}$ & $3.018932-2.024840-3.190783$ \\
\hline \multicolumn{2}{|c|}{${ }^{2} \mathbf{C}_{\text {Ia }}$} \\
\hline $\mathrm{N}$ & $-4.4248846 .110013-9.537808$ \\
\hline $\mathrm{H}$ & $-4.7132656 .844529-10.192639$ \\
\hline $\mathrm{C}$ & $-5.1136455 .613914-8.425480$ \\
\hline $\mathrm{C}$ & $-3.2163775 .480435-9.610473$ \\
\hline $\mathrm{H}$ & $-2.4925585 .664605-10.386643$ \\
\hline $\mathrm{N}$ & $-3.0951104 .612446-8.597674$ \\
\hline $\mathrm{C}$ & $-4.2717994 .690544-7.856897$ \\
\hline $\mathrm{H}$ & $-4.4287064 .064120-7.000217$ \\
\hline $\mathrm{O}$ & $-0.2373542 .361351-7.711304$ \\
\hline $\mathrm{O}$ & $1.7811223 .393388-6.543130$ \\
\hline $\mathrm{H}$ & $1.1448183 .857297-7.127640$ \\
\hline FE & $-1.4537473 .382606-8.092324$ \\
\hline $\mathrm{N}$ & $-2.7768501 .882886-7.783885$ \\
\hline $\mathrm{N}$ & $-1.7116944 .003642-6.157074$ \\
\hline $\mathrm{N}$ & $-0.2170414 .993241-8.440013$ \\
\hline $\mathrm{N}$ & $-1.3258342 .892837-10.046698$ \\
\hline $\mathrm{C}$ & $-3.1592900 .921162-8.702361$ \\
\hline $\mathrm{C}$ & $-4.020696-0.067650-8.051475$ \\
\hline $\mathrm{C}$ & $-4.1509720 .311183-6.744658$ \\
\hline $\mathrm{C}$ & $-3.3500261 .524490-6.578159$ \\
\hline $\mathrm{C}$ & $-2.4123093 .339958-5.164949$ \\
\hline $\mathrm{C}$ & $-2.2411464 .021486-3.880050$ \\
\hline $\mathrm{C}$ & $-1.4092705 .085431-4.091970$ \\
\hline $\mathrm{C}$ & $-1.1100525 .085931-5.537815$ \\
\hline $\mathrm{C}$ & $0.1155595 .976500-7.525552$ \\
\hline $\mathrm{C}$ & $1.0656976 .907594-8.120791$ \\
\hline $\mathrm{C}$ & $1.3241146 .479209-9.396499$ \\
\hline $\mathrm{C}$ & $0.4712485 .304750-9.614450$ \\
\hline $\mathrm{C}$ & $-0.5752283 .532937-11.014529$ \\
\hline $\mathrm{C}$ & $-0.7371442 .858312-12.298450$ \\
\hline $\mathrm{C}$ & $-1.5677161 .796549-12.084078$ \\
\hline
\end{tabular}

$$
\begin{aligned}
& \text { C } \quad-1.9392981 .818657-10.671851 \\
& \text { C } \quad-2.7749910 .906027-10.038249 \\
& \mathrm{H} \quad-3.1673490 .098198-10.646258 \\
& \text { C } \quad-3.1716562 .194429-5.373145 \\
& \mathrm{H} \quad-3.6558391 .753071-4.511472 \\
& \text { C } \quad-0.3157416 .011925-6.203487 \\
& \text { H } \quad 0.0680346 .835131-5.612681 \\
& \text { C } \quad 0.2402544 .642374-10.812593 \\
& \text { H } \quad 0.6832685 .055741-11.707489 \\
& \text { N } \quad 0.3604571 .4172920 .745497 \\
& \mathrm{H} \quad 0.8447980 .5137980 .723634 \\
& \text { C } \quad 1.0644012 .5047300 .268059 \\
& \text { N } \quad 0.7365873 .7635650 .526611 \\
& \text { H } \quad-0.0482524 .0004971 .140639 \\
& \mathrm{H} \quad 1.3102964 .6096770 .165217 \\
& \text { N } \quad 2.1561212 .202924-0.455001 \\
& \text { H } \quad 2.2871181 .269980-0.866648 \\
& \text { H } \quad 2.7524502 .942738-0.799725 \\
& \text { C } \quad 2.683186-1.477915-2.285292 \\
& \text { O } \quad 2.510105-2.232425-1.281341 \\
& \text { O } \quad 2.494143-0.166443-2.273416 \\
& \text { O } \quad 0.3028011 .300928-2.824375 \\
& \mathrm{H} \quad-0.5398400 .829925-2.954330 \\
& \mathrm{H} \quad 1.0464490 .646757-2.660922 \\
& \text { O } \quad 3.7305171 .947424-3.289819 \\
& \text { H } \quad 2.9462442 .474299-3.616808 \\
& \text { H } \quad 3.4214361 .044925-2.990444 \\
& \text { O } \quad 5.0536303 .808570-2.121692 \\
& \text { H } \quad 4.6340602 .977786-2.551940 \\
& \text { H } \quad 5.2764613 .640839-1.168145 \\
& \text { O } \quad 2.0317335 .843941-0.237778 \\
& \text { H } \quad 1.5743216 .6658340 .049651 \\
& \text { H } \quad 2.3561015 .798593-1.211472 \\
& \text { O } \quad 1.5374903 .352447-3.842076 \\
& \text { H } \quad 0.8862092 .689843-3.448056 \\
& \mathrm{H} \quad 1.4500453 .417037-4.826912 \\
& \text { O } \quad 2.8726835 .314229-2.560425 \\
& \text { H } \quad 2.2818794 .674547-3.053238 \\
& \text { H } \quad 3.7779404 .900683-2.431633 \\
& \text { H } \quad-6.0912305 .937516-8.068161 \\
& \text { H } \quad-4.431155-0.947677-8.546592
\end{aligned}
$$

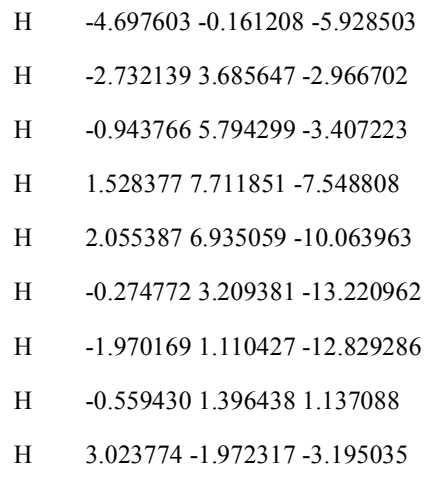




\begin{tabular}{|c|c|}
\hline $\mathrm{C}$ & $-1.6096961 .760286-12.108801$ \\
\hline $\mathrm{C}$ & $-1.9609081 .776623-10.692233$ \\
\hline $\mathrm{C}$ & $-2.7805320 .856946-10.050497$ \\
\hline $\mathrm{H}$ & $-3.1689630 .043675-10.653548$ \\
\hline $\mathrm{C}$ & $-3.2207872 .181890-5.396394$ \\
\hline $\mathrm{H}$ & $-3.7135851 .745861-4.536580$ \\
\hline $\mathrm{C}$ & $-0.2170545 .877949-6.205909$ \\
\hline $\mathrm{H}$ & $0.2550586 .642702-5.601098$ \\
\hline $\mathrm{C}$ & $0.1660684 .639086-10.869120$ \\
\hline $\mathrm{H}$ & $0.5941935 .055558-11.769330$ \\
\hline $\mathrm{N}$ & 0.3585051 .4192700 .741632 \\
\hline $\mathrm{H}$ & 0.8401770 .5143680 .714711 \\
\hline $\mathrm{C}$ & 1.0733682 .5099790 .287215 \\
\hline $\mathrm{N}$ & 0.7503233 .7667000 .559801 \\
\hline $\mathrm{H}$ & -0.0454224 .0013541 .160955 \\
\hline $\mathrm{H}$ & 1.3377474 .6155790 .225341 \\
\hline $\mathrm{N}$ & $2.1690932 .211605-0.431383$ \\
\hline $\mathrm{H}$ & $2.2996051 .282028-0.850574$ \\
\hline $\mathrm{H}$ & $2.7671332 .953540-0.768943$ \\
\hline $\mathrm{C}$ & $2.698948-1.464238-2.280650$ \\
\hline $\mathrm{O}$ & $2.523565-2.222115-1.279721$ \\
\hline $\mathrm{O}$ & $2.520335-0.151735-2.261726$ \\
\hline $\mathrm{O}$ & $0.3608931 .326965-2.888428$ \\
\hline $\mathrm{H}$ & $-0.4917930 .864680-2.974089$ \\
\hline $\mathrm{H}$ & $1.0976420 .671196-2.703969$ \\
\hline $\mathrm{O}$ & $3.7713731 .964339-3.251869$ \\
\hline $\mathrm{H}$ & $2.9995512 .507840-3.582821$ \\
\hline $\mathrm{H}$ & $3.4516731 .064313-2.957198$ \\
\hline $\mathrm{O}$ & $5.1124033 .785926-2.040616$ \\
\hline $\mathrm{H}$ & $4.6806912 .970124-2.487964$ \\
\hline $\mathrm{H}$ & $5.3307213 .598220-1.089453$ \\
\hline $\mathrm{O}$ & $2.0660425 .851472-0.144859$ \\
\hline $\mathrm{H}$ & 1.5900166 .6695180 .123775 \\
\hline $\mathrm{H}$ & $2.4221885 .805926-1.109127$ \\
\hline $\mathrm{O}$ & $1.6231323 .425829-3.789792$ \\
\hline $\mathrm{H}$ & $0.9549722 .756594-3.442219$ \\
\hline $\mathrm{H}$ & $1.5279573 .576025-4.770342$ \\
\hline $\mathrm{O}$ & $2.9593295 .324506-2.440257$ \\
\hline $\mathrm{H}$ & $2.3695944 .696838-2.950924$ \\
\hline $\mathrm{H}$ & $3.8623054 .903523-2.317009$ \\
\hline $\mathrm{H}$ & $-6.1060375 .932975-8.075119$ \\
\hline
\end{tabular}

\begin{tabular}{|c|c|c|c|}
\hline $\mathrm{H}$ & $-4.445645-0.982861-8.558905$ & $\mathrm{C}$ & $-0.8657502 .880421-12.352310$ \\
\hline $\mathrm{H}$ & $-4.723171-0.185325-5.946052$ & $\mathrm{C}$ & $-1.6012971 .753416-12.105302$ \\
\hline $\mathrm{H}$ & $-2.7606823 .667474-2.987151$ & $\mathrm{C}$ & $-1.9031551 .740849-10.681325$ \\
\hline $\mathrm{H}$ & $-0.8946995 .709064-3.413756$ & $\mathrm{C}$ & $-2.6887230 .796216-10.018122$ \\
\hline $\mathrm{H}$ & $1.5784527 .605759-7.557574$ & $\mathrm{H}$ & $-3.053750-0.035823-10.610830$ \\
\hline $\mathrm{H}$ & $2.0297156 .897825-10.110375$ & $\mathrm{C}$ & $-3.3207862 .275376-5.422417$ \\
\hline $\mathrm{H}$ & $-0.3498233 .187930-13.264318$ & $\mathrm{H}$ & $-3.8850491 .891308-4.579911$ \\
\hline $\mathrm{H}$ & $-2.0141521 .072525-12.851409$ & $\mathrm{C}$ & $0.4383015 .195984-6.125889$ \\
\hline $\mathrm{H}$ & -0.5568371 .4007541 .143849 & $\mathrm{H}$ & $0.9495325 .936603-5.510314$ \\
\hline $\mathrm{H}$ & $3.032655-1.956790-3.193940$ & $\mathrm{C}$ & $0.0479034 .731339-10.909769$ \\
\hline & & $\mathrm{H}$ & $0.3829385 .195347-11.826610$ \\
\hline${ }^{2} \mathbf{P}$ & & $\mathrm{N}$ & 0.3761741 .4238890 .773882 \\
\hline $\mathrm{N}$ & $-4.3696416 .109872-9.520296$ & $\mathrm{H}$ & 0.8478090 .5142750 .743026 \\
\hline $\mathrm{H}$ & $-4.6587196 .841696-10.177765$ & $\mathrm{C}$ & 1.1001132 .5084510 .323966 \\
\hline $\mathrm{C}$ & $-5.0670535 .611081-8.415907$ & $\mathrm{~N}$ & 0.7731673 .7681800 .578883 \\
\hline $\mathrm{C}$ & $-3.1639745 .475428-9.590270$ & $\mathrm{H}$ & -0.0316934 .0071271 .165743 \\
\hline $\mathrm{H}$ & $-2.4399425 .663458-10.364114$ & $\mathrm{H}$ & 1.3617514 .6123410 .243856 \\
\hline $\mathrm{N}$ & $-3.0486254 .598072-8.582960$ & $\mathrm{~N}$ & $2.2147862 .203389-0.363912$ \\
\hline $\mathrm{C}$ & $-4.2321304 .679710-7.849328$ & $\mathrm{H}$ & $2.3476241 .274307-0.778976$ \\
\hline $\mathrm{H}$ & $-4.4002644 .051045-6.997680$ & $\mathrm{H}$ & $2.8304522 .939504-0.680666$ \\
\hline $\mathrm{O}$ & $-0.1416132 .333401-7.710376$ & $\mathrm{C}$ & $2.699049-1.509395-2.265075$ \\
\hline $\mathrm{O}$ & $1.3694914 .038802-6.104626$ & $\mathrm{O}$ & $2.513925-2.267689-1.266167$ \\
\hline $\mathrm{H}$ & $0.9461293 .341968-6.672560$ & $\mathrm{O}$ & $2.535898-0.195399-2.242834$ \\
\hline FE & $-1.4086333 .296208-8.119723$ & $\mathrm{O}$ & $0.3569751 .275293-2.693777$ \\
\hline $\mathrm{N}$ & $-2.7583171 .832673-7.789095$ & $\mathrm{H}$ & $-0.4880650 .821688-2.861085$ \\
\hline $\mathrm{N}$ & $-1.6285163 .885703-6.159948$ & $\mathrm{H}$ & $1.0997560 .609301-2.564678$ \\
\hline $\mathrm{N}$ & $-0.1503584 .880425-8.489761$ & $\mathrm{O}$ & $3.8192941 .908714-3.230620$ \\
\hline $\mathrm{N}$ & $-1.3280592 .844757-10.073789$ & $\mathrm{H}$ & $3.0162532 .447858-3.492172$ \\
\hline $\mathrm{C}$ & $-3.1070220 .838775-8.686674$ & $\mathrm{H}$ & $3.5131141 .007048-2.929228$ \\
\hline $\mathrm{C}$ & $-4.004419-0.114536-8.044641$ & $\mathrm{O}$ & $5.1155993 .783080-2.038899$ \\
\hline $\mathrm{C}$ & $-4.2030640 .321070-6.760950$ & $\mathrm{H}$ & $4.7051042 .952064-2.479311$ \\
\hline $\mathrm{C}$ & $-3.4129661 .533942-6.602492$ & $\mathrm{H}$ & $5.3442223 .601196-1.088929$ \\
\hline $\mathrm{C}$ & $-2.5230683 .385016-5.198018$ & $\mathrm{O}$ & $2.0979565 .859878-0.118294$ \\
\hline $\mathrm{C}$ & $-2.3114824 .072725-3.938994$ & $\mathrm{H}$ & 1.6111216 .6726310 .149739 \\
\hline $\mathrm{C}$ & $-1.2509494 .934009-4.118156$ & $\mathrm{H}$ & $2.4520735 .826028-1.079812$ \\
\hline $\mathrm{C}$ & $-0.8448954 .770899-5.500934$ & $\mathrm{O}$ & $1.6395783 .322445-3.575616$ \\
\hline $\mathrm{C}$ & $0.3790295 .670351-7.531380$ & $\mathrm{H}$ & $0.9690612 .656968-3.213015$ \\
\hline $\mathrm{C}$ & $1.1691026 .723404-8.098053$ & $\mathrm{H}$ & $1.4840203 .533969-4.535850$ \\
\hline $\mathrm{C}$ & $1.2220086 .504488-9.458920$ & $\mathrm{O}$ & $2.9677055 .336143-2.439980$ \\
\hline $\mathrm{C}$ & $0.3656495 .353714-9.713471$ & $\mathrm{H}$ & $2.3660774 .642804-2.843778$ \\
\hline $\mathrm{C}$ & $-0.6863913 .555569-11.078985$ & $\mathrm{H}$ & $3.8757974 .923217-2.320913$ \\
\hline
\end{tabular}




$$
\begin{array}{ll}
\mathrm{H} & -6.0492745 .933381-8.070319 \\
\mathrm{H} & -4.418844-0.996122-8.533644 \\
\mathrm{H} & -4.783838-0.125500-5.953891 \\
\mathrm{H} & -2.8961393 .851048-3.046188 \\
\mathrm{H} & -0.7206935 .598976-3.436458 \\
\mathrm{H} & 1.6347417 .483438-7.470682 \\
\mathrm{H} & 1.8728817 .057830-10.135856 \\
\mathrm{H} & -0.4717853 .264026-13.293428 \\
\mathrm{H} & -2.0027061 .060583-12.844843 \\
\mathrm{H} & -0.5541421 .4083791 .140275 \\
\mathrm{H} & 3.028010-1.999515-3.181390
\end{array}
$$

\section{${ }^{2} \mathbf{C}_{\mathrm{R}} \mathbf{H}$}

$$
\begin{aligned}
& \text { N } \quad-4.4357736 .098489-9.537633 \\
& \text { H } \quad-4.7168726 .836319-10.200167 \\
& \text { C } \quad-5.1467665 .603368-8.436690 \\
& \text { C } \quad-3.2285585 .487331-9.595034 \\
& \text { H } \quad-2.4931155 .671325-10.360499 \\
& \mathrm{~N} \quad-3.1294644 .623544-8.566321 \\
& \text { C } \quad-4.3190314 .688163-7.843568 \\
& \text { H } \quad-4.4903994 .062647-6.989903 \\
& \text { O } \quad-0.0136342 .292962-7.290581 \\
& \text { O } \quad 1.1849333 .059773-6.975240 \\
& \text { H } \quad 0.9426803 .892828-7.461530 \\
& \text { FE } \quad-1.6391253 .541155-8.052805 \\
& \mathrm{~N} \quad-2.7531091 .910228-7.665317 \\
& \mathrm{~N} \quad-1.7767774 .055938-6.086773 \\
& \mathrm{~N} \quad-0.2415525 .005606-8.359908 \\
& \mathrm{~N} \quad-1.3239812 .911586-9.929047 \\
& \text { C } \quad-3.1486360 .939021-8.584180 \\
& \text { C } \quad-4.024666-0.034040-7.940713 \\
& \text { C } \quad-4.1824140 .360820-6.640943 \\
& \text { C } \quad-3.3880931 .569678-6.469374 \\
& \text { C } \quad-2.5062953 .421325-5.080366 \\
& \text { C } \quad-2.3240784 .107854-3.808814 \\
& \text { C } \quad-1.4575525 .145184-4.025907 \\
& \text { C } \quad-1.1594665 .138263-5.465814 \\
& \text { C } \quad 0.0709876 .025081-7.452733 \\
& \text { C } \quad 1.0019826 .956551-8.058753 \\
& \text { C } \quad 1.2539946 .532569-9.339512 \\
& \text { C } \quad 0.4236885 .352118-9.554183
\end{aligned}
$$

$$
\begin{array}{lllll}
\text { C } & -0.6096953 .565072-10.930275 & \text { H } & 2.3583614 .715224-3.128264 \\
\text { C } & -0.7893142 .887672-12.205101 & \text { H } & 3.8136634 .872492-2.401240 \\
\text { C } & -1.6014611 .814419-11.973485 & \text { H } & -6.1258685 .932980-8.089156 \\
\text { C } & -1.9443901 .832754-10.557412 & \text { H } & -4.443050-0.908747-8.438617 \\
\text { C } & -2.7657000 .914677-9.918127 & \text { H } & -4.750491-0.101830-5.833906 \\
\text { H } & -3.1601880 .106188-10.522228 & \text { H } & -2.8401873 .801620-2.898914 \\
\text { C } & -3.2569112 .268459-5.276725 & \text { H } & -0.9686415 .836620-3.339650 \\
\text { H } & -3.7650381 .847491-4.419202 & \text { H } & 1.4677107 .764031-7.493828 \\
\text { C } & -0.3552856 .056776-6.132790 & \text { H } & 1.9722956 .997195-10.014963 \\
\text { H } & 0.0249056 .881251-5.543242 & \text { H } & -0.3465563 .235576-13.138372 \\
\text { C } & 0.1859744 .689705-10.749533 & \text { H } & -1.9972811 .116130-12.710902 \\
\text { H } & 0.6015495 .114990-11.651607 & \text { H } & -0.5963691 .3344691 .137996 \\
\text { N } & 0.2890331 .3674530 .674371 & \text { H } & 2.963309-1.936588-3.270257
\end{array}
$$

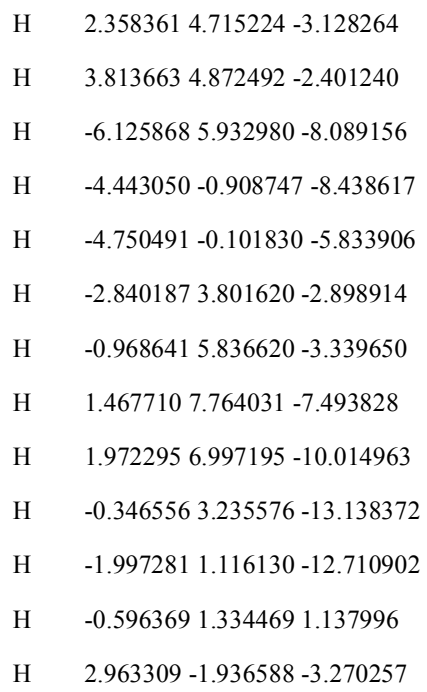$$
\mathrm{H} \quad 0.8052210 .4804700 .677140
$$$$
\text { C } \quad 0.9789882 .4710430 .208614
$$$$
\mathrm{N} \quad 0.6718243 .7230440 .508913
$$$$
\text { H } \quad-0.0809883 .9592451 .164329
$$$$
\text { H } \quad 1.2414444 .5733530 .143346
$$$$
\text { N } \quad 2.0243052 .186483-0.590447
$$$$
\text { H } \quad 2.1582781 .254899-1.006185
$$$$
\mathrm{H} \quad 2.6460452 .924710-0.891830
$$$$
\text { C } \quad 2.586328-1.427189-2.383429
$$$$
\text { O } \quad 2.384543-2.169917-1.385726
$$$$
\text { O } \quad 2.389238-0.114839-2.411054
$$$$
\text { H } \quad-0.3611962 .118039-6.391577
$$$$
\text { O } \quad 0.3405751 .267310-3.402499
$$$$
\text { H } \quad-0.5218681 .004868-3.035024
$$$$
\text { H } \quad 1.0612030 .628644-3.091138
$$$$
\text { O } \quad 3.7573001 .938325-3.395052
$$$$
\mathrm{H} \quad 3.0245242 .487722-3.776051
$$$$
\text { H } \quad 3.3968411 .061729-3.078606
$$$$
\text { O } \quad 5.0420293 .774853-2.101530
$$$$
\text { H } \quad 4.6549432 .950751-2.554044
$$$$
\text { H } \quad 5.3001243 .587784-1.158326
$$$$
\text { O } \quad 1.9637525 .791816-0.258909
$$$$
\text { H } \quad 1.5799996 .6307730 .082807
$$$$
\text { H } \quad 2.3360695 .785260-1.206124
$$$$
\text { O } \quad 1.5887433 .402222-4.099629
$$$$
\mathrm{H} \quad 0.9582512 .691177-3.737803
$$$$
\mathrm{H} \quad 1.4726613 .485857-5.066683
$$$$
\text { O } \quad 2.9204755 .307933-2.568126
$$

\section{${ }^{4} \mathbf{C}_{\mathrm{R}} \mathbf{H}$}

$$
\begin{aligned}
& \text { N } \quad-4.5892766 .143195-9.597793 \\
& \mathrm{H} \quad-4.8699506 .890795-10.247017 \\
& \text { C } \quad-5.2745185 .634968-8.489935 \\
& \text { C } \quad-3.3757335 .537801-9.674670 \\
& \text { H } \quad-2.6518075 .735455-10.448392 \\
& \mathrm{~N} \quad-3.2499284 .669687-8.658551 \\
& \text { C } \quad-4.4263594 .723744-7.917467 \\
& \text { H } \quad-4.5813364 .089119-7.065688 \\
& \text { O } \quad 1.0024262 .081221-6.382121 \\
& \text { O } \quad 1.6907253 .359004-6.446812 \\
& \text { H } \quad 1.0950193 .852244-7.049954 \\
& \text { FE } \quad-1.6369153 .495697-8.080280 \\
& \text { N } \quad-2.7029911 .834541-7.693666 \\
& \mathrm{~N} \quad-1.7211913 .982632-6.109397 \\
& \mathrm{~N} \quad-0.1799744 .904199-8.338511 \\
& \mathrm{~N} \quad-1.2442072 .798939-9.914971 \\
& \text { C } \quad-3.1012320 .854743-8.607220 \\
& \text { C } \quad-3.989958-0.101619-7.963968 \\
& \text { C } \quad-4.1513700 .304747-6.667978 \\
& \text { C } \quad-3.3431401 .503795-6.499250 \\
& \text { C } \quad-2.4409333 .334259-5.105920 \\
& \text { C } \quad-2.2729994 .020048-3.831344 \\
& \text { C } \quad-1.4278505 .076938-4.042997 \\
& \text { C } \quad-1.1252485 .074958-5.478576 \\
& \text { C } \quad 0.1149275 .942343-7.447607 \\
& \text { C } \quad 1.0447336 .871918-8.053044
\end{aligned}
$$




\begin{tabular}{|c|c|}
\hline $\mathrm{C}$ & $1.3256466 .423254-9.318941$ \\
\hline $\mathrm{C}$ & $0.5108075 .230523-9.523139$ \\
\hline $\mathrm{C}$ & $-0.5181113 .455036-10.910409$ \\
\hline $\mathrm{C}$ & $-0.7028852 .794310-12.188805$ \\
\hline $\mathrm{C}$ & $-1.5298511 .727386-11.971937$ \\
\hline $\mathrm{C}$ & $-1.8738331 .729409-10.561909$ \\
\hline $\mathrm{C}$ & $-2.7120710 .819144-9.936879$ \\
\hline $\mathrm{H}$ & $-3.1111460 .015433-10.544074$ \\
\hline $\mathrm{C}$ & $-3.2049012 .194082-5.304920$ \\
\hline $\mathrm{H}$ & $-3.7218621 .779110-4.450182$ \\
\hline $\mathrm{C}$ & $-0.3372616 .005837-6.139960$ \\
\hline $\mathrm{H}$ & $0.0115796 .850404-5.560237$ \\
\hline $\mathrm{C}$ & $0.2865864 .569057-10.719955$ \\
\hline $\mathrm{H}$ & $0.7173424 .991174-11.616076$ \\
\hline $\mathrm{N}$ & 0.2690121 .4011970 .629099 \\
\hline $\mathrm{H}$ & 0.7911260 .5179610 .632195 \\
\hline $\mathrm{C}$ & 0.9692832 .5144440 .202999 \\
\hline $\mathrm{N}$ & 0.6735943 .7588180 .533742 \\
\hline $\mathrm{H}$ & -0.0858553 .9830271 .187456 \\
\hline $\mathrm{H}$ & 1.2522104 .6263080 .202157 \\
\hline $\mathrm{N}$ & $2.0183772 .244163-0.599699$ \\
\hline $\mathrm{H}$ & $2.1495791 .324464-1.039784$ \\
\hline $\mathrm{H}$ & $2.6745322 .974789-0.838835$ \\
\hline $\mathrm{C}$ & $2.565893-1.302297-2.457236$ \\
\hline $\mathrm{O}$ & $2.339964-2.019484-1.448977$ \\
\hline $\mathrm{O}$ & $2.3847650 .015516-2.516413$ \\
\hline $\mathrm{H}$ & $0.8530491 .940234-5.402316$ \\
\hline $\mathrm{O}$ & $0.5942351 .487771-3.734978$ \\
\hline $\mathrm{H}$ & $-0.3180971 .265613-3.471325$ \\
\hline $\mathrm{H}$ & $1.2555890 .822292-3.316992$ \\
\hline $\mathrm{O}$ & $3.8659952 .119165-3.245711$ \\
\hline $\mathrm{H}$ & $3.1717862 .751927-3.552719$ \\
\hline $\mathrm{H}$ & $3.4756251 .228182-3.031792$ \\
\hline $\mathrm{O}$ & $5.2785233 .826773-1.891794$ \\
\hline $\mathrm{H}$ & $4.8452703 .049329-2.372924$ \\
\hline $\mathrm{H}$ & $5.5047053 .580084-0.953644$ \\
\hline $\mathrm{O}$ & $1.9634805 .851458-0.098587$ \\
\hline $\mathrm{H}$ & 1.5652016 .6815410 .250791 \\
\hline $\mathrm{H}$ & $2.5476515 .909759-0.933762$ \\
\hline $\mathrm{O}$ & $1.8349383 .865442-3.775531$ \\
\hline $\mathrm{H}$ & $1.1788723 .164701-3.513634$ \\
\hline
\end{tabular}

$$
\begin{array}{ll}
\mathrm{H} & 1.8019853 .963372-4.755916 \\
\mathrm{O} & 3.2999995 .556070-2.221608 \\
\mathrm{H} & 2.7100405 .035211-2.821983 \\
\mathrm{H} & 4.1574365 .048955-2.076803 \\
\mathrm{H} & -6.2392855 .966357-8.105914 \\
\mathrm{H} & -4.420694-0.971428-8.459918 \\
\mathrm{H} & -4.733850-0.144575-5.863681 \\
\mathrm{H} & -2.8020113 .721322-2.926371 \\
\mathrm{H} & -0.9614885 .781976-3.354888 \\
\mathrm{H} & 1.4848407 .701605-7.499879 \\
\mathrm{H} & 2.0423616 .891497-9.993577 \\
\mathrm{H} & -0.2597313 .151941-13.118204 \\
\mathrm{H} & -1.9407571 .049836-12.720367 \\
\mathrm{H} & -0.6202891 .3570121 .084252 \\
\mathrm{H} & 2.956553-1.837709-3.322560 \\
\hline &
\end{array}
$$

\section{${ }^{6} \mathrm{C}_{\mathrm{R}} \mathrm{H}$}

$$
\begin{aligned}
& \text { N } \quad-4.6105156 .148445-9.609641 \\
& \text { H } \quad-4.8933126 .899981-10.254868 \\
& \text { C } \quad-5.2901615 .634341-8.500013 \\
& \text { C } \quad-3.3991595 .548189-9.698879 \\
& \text { H } \quad-2.6764285 .750559-10.472604 \\
& \text { N } \quad-3.2686474 .674952-8.686019 \\
& \text { C } \quad-4.4417274 .720032-7.936784 \\
& \text { H } \quad-4.5845864 .083992-7.083792 \\
& \text { O } \quad 0.2892482 .208761-7.078628 \\
& \text { O } \quad 1.3949853 .123090-6.851951 \\
& \text { H } \quad 1.0621503 .886898-7.387054 \\
& \text { FE } \quad-1.6699513 .537165-8.124963 \\
& \text { N } \quad-2.6924161 .798955-7.696462 \\
& \mathrm{~N} \quad-1.7328424 .021887-6.085075 \\
& \mathrm{~N} \quad-0.1379964 .967245-8.375458 \\
& \text { N } \quad-1.2048992 .800110-9.984221 \\
& \text { C } \quad-3.0716500 .825631-8.621144 \\
& \text { C } \quad-3.960756-0.134268-7.974470 \\
& \text { C } \quad-4.1218650 .265525-6.674270 \\
& \text { C } \quad-3.3248181 .475233-6.497542 \\
& \text { C } \quad-2.4531103 .362534-5.092524 \\
& \text { C } \quad-2.2889314 .060673-3.820092 \\
& \text { C } \quad-1.4415225 .117290-4.033957 \\
& \text { C } \quad-1.1241135 .110674-5.470240
\end{aligned}
$$

C $\quad 0.1440275 .993004-7.467736$

C $\quad 1.0525996 .940000-8.084778$

C $\quad 1.3173366 .509841-9.362869$

C $\quad 0.5189985 .301730-9.572398$

C $\quad-0.4919103 .474405-10.972326$

C $\quad-0.6860592 .808395-12.251972$

C $\quad-1.5008321 .730911-12.028609$

C $\quad-1.8340631 .722986-10.610390$

C $\quad-2.6667370 .810825-9.957671$

H $\quad-3.0633060 .003967-10.564690$

C $\quad-3.1938492 .196763-5.308733$

Н $\quad-3.7033781 .774921-4.451109$

C $\quad-0.3118796 .024326-6.149177$

H $\quad 0.0463886 .863396-5.565085$

C $\quad 0.2907434 .613626-10.766003$

H $\quad 0.7081995 .043162-11.666254$

$\mathrm{N} \quad 0.2893351 .3569790 .677508$

H $\quad 0.8105320 .4728950 .683426$

C $\quad 0.9725762 .4626830 .206853$

$\mathrm{N} \quad 0.6590153 .7139410 .501550$

H $\quad-0.0922603 .9491981 .159273$

$\mathrm{H} \quad 1.2238354 .5695340 .134526$

N $\quad 2.0192052 .180243-0.591787$

H $\quad 2.1586131 .248639-1.004743$

H $2.6389562 .919704-0.893584$

C $\quad 2.617324-1.435567-2.381345$

O $\quad 2.410163-2.171641-1.379485$

O $\quad 2.413930-0.124693-2.420996$

$\mathrm{H} \quad-0.0579752 .104658-6.170014$

O $\quad 0.3753201 .255772-3.449502$

H $\quad-0.4853890 .975975-3.089072$

H $\quad 1.1012800 .624633-3.132372$

O $\quad 3.7800011 .957015-3.340055$

H $3.0419412 .509270-3.705562$

$\mathrm{H} \quad 3.4313531 .068230-3.047267$

O $\quad 5.0794323 .807576-2.085547$

H $\quad 4.6878682 .979528-2.526219$

H $\quad 5.3179843 .627997-1.135874$

O $\quad 1.9335115 .790216-0.256366$

H $\quad 1.5504526 .6277190 .089462$

H $\quad 2.3392625 .794168-1.191123$ 


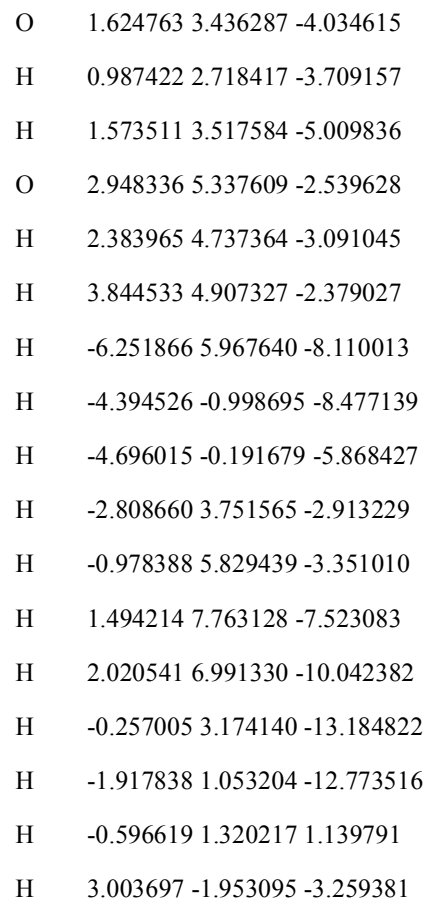

\section{${ }^{2} \mathrm{TS}_{\mathrm{OO}} \mathrm{H}$}




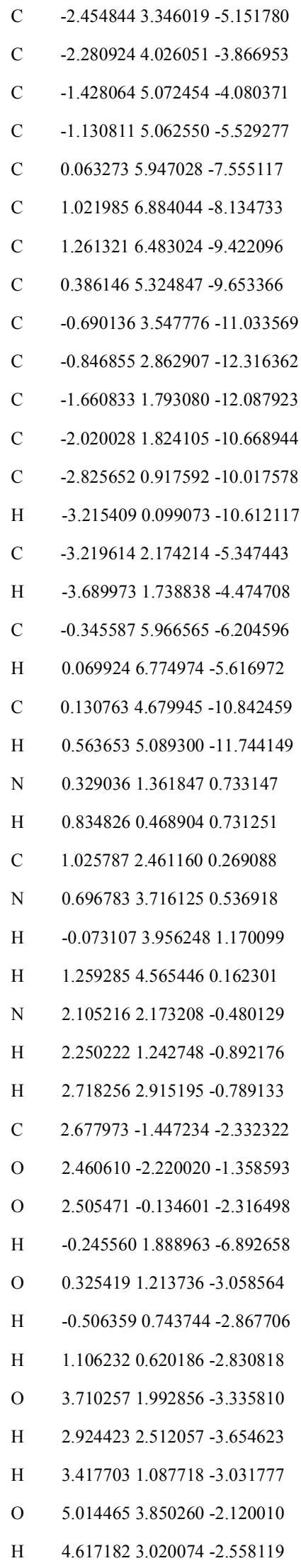

$$
\begin{array}{ll}
\mathrm{H} & 5.2613943 .676146-1.172016 \\
\mathrm{O} & 1.9541125 .797371-0.262865 \\
\mathrm{H} & 1.5498516 .6278050 .076690 \\
\mathrm{H} & 2.2986555 .793737-1.223518 \\
\mathrm{O} & 1.4576613 .378896-3.919172 \\
\mathrm{H} & 0.8525342 .674653-3.523926 \\
\mathrm{H} & 1.3859963 .375753-4.907097 \\
\mathrm{O} & 2.8429955 .342339-2.602458 \\
\mathrm{H} & 2.2638024 .697628-3.092084 \\
\mathrm{H} & 3.7478194 .931621-2.445632 \\
\mathrm{H} & -6.1407825 .921290-8.067512 \\
\mathrm{H} & -4.454228-0.961293-8.520879 \\
\mathrm{H} & -4.712720-0.209941-5.888598 \\
\mathrm{H} & -2.7825843 .702192-2.955076 \\
\mathrm{H} & -0.9522215 .776430-3.397687 \\
\mathrm{H} & 1.4916507 .677853-7.553917 \\
\mathrm{H} & 1.9930736 .938401-10.089356 \\
\mathrm{H} & -0.3907953 .208716-13.243989 \\
\mathrm{H} & -2.0517521 .088261-12.821735 \\
\mathrm{H} & -0.5651931 .3351191 .179927 \\
\mathrm{H} & 3.044777-1.931759-3.237173
\end{array}
$$

\section{${ }^{2} \mathbf{C}_{\mathrm{Ib}} \mathbf{H}$}

$$
\begin{array}{ll}
\mathrm{N} & -4.4703186 .102223-9.527004 \\
\mathrm{H} & -4.7485206 .852156-10.175141 \\
\mathrm{C} & -5.1762895 .586643-8.435899 \\
\mathrm{C} & -3.2680775 .477267-9.603081 \\
\mathrm{H} & -2.5399055 .682462-10.368736 \\
\mathrm{~N} & -3.1630024 .582793-8.604643 \\
\mathrm{C} & -4.3501064 .645759-7.876603 \\
\mathrm{H} & -4.5299983 .998228-7.041446 \\
\mathrm{O} & -0.1430622 .319204-7.742063 \\
\mathrm{O} & 1.7374943 .845032-6.597718 \\
\mathrm{H} & 1.0232783 .272886-7.033382 \\
\mathrm{FE} & -1.6094553 .415719-8.145198 \\
\mathrm{~N} & -2.8397411 .850190-7.776897 \\
\mathrm{~N} & -1.7828953 .940596-6.168316 \\
\mathrm{~N} & -0.3125784 .958797-8.501263 \\
\mathrm{~N} & -1.4394032 .905762-10.081726 \\
\mathrm{C} & -3.2148860 .891241-8.673235 \\
\mathrm{C} & -4.054727-0.124036-8.029760
\end{array}
$$

C $\quad-4.1943710 .243787-6.722796$

C $\quad-3.4270011 .478633-6.555440$

C $\quad-2.4906183 .324749-5.173640$

C $\quad-2.3001783 .996497-3.887134$

C $\quad-1.4119405 .015375-4.093792$

C $\quad-1.1076224 .994634-5.540136$

C $\quad 0.1168915 .848951-7.571095$

C $\quad 1.0590236 .807614-8.152173$

C $\quad 1.2557716 .444784-9.454785$

C $\quad 0.3575465 .300360-9.692773$

C $\quad-0.7343423 .533234-11.063832$

C $\quad-0.8888882 .844981-12.345639$

C $\quad-1.6905151 .767425-12.112615$

C $\quad-2.0456081 .795592-10.691217$

C $\quad-2.8394150 .883284-10.037221$

H $\quad-3.2203380 .059135-10.629640$

C $\quad-3.2612822 .157531-5.371132$

$\mathrm{H} \quad-3.7378881 .726404-4.499461$

C $\quad-0.2491485 .838867-6.203461$

H $\quad 0.2445306 .592116-5.603338$

C $\quad 0.0850974 .670562-10.881894$

H $\quad 0.5061425 .083024-11.787101$

$\mathrm{N} \quad 0.3214631 .3663280 .724651$

H $\quad 0.8276880 .4734830 .723475$

C $\quad 1.0231392 .4676280 .269448$

N $\quad 0.7006953 .7209700 .548985$

$\mathrm{H} \quad-0.0710003 .9575681 .181643$

H $\quad 1.2721734 .5774770 .194379$

N $2.0960972 .179107-0.488902$

H $\quad 2.2388611 .248406-0.902238$

H $\quad 2.7107532 .919884-0.797545$

C $\quad 2.681287-1.432887-2.331160$

O $\quad 2.471268-2.204009-1.354793$

O $\quad 2.501345-0.120867-2.320155$

H $\quad-0.3914081 .727806-7.013291$

O $\quad 0.3897861 .264880-3.186732$

H $\quad-0.4571590 .879177-2.900532$

$\mathrm{H} \quad 1.1448560 .650975-2.923952$

O $\quad 3.7744731 .999995-3.289881$

H $\quad 3.0075052 .542550-3.617927$

H $\quad 3.4594021 .101920-2.991199$ 


$\begin{array}{ll}\mathrm{O} & 5.0855363 .845830-2.056812 \\ \mathrm{H} & 4.6837153 .021467-2.500821 \\ \mathrm{H} & 5.3238403 .660879-1.108504 \\ \mathrm{O} & 1.9667835 .809081-0.191105 \\ \mathrm{H} & 1.5527386 .6386340 .138977 \\ \mathrm{H} & 2.3596905 .811263-1.134592 \\ \mathrm{O} & 1.5971343 .453595-3.883115 \\ \mathrm{H} & 0.9532642 .735752-3.585001 \\ \mathrm{H} & 1.5646223 .626228-4.856705 \\ \mathrm{O} & 2.9363145 .366619-2.489663 \\ \mathrm{H} & 2.3603494 .728916-2.992288 \\ \mathrm{H} & 3.8413574 .951116-2.344235 \\ \mathrm{H} & -6.1485895 .922459-8.075444 \\ \mathrm{H} & -4.469197-0.994813-8.537725 \\ \mathrm{H} & -4.731632-0.238793-5.906381 \\ \mathrm{H} & -2.8189653 .690572-2.978654 \\ \mathrm{H} & -0.9286175 .711812-3.408635 \\ \mathrm{H} & 1.5320317 .592734-7.562329 \\ \mathrm{H} & 1.9782286 .910022-10.125363 \\ \mathrm{H} & -0.4375273 .194340-13.274234 \\ \mathrm{H} & -2.0789591 .061832-12.846997 \\ \mathrm{H} & -0.5679291 .3412561 .181078 \\ \mathrm{H} & 3.048997-1.920358-3.234060\end{array}$

\section{${ }^{2} \mathbf{P H}$}

$\begin{array}{ll}\mathrm{N} & -4.4197936 .102068-9.516635 \\ \mathrm{H} & -4.7002036 .849240-10.166712 \\ \mathrm{C} & -5.1290075 .586861-8.427849 \\ \mathrm{C} & -3.2186735 .474619-9.590802 \\ \mathrm{H} & -2.4942055 .679450-10.358816 \\ \mathrm{~N} & -3.1134274 .577035-8.594003 \\ \mathrm{C} & -4.3045354 .643742-7.869117 \\ \mathrm{H} & -4.4901793 .998186-7.034895 \\ \mathrm{O} & -0.0707232 .312240-7.721830 \\ \mathrm{O} & 1.3121814 .071135-6.179629 \\ \mathrm{H} & 0.9096603 .368613-6.776132 \\ \mathrm{FE} & -1.5550173 .375373-8.150748 \\ \mathrm{~N} & -2.8049041 .836271-7.760965 \\ \mathrm{~N} & -1.6898673 .885467-6.170830 \\ \mathrm{~N} & -0.2269384 .893806-8.524804 \\ \mathrm{~N} & -1.4172142 .887393-10.089934\end{array}$

$\begin{array}{lllll}\mathrm{C} & -3.1640810 .843342-8.669496 & \mathrm{H} & 3.0159982 .468681-3.503685 \\ \mathrm{C} & -4.028223-0.131781-8.023231 & \mathrm{H} & 3.5251771 .035707-2.940256 \\ \mathrm{C} & -4.2066060 .286171-6.729518 & \mathrm{O} & 5.0895323 .836287-2.037180 \\ \mathrm{C} & -3.4394331 .510054-6.586467 & \mathrm{H} & 4.7065322 .998651-2.474085 \\ \mathrm{C} & -2.5801403 .377641-5.184210 & \mathrm{H} & 5.3392973 .658120-1.090427 \\ \mathrm{C} & -2.3446234 .070419-3.920198 & \mathrm{O} & 2.0125475 .805311-0.150773 \\ \mathrm{C} & -1.2794204 .912906-4.112550 & \mathrm{H} & 1.5810886 .6287080 .175242 \\ \mathrm{C} & -0.8975224 .745618-5.518844 & \mathrm{H} & 2.3964285 .820778-1.093899 \\ \mathrm{C} & 0.3132925 .668314-7.584594 & \mathrm{O} & 1.5958673 .331556-3.628029 \\ \mathrm{C} & 1.1250436 .730208-8.147873 & \mathrm{H} & 0.9443772 .620420-3.317855 \\ \mathrm{C} & 1.1837316 .513800-9.500828 & \mathrm{H} & 1.4915713 .529649-4.591285 \\ \mathrm{C} & 0.3013505 .365422-9.762248 & \mathrm{O} & 2.9424195 .364766-2.485991 \\ \mathrm{C} & -0.7791833 .561636-11.098456 & \mathrm{H} & 2.3532614 .655506-2.866759 \\ \mathrm{C} & -0.9452692 .875208-12.364445 & \mathrm{H} & 3.8544794 .963055-2.340692 \\ \mathrm{C} & -1.6903381 .755404-12.105513 & \mathrm{H} & -6.1064065 .919270-8.078190 \\ \mathrm{C} & -1.9953971 .767592-10.685141 & \mathrm{H} & -4.451100-1.006363-8.517547 \\ \mathrm{C} & -2.7719550 .832687-10.006374 & \mathrm{H} & -4.767645-0.172502-5.915318 \\ \mathrm{H} & -3.133726-0.002735-10.597198 & \mathrm{H} & -2.9303853 .856808-3.026151 \\ \mathrm{C} & -3.3478192 .265773-5.390457 & \mathrm{H} & -0.7316975 .568106-3.435218 \\ \mathrm{H} & -3.9015191 .872639-4.545209 & \mathrm{H} & 1.6007157 .478779-7.514277 \\ \mathrm{C} & 0.3754555 .200732-6.166591 & \mathrm{H} & 1.8440397 .056103-10.177560 \\ \mathrm{H} & 0.8705665 .954139-5.555941 & \mathrm{H} & -0.5451693 .241466-13.309877 \\ \mathrm{C} & -0.0187464 .750049-10.938093 & \mathrm{H} & -2.0784661 .044644-12.835062 \\ \mathrm{H} & 0.3301845 .191585-11.860437 & \mathrm{H} & -0.5672651 .3383061 .182811 \\ \mathrm{~N} & 0.3318051 .3586360 .745512 & \mathrm{H} & 3.040121-1.964856-3.219478\end{array}$

H $\quad 0.8287270 .4607370 .743918$

C $\quad 1.0479942 .4548420 .305063$

$\mathrm{N} \quad 0.7245023 .7109060 .573826$

H $\quad-0.0600243 .9534511 .187909$

H $\quad 1.3025734 .5594910 .225221$

N $\quad 2.1414492 .159407-0.421640$

$\mathrm{H} \quad 2.2836621 .229266-0.833401$

H $\quad 2.7731972 .894065-0.709106$

C $\quad 2.686850-1.485533-2.306508$

O $\quad 2.474009-2.261279-1.334494$

O $\quad 2.524753-0.171971-2.282717$

H $\quad-0.3368361 .666691-7.047878$

O $\quad 0.3716551 .180513-3.023989$

$\mathrm{H} \quad-0.4729080 .746386-2.809637$

H $\quad 1.1420870 .568393-2.799175$

O $\quad 3.818951 \quad 1.938088-3.245837$

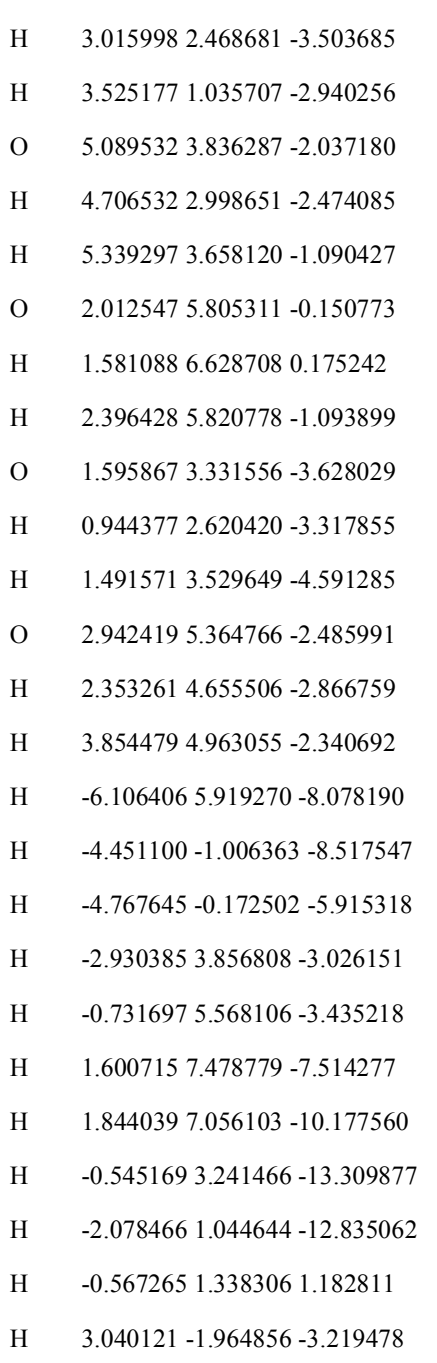

\section{Snapshot 116 ps}

$$
\begin{array}{ll}
{ }^{2} \mathrm{C}_{\mathrm{R}} & \\
\mathrm{N} & -4.2346626 .254491-9.272084 \\
\mathrm{H} & -4.4251827 .080131-9.823223 \\
\mathrm{C} & -5.0581545 .595809-8.351399 \\
\mathrm{C} & -3.0225905 .630911-9.296692 \\
\mathrm{H} & -2.1968515 .958776-9.900831 \\
\mathrm{~N} & -3.0258464 .598737-8.452225 \\
\mathrm{C} & -4.2900744 .563583-7.860881 \\
\mathrm{H} & -4.5371233 .814519-7.130339 \\
\mathrm{O} & -0.1079682 .281678-7.404525 \\
\mathrm{O} & 1.1522322 .870307-6.952397 \\
\mathrm{H} & 1.0649813 .782886-7.298127
\end{array}
$$




\begin{tabular}{|c|c|}
\hline $\mathrm{FE}$ & $-1.4171183 .432678-7.953941$ \\
\hline $\mathrm{N}$ & $-2.6767871 .890769-7.645572$ \\
\hline N & $-1.6970234 .106306-6.067280$ \\
\hline $\mathrm{N}$ & $-0.1150204 .991914-8.310310$ \\
\hline v & $-1.2396902 .880421-9.900133$ \\
\hline C & $-3.0966370 .939658-8.561758$ \\
\hline & $-3.947474-0.050706-7.897174$ \\
\hline $\mathrm{C}$ & $-4.0474300 .322690-6.587772$ \\
\hline $\mathrm{C}$ & $-3.2473891 .540869-6.435148$ \\
\hline $\mathrm{C}$ & $-2.4163943 .463158-5.073886$ \\
\hline$C$ & $-2.3954804 .263008-3.843759$ \\
\hline $\mathrm{C}$ & $-1.6769985 .398112-4.107616$ \\
\hline $\mathrm{C}$ & $-1.2288715 .282685-5.508915$ \\
\hline & $0.1397646 .056994-7.454497$ \\
\hline $\mathrm{C}$ & $1.1344316 .941345-8.047386$ \\
\hline C & $1.5130026 .398384-9.248614$ \\
\hline C & $0.6631075 .220189-9.451102$ \\
\hline $\mathrm{C}$ & $-0.4219163 .465224-10.854708$ \\
\hline$C$ & $-0.5934672 .798111-12.144407$ \\
\hline $\mathrm{C}$ & $-1.5008181 .795848-11.950256$ \\
\hline $\mathrm{C}$ & $-1.8972251 .839344-10.544086$ \\
\hline $\mathrm{C}$ & $-2.7580560 .945442-9.912525$ \\
\hline $\mathrm{H}$ & $-3.1870560 .157707-10.523704$ \\
\hline $\mathrm{C}$ & $-3.1098722 .265345-5.252764$ \\
\hline $\mathrm{H}$ & $-3.6342131 .857579-4.397935$ \\
\hline 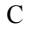 & $-0.4164926 .191168-6.187389$ \\
\hline $\mathrm{H}$ & $-0.1214317 .081497-5.644715$ \\
\hline $\mathrm{C}$ & $0.4675374 .514608-10.633700$ \\
\hline $\mathrm{H}$ & $1.0103564 .846222-11.509087$ \\
\hline $\mathrm{N}$ & 0.8269131 .7294770 .606610 \\
\hline $\mathrm{H}$ & 1.1733530 .8452420 .220235 \\
\hline$C$ & 1.5742162 .8586200 .319035 \\
\hline $\mathrm{N}$ & 1.2648194 .0998610 .705711 \\
\hline $\mathrm{H}$ & 0.4264144 .3191931 .245443 \\
\hline $\mathrm{H}$ & 1.7687704 .8867430 .189215 \\
\hline $\mathrm{N}$ & $2.7079502 .640927-0.366158$ \\
\hline $\mathrm{H}$ & $2.7598561 .850643-1.015509$ \\
\hline $\mathrm{H}$ & $3.3988773 .385849-0.531702$ \\
\hline $\mathrm{C}$ & $2.532389-0.400186-2.129404$ \\
\hline $\mathrm{O}$ & $2.056181-0.621137-0.976001$ \\
\hline D & $2.6997490 .837273-2.5788$ \\
\hline
\end{tabular}

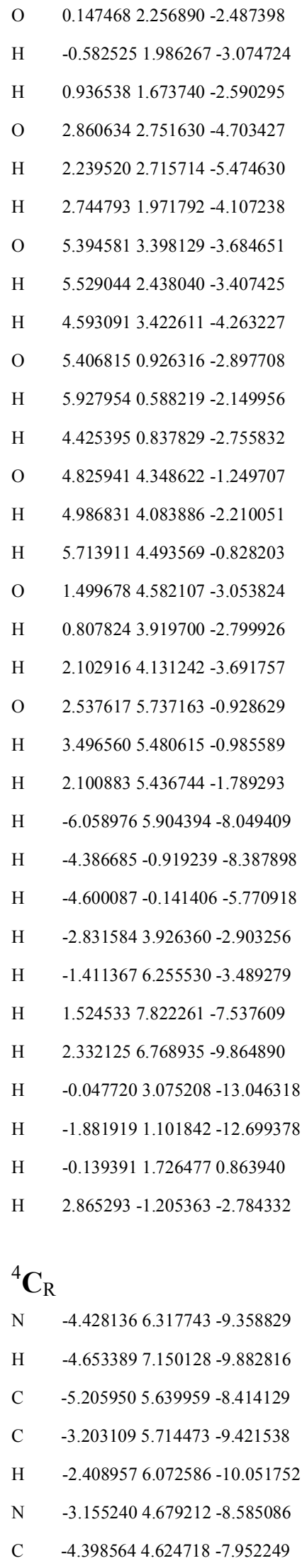

H $\quad-4.6062693 .870604-7.213365$

O $\quad 0.1933432 .230771-7.280390$

O $\quad 1.4565872 .876170-6.941841$

H $\quad 1.3132553 .777841-7.291286$

FE $\quad-1.3180543 .364870-7.977325$

N $\quad-2.5928811 .838977-7.663211$

$\mathrm{N} \quad-1.6784364 .071893-6.090152$

$\mathrm{N} \quad-0.0576094 .953005-8.337354$

$\mathrm{N} \quad-1.1453282 .820636-9.922943$

C $\quad-3.0073090 .884171-8.585012$

C $\quad-3.885224-0.081824-7.930973$

C $\quad-4.0064390 .300352-6.623924$

C $\quad-3.2038771 .508129-6.460437$

C $\quad-2.4078763 .437760-5.099064$

C $\quad-2.3783414 .232736-3.866366$

C $\quad-1.6395895 .355983-4.124364$

C $\quad-1.1914015 .238216-5.527368$

C $\quad 0.1835296 .013461-7.472195$

C $\quad 1.1546086 .919060-8.067665$

C $\quad 1.5302586 .393923-9.279086$

C $\quad 0.7053825 .201112-9.482655$

C $\quad-0.3525243 .430119-10.884559$

C $\quad-0.5377482 .774089-12.176461$

C $\quad-1.4261901 .755493-11.977559$

C $\quad-1.8008161 .777337-10.566109$

C $\quad-2.6560560 .880183-9.933068$

$\mathrm{H} \quad-3.0882040 .095395-10.545510$

C $\quad-3.0988572 .241672-5.280908$

H $\quad-3.6370481 .836019-4.433692$

C $\quad-0.3699946 .139621-6.202147$

$\mathrm{H} \quad-0.0706227 .026735-5.657179$

C $\quad 0.5164384 .495893-10.667397$

H $\quad 1.0450634 .841056-11.545635$

$\mathrm{N} \quad 0.8393481 .7217310 .622185$

$\mathrm{H} \quad 1.1846620 .8367630 .236005$

C $\quad 1.5919402 .8483900 .338813$

$\mathrm{N} \quad 1.2835044 .0904950 .725123$

$\mathrm{H} \quad 0.4411794 .3115191 .257454$

H $\quad 1.7975024 .8767430 .218508$

N $\quad 2.7301062 .627481-0.337498$

H $\quad 2.7878801 .838302-0.988158$ 


\begin{tabular}{|c|c|}
\hline $\mathrm{H}$ & $3.4281753 .368355-0.492854$ \\
\hline $\mathrm{C}$ & $2.570535-0.403128-2.110029$ \\
\hline $\mathrm{O}$ & $2.069118-0.615280-0.965024$ \\
\hline $\mathrm{O}$ & $2.7664770 .829843-2.557760$ \\
\hline $\mathrm{O}$ & $0.2298012 .278185-2.498255$ \\
\hline $\mathrm{H}$ & $-0.4779642 .006686-3.111603$ \\
\hline $\mathrm{H}$ & $1.0261551 .701747-2.587137$ \\
\hline $\mathrm{O}$ & $2.9682402 .811753-4.646706$ \\
\hline $\mathrm{H}$ & $2.3893102 .776381-5.456694$ \\
\hline $\mathrm{H}$ & $2.8406532 .017841-4.074455$ \\
\hline $\mathrm{O}$ & $5.4774023 .406542-3.629104$ \\
\hline $\mathrm{H}$ & $5.6081752 .437751-3.382345$ \\
\hline $\mathrm{H}$ & $4.6744173 .443882-4.207514$ \\
\hline $\mathrm{O}$ & $5.4628990 .902975-2.939700$ \\
\hline $\mathrm{H}$ & $5.9941250 .521019-2.220694$ \\
\hline $\mathrm{H}$ & $4.4828760 .816260-2.783233$ \\
\hline $\mathrm{O}$ & $4.8637074 .319448-1.192630$ \\
\hline $\mathrm{H}$ & $5.0384614 .059468-2.152922$ \\
\hline $\mathrm{H}$ & $5.7493214 .459458-0.764176$ \\
\hline $\mathrm{O}$ & $1.5561804 .628139-3.043675$ \\
\hline $\mathrm{H}$ & $0.8729413 .956375-2.790560$ \\
\hline $\mathrm{H}$ & $2.1751114 .174199-3.666392$ \\
\hline $\mathrm{O}$ & $2.5891885 .732007-0.881578$ \\
\hline $\mathrm{H}$ & $3.5454735 .463394-0.931647$ \\
\hline $\mathrm{H}$ & $2.1584235 .452589-1.751804$ \\
\hline $\mathrm{H}$ & $-6.1930875 .944824-8.066731$ \\
\hline $\mathrm{H}$ & $-4.338742-0.942948-8.421741$ \\
\hline $\mathrm{H}$ & $-4.584364-0.152453-5.818295$ \\
\hline $\mathrm{H}$ & $-2.8239033 .898403-2.929476$ \\
\hline $\mathrm{H}$ & $-1.3582586 .203227-3.498987$ \\
\hline $\mathrm{H}$ & $1.5284177 .806525-7.557039$ \\
\hline $\mathrm{H}$ & $2.3293886 .792451-9.904092$ \\
\hline $\mathrm{H}$ & $-0.0194153 .074059-13.087184$ \\
\hline $\mathrm{H}$ & $-1.8168241 .069772-12.729394$ \\
\hline $\mathrm{H}$ & -0.1264201 .7222740 .881539 \\
\hline $\mathrm{H}$ & $2.900206-1.215899-2.757166$ \\
\hline
\end{tabular}

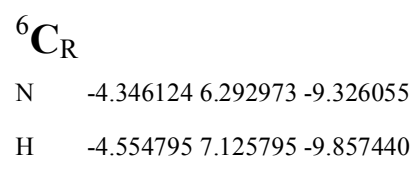

\begin{tabular}{|c|c|c|c|}
\hline $\mathrm{C}$ & $-5.1430985 .624326-8.389552$ & $\mathrm{~N}$ & 1.2641504 .0936930 .714960 \\
\hline $\mathrm{C}$ & $-3.1298275 .674265-9.378280$ & $\mathrm{H}$ & 0.4216804 .3162991 .247206 \\
\hline $\mathrm{H}$ & $-2.3210706 .015706-9.998756$ & $\mathrm{H}$ & 1.7752024 .8787160 .202414 \\
\hline $\mathrm{N}$ & $-3.1037264 .639196-8.539715$ & $\mathrm{~N}$ & $2.7143382 .628798-0.338979$ \\
\hline $\mathrm{C}$ & $-4.3549204 .597494-7.920832$ & $\mathrm{H}$ & $2.7742051 .836299-0.984378$ \\
\hline $\mathrm{H}$ & $-4.5788973 .846360-7.184022$ & $\mathrm{H}$ & $3.4107413 .370550-0.496899$ \\
\hline $\mathrm{O}$ & $0.1655382 .247942-7.242530$ & $\mathrm{C}$ & $2.563373-0.399369-2.124053$ \\
\hline $\mathrm{O}$ & $1.4298432 .878609-6.947153$ & $\mathrm{O}$ & $2.069697-0.612539-0.976104$ \\
\hline $\mathrm{H}$ & $1.2886383 .780878-7.306324$ & $\mathrm{O}$ & $2.7533940 .834882-2.571320$ \\
\hline FE & $-1.3069903 .354073-7.951249$ & $\mathrm{O}$ & $0.2064022 .276374-2.491940$ \\
\hline $\mathrm{N}$ & $-2.6271351 .808454-7.648879$ & $\mathrm{H}$ & $-0.5251331 .997185-3.073060$ \\
\hline $\mathrm{N}$ & $-1.6900894 .107247-6.028338$ & $\mathrm{H}$ & $0.9950591 .691824-2.590683$ \\
\hline $\mathrm{N}$ & $-0.0116014 .982126-8.332847$ & $\mathrm{O}$ & $2.9801092 .777945-4.642138$ \\
\hline $\mathrm{N}$ & $-1.1235472 .803050-9.964510$ & $\mathrm{H}$ & $2.3996572 .748675-5.446244$ \\
\hline $\mathrm{C}$ & $-3.0302150 .862900-8.578893$ & $\mathrm{H}$ & $2.8435431 .989649-4.061365$ \\
\hline $\mathrm{C}$ & $-3.917262-0.097695-7.917928$ & $\mathrm{O}$ & $5.4742443 .393416-3.616923$ \\
\hline $\mathrm{C}$ & $-4.0512750 .296520-6.613326$ & $\mathrm{H}$ & $5.6099172 .431643-3.347930$ \\
\hline $\mathrm{C}$ & $-3.2424081 .505561-6.443892$ & $\mathrm{H}$ & $4.6739793 .415509-4.199975$ \\
\hline $\mathrm{C}$ & $-2.4364823 .469976-5.058985$ & $\mathrm{O}$ & $5.4698420 .905449-2.866648$ \\
\hline $\mathrm{C}$ & $-2.4099074 .275450-3.825029$ & $\mathrm{H}$ & $5.9857590 .547034-2.124573$ \\
\hline $\mathrm{C}$ & $-1.6536885 .389781-4.078787$ & $\mathrm{H}$ & $4.4877330 .820563-2.730953$ \\
\hline $\mathrm{C}$ & $-1.1860515 .265058-5.479933$ & $\mathrm{O}$ & $4.8451684 .328625-1.195688$ \\
\hline $\mathrm{C}$ & $0.2245556 .031341-7.453066$ & $\mathrm{H}$ & $5.0236634 .063095-2.153668$ \\
\hline $\mathrm{C}$ & $1.1868176 .943061-8.062543$ & $\mathrm{H}$ & $5.7292044 .479522-0.767987$ \\
\hline $\mathrm{C}$ & $1.5439926 .431487-9.287533$ & $\mathrm{O}$ & $1.5482524 .608164-3.059916$ \\
\hline $\mathrm{C}$ & $0.7232545 .230965-9.493430$ & $\mathrm{H}$ & $0.8594753 .940620-2.809807$ \\
\hline $\mathrm{C}$ & $-0.3281753 .417661-10.913144$ & $\mathrm{H}$ & $2.1755674 .151001-3.670523$ \\
\hline $\mathrm{C}$ & $-0.5020802 .742983-12.203190$ & $\mathrm{O}$ & $2.5613735 .730442-0.901604$ \\
\hline $\mathrm{C}$ & $-1.3919601 .724135-12.001424$ & $\mathrm{H}$ & $3.5189035 .465660-0.948181$ \\
\hline $\mathrm{C}$ & $-1.7808831 .754078-10.586353$ & $\mathrm{H}$ & $2.1342415 .443904-1.771515$ \\
\hline $\mathrm{C}$ & $-2.6479480 .866976-9.930484$ & $\mathrm{H}$ & $-6.1359915 .930914-8.060562$ \\
\hline $\mathrm{H}$ & $-3.0757030 .077664-10.542350$ & $\mathrm{H}$ & $-4.365868-0.961884-8.407824$ \\
\hline $\mathrm{C}$ & $-3.1324112 .267553-5.268706$ & $\mathrm{H}$ & $-4.631873-0.152237-5.807356$ \\
\hline $\mathrm{H}$ & $-3.6856761 .872715-4.424603$ & $\mathrm{H}$ & $-2.8650703 .944564-2.891536$ \\
\hline $\mathrm{C}$ & $-0.3351866 .142597-6.172021$ & $\mathrm{H}$ & $-1.3735136 .242549-3.460438$ \\
\hline $\mathrm{H}$ & $-0.0167327 .020738-5.620616$ & $\mathrm{H}$ & $1.5641857 .828616-7.551218$ \\
\hline $\mathrm{C}$ & $0.5235354 .506213-10.677175$ & $\mathrm{H}$ & $2.3329076 .839423-9.919409$ \\
\hline $\mathrm{H}$ & $1.0429434 .860079-11.558810$ & $\mathrm{H}$ & $0.0284483 .032584-13.110239$ \\
\hline $\mathrm{N}$ & 0.8244701 .7235920 .622920 & $\mathrm{H}$ & $-1.7883201 .040547-12.752206$ \\
\hline $\mathrm{H}$ & 1.1721910 .8390740 .239366 & $\mathrm{H}$ & -0.1430741 .7216790 .875558 \\
\hline $\mathrm{C}$ & 1.5746672 .8508820 .334905 & $\mathrm{H}$ & $2.891430-1.211056-2.773368$ \\
\hline
\end{tabular}




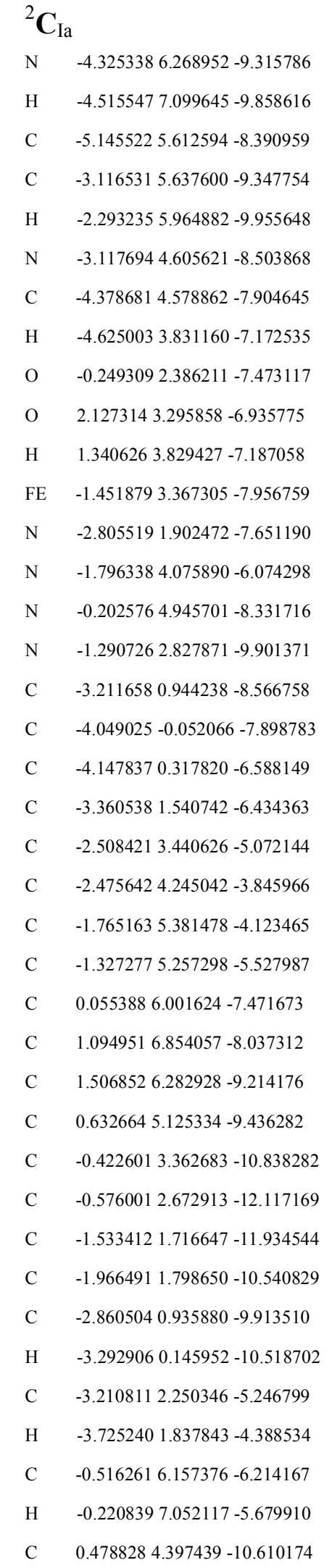

\begin{tabular}{|c|c|c|c|}
\hline $\mathrm{H}$ & $1.0650174 .695905-11.468903$ & $\mathrm{H}$ & $0.0150632 .906473-13.002698$ \\
\hline $\mathrm{N}$ & 0.8139141 .7237830 .639758 & $\mathrm{H}$ & $-1.9319681 .033277-12.684360$ \\
\hline $\mathrm{H}$ & 1.1591910 .8482270 .231901 & $\mathrm{H}$ & -0.1545251 .7203470 .888929 \\
\hline $\mathrm{C}$ & 1.5744422 .8541470 .394590 & $\mathrm{H}$ & $2.847275-1.138365-2.797368$ \\
\hline $\mathrm{N}$ & 1.2682064 .0874820 .805847 & & \\
\hline $\mathrm{H}$ & 0.4112174 .3032281 .318323 & \multicolumn{2}{|c|}{${ }^{2} \mathbf{C}_{\mathrm{Ib}}$} \\
\hline $\mathrm{H}$ & 1.7881724 .8857880 .319348 & $\mathrm{~N}$ & $-4.3146786 .268952-9.305797$ \\
\hline $\mathrm{N}$ & $2.7219452 .644475-0.271343$ & $\mathrm{H}$ & $-4.4989147 .102635-9.846222$ \\
\hline $\mathrm{H}$ & $2.7830761 .870751-0.938437$ & $\mathrm{C}$ & $-5.1407375 .611345-8.386397$ \\
\hline $\mathrm{H}$ & $3.4276093 .385465-0.392916$ & $\mathrm{C}$ & $-3.1085325 .633370-9.336442$ \\
\hline $\mathrm{C}$ & $2.531741-0.334145-2.132736$ & $\mathrm{H}$ & $-2.2805225 .959364-9.938404$ \\
\hline $\mathrm{O}$ & $2.036697-0.565468-0.987811$ & $\mathrm{~N}$ & $-3.1172924 .597483-8.497545$ \\
\hline $\mathrm{O}$ & $2.7425410 .904996-2.554074$ & $\mathrm{C}$ & $-4.3798594 .572328-7.902284$ \\
\hline $\mathrm{O}$ & $0.2284602 .370976-2.470016$ & $\mathrm{H}$ & $-4.6312153 .822205-7.174297$ \\
\hline $\mathrm{H}$ & $-0.4899432 .097125-3.069393$ & $\mathrm{O}$ & $-0.2080732 .428930-7.473927$ \\
\hline $\mathrm{H}$ & $1.0235941 .793418-2.566686$ & $\mathrm{O}$ & $2.0947513 .452135-6.753474$ \\
\hline $\mathrm{O}$ & $3.1397373 .020379-4.425282$ & $\mathrm{H}$ & $1.1746753 .101106-6.985712$ \\
\hline $\mathrm{H}$ & $2.7079863 .078098-5.319422$ & $\mathrm{FE}$ & $-1.4636933 .357579-7.959358$ \\
\hline $\mathrm{H}$ & $2.9316402 .180110-3.951153$ & $\mathrm{~N}$ & $-2.8072471 .890182-7.639953$ \\
\hline $\mathrm{O}$ & $5.6015723 .445911-3.406744$ & $\mathrm{~N}$ & $-1.7966424 .062463-6.069289$ \\
\hline $\mathrm{H}$ & $5.7079672 .477413-3.156814$ & $\mathrm{~N}$ & $-0.2144064 .935014-8.347449$ \\
\hline $\mathrm{H}$ & $4.7910643 .498427-3.981264$ & $\mathrm{~N}$ & $-1.3058002 .809222-9.898468$ \\
\hline $\mathrm{O}$ & $5.4857030 .922880-2.745852$ & $\mathrm{C}$ & $-3.2167990 .928546-8.551215$ \\
\hline $\mathrm{H}$ & $5.9478340 .531234-1.985007$ & $\mathrm{C}$ & $-4.059874-0.061828-7.880606$ \\
\hline $\mathrm{H}$ & $4.4966330 .864192-2.664252$ & $\mathrm{C}$ & $-4.1631590 .316293-6.573175$ \\
\hline $\mathrm{O}$ & $4.8525234 .374619-1.031104$ & $\mathrm{C}$ & $-3.3717391 .537500-6.423292$ \\
\hline $\mathrm{H}$ & $5.0753814 .100596-1.979798$ & $\mathrm{C}$ & $-2.5153563 .436598-5.066439$ \\
\hline $\mathrm{H}$ & $5.7268074 .539537-0.589401$ & $\mathrm{C}$ & $-2.4665214 .236726-3.837530$ \\
\hline $\mathrm{O}$ & $1.5263384 .754799-2.942637$ & $\mathrm{C}$ & $-1.7320895 .358310-4.111996$ \\
\hline $\mathrm{H}$ & $0.8511344 .063276-2.722779$ & $\mathrm{C}$ & $-1.2966055 .228418-5.517264$ \\
\hline $\mathrm{H}$ & $2.2076704 .312990-3.507753$ & $\mathrm{C}$ & $0.1000215 .946716-7.459770$ \\
\hline $\mathrm{O}$ & $2.5654265 .774165-0.743734$ & $\mathrm{C}$ & $1.1536346 .790490-8.022145$ \\
\hline $\mathrm{H}$ & $3.5200495 .500385-0.805632$ & $\mathrm{C}$ & $1.5363966 .231875-9.211877$ \\
\hline $\mathrm{H}$ & $2.1234615 .531271-1.619721$ & $\mathrm{C}$ & $0.6260935 .099436-9.441145$ \\
\hline $\mathrm{H}$ & $-6.1392145 .928363-8.073262$ & $\mathrm{C}$ & $-0.4373593 .340238-10.839296$ \\
\hline $\mathrm{H}$ & $-4.474915-0.928641-8.386944$ & $\mathrm{C}$ & $-0.5877262 .638870-12.112913$ \\
\hline $\mathrm{H}$ & $-4.692224-0.152417-5.769256$ & $\mathrm{C}$ & $-1.5483191 .687225-11.925515$ \\
\hline $\mathrm{H}$ & $-2.8973133 .909031-2.898677$ & $\mathrm{C}$ & $-1.9802911 .776723-10.531509$ \\
\hline $\mathrm{H}$ & $-1.4991076 .243377-3.511574$ & $\mathrm{C}$ & $-2.8703310 .913948-9.898491$ \\
\hline $\mathrm{H}$ & $1.4898347 .729877-7.522460$ & $\mathrm{H}$ & $-3.3032490 .120105-10.497465$ \\
\hline $\mathrm{H}$ & $2.3623386 .620959-9.798936$ & $\mathrm{C}$ & $-3.2277012 .251808-5.238821$ \\
\hline
\end{tabular}




\begin{tabular}{|c|c|}
\hline . & $-3.7472861 .846405-4.380509$ \\
\hline$C$ & $-0.4553216 .104288-6.194992$ \\
\hline$H$ & $-0.1135896 .972808-5.645721$ \\
\hline 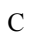 & $0.4634244 .375221-10.617837$ \\
\hline I & $1.0493514 .669746-11.477895$ \\
\hline 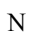 & 0.8256891 .7424920 .632836 \\
\hline $\mathrm{H}$ & 1.1717830 .8696890 .218001 \\
\hline 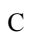 & 1.5945122 .8721070 .411617 \\
\hline 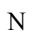 & 1.2863234 .1035890 .827059 \\
\hline $\mathrm{H}$ & 0.4212654 .3189451 .325823 \\
\hline $\mathrm{H}$ & 1.8223654 .9024180 .359973 \\
\hline $\mathrm{N}$ & $2.7532292 .663256-0.235283$ \\
\hline $\mathrm{H}$ & $2.8240311 .892715-0.904679$ \\
\hline $\mathrm{H}$ & $3.4625923 .402593-0.342590$ \\
\hline $\mathrm{C}$ & $2.552383-0.292006-2.124916$ \\
\hline $\mathrm{O}$ & $2.035791-0.526968-0.989809$ \\
\hline $\mathrm{O}$ & $2.7885850 .946626-2.532008$ \\
\hline $\mathrm{O}$ & $0.3199542 .489769-2.488802$ \\
\hline $\mathrm{H}$ & $-0.3922112 .237037-3.104491$ \\
\hline $\mathrm{H}$ & $1.1004551 .889640-2.566595$ \\
\hline $\mathrm{O}$ & $3.2474643 .101654-4.364494$ \\
\hline $\mathrm{H}$ & $2.8013593 .171653-5.257250$ \\
\hline $\mathrm{H}$ & $3.0192522 .269181-3.888235$ \\
\hline $\mathrm{O}$ & $5.6855983 .476740-3.339551$ \\
\hline $\mathrm{H}$ & $5.7796252 .503875-3.105019$ \\
\hline $\mathrm{H}$ & $4.8781613 .544929-3.919510$ \\
\hline $\mathrm{O}$ & $5.5349340 .938067-2.712231$ \\
\hline $\mathrm{H}$ & $5.9894860 .550616-1.944331$ \\
\hline $\mathrm{H}$ & $4.5451980 .889390-2.633725$ \\
\hline $\mathrm{O}$ & $4.9054034 .381077-0.965883$ \\
\hline$H$ & $5.1359744 .113808-1.915188$ \\
\hline 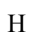 & $5.7785094 .547504-0.522378$ \\
\hline ) & $1.6154394 .876585-2.934983$ \\
\hline$H$ & $0.9469724 .175015-2.722601$ \\
\hline $\mathrm{H}$ & $2.3123364 .434945-3.481493$ \\
\hline $\mathrm{O}$ & $2.6329945 .797307-0.674823$ \\
\hline$H$ & $3.5848045 .511781-0.732631$ \\
\hline$H$ & $2.2021695 .598175-1.566165$ \\
\hline 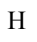 & $-6.1348835 .928160-8.071168$ \\
\hline$H$ & $-4.488149-0.938587-8.366343$ \\
\hline & $4.712815-0.147158-5.75393$ \\
\hline
\end{tabular}

$$
\begin{array}{ll}
\mathrm{H} & -2.8907613 .905140-2.889829 \\
\mathrm{H} & -1.4537456 .214733-3.497886 \\
\mathrm{H} & 1.5580387 .658497-7.501483 \\
\mathrm{H} & 2.3958956 .558718-9.797113 \\
\mathrm{H} & 0.0136612 .857330-12.995338 \\
\mathrm{H} & -1.9546441 .006545-12.673606 \\
\mathrm{H} & -0.1435191 .7391030 .879001 \\
\mathrm{H} & 2.865911-1.094533-2.792536
\end{array}
$$

\section{${ }^{2} \mathbf{C}_{\mathrm{R}} \mathbf{H}$}

$\mathrm{N} \quad-4.3482706 .220684-9.346383$

H $\quad-4.5488547 .034251-9.914812$

C $\quad-5.1482925 .593101-8.381823$

C $\quad-3.1403455 .610618-9.388662$

H $\quad-2.3392295 .912653-10.036548$

$\mathrm{N} \quad-3.1218894 .612615-8.495968$

C $\quad-4.3686434 .587895-7.865669$

$\mathrm{H} \quad-4.5955583 .867970-7.100959$

O $\quad 0.2023492 .337638-7.570086$

O $\quad 1.3415503 .021334-6.971632$

H $\quad 1.0770623 .942955-7.198152$

FE $\quad-1.5949173 .536030-8.030209$

N $\quad-2.7158831 .916803-7.659092$

N $\quad-1.7897504 .112668-6.105982$

N $\quad-0.2176835 .008634-8.331716$

N $\quad-1.2885242 .904581-9.909510$

C $\quad-3.1480490 .965936-8.587052$

C $\quad-4.023068-0.003221-7.935894$

C $\quad-4.1641050 .386251-6.633140$

C $\quad-3.3482941 .581064-6.460216$

C $\quad-2.5257903 .487374-5.101609$

C $\quad-2.5194384 .295462-3.887281$

C $\quad-1.8170115 .438681-4.159303$

C $\quad-1.3553105 .315157-5.551603$

C $\quad 0.0230146 .088720-7.479534$

C $\quad 1.0446996 .950639-8.049477$

C $\quad 1.4546826 .390529-9.233505$

C $\quad 0.6021375 .225573-9.454023$

C $\quad-0.4416283 .466280-10.861972$

C $\quad-0.5881632 .781633-12.138519$

C $\quad-1.5146451 .794586-11.952500$
C $\quad-1.9412171 .858333-10.559917$

C $\quad-2.8044250 .966035-9.933841$

H $\quad-3.2328290 .179539-10.545553$

C $\quad-3.2322382 .302815-5.278421$

H $\quad-3.7915331 .918715-4.434893$

C $\quad-0.5572266 .233023-6.226032$

H $\quad-0.2923157 .135425-5.690763$

C $\quad 0.4433244 .512458-10.634202$

H $\quad 1.0155024 .831108-11.494456$

$\mathrm{N} \quad 0.7349171 .6846791 .200818$

H $\quad 1.1835840 .7912121 .340849$

C $\quad 1.5038992 .7303490 .700056$

$\mathrm{N} \quad 1.1436364 .0054940 .779890$

H $\quad 0.2940954 .3057641 .268161$

$\mathrm{H} \quad 1.6507074 .7187520 .155310$

$\mathrm{N} \quad 2.6820962 .3764080 .167741$

H $\quad 2.8147521 .435461-0.225372$

H $\quad 3.3904193 .083443-0.098533$

C $\quad 2.698778-0.048842-2.369495$

O $\quad 3.168004-0.142337-1.167036$

O $\quad 1.9968020 .948801-2.775793$

H $\quad 0.1808571 .498870-7.047722$

O $\quad-0.1695142 .292470-2.038534$

H $\quad-0.9638811 .894829-2.442270$

H $\quad 0.6303301 .713299-2.186345$

O $\quad 2.8869462 .679609-4.522404$

H $\quad 2.4604492 .746624-5.399652$

H $\quad 2.4845921 .945015-3.964330$

O $\quad 5.2558512 .810036-3.145584$

H $\quad 5.4059131 .878949-2.797937$

H $\quad 4.5034532 .853029-3.788981$

O $\quad 5.7029240 .448867-2.044324$

H $\quad 6.5594320 .320738-1.598425$

H $4.9423090 .121286-1.503451$

O $\quad 4.6593774 .071826-0.910865$

H $4.8606653 .644409-1.807892$

H $\quad 5.5183824 .401876-0.534233$

O $\quad 1.2113754 .457504-3.070369$

H $\quad 0.5532543 .809231-2.701489$

H $\quad 1.8859093 .967321-3.597415$

O $\quad 2.3969145 .517824-0.946870$ 


$$
\begin{array}{ll}
\mathrm{H} & 3.3393275 .193252-0.963533 \\
\mathrm{H} & 1.9382705 .272311-1.806995 \\
\mathrm{H} & -6.1435875 .906869-8.067172 \\
\mathrm{H} & -4.453739-0.877052-8.424778 \\
\mathrm{H} & -4.759127-0.060790-5.836792 \\
\mathrm{H} & -2.9377723 .953228-2.940741 \\
\mathrm{H} & -1.5733186 .302034-3.540189 \\
\mathrm{H} & 1.4429117 .826779-7.537743 \\
\mathrm{H} & 2.2925886 .748228-9.831862 \\
\mathrm{H} & -0.0173873 .035114-13.031844 \\
\mathrm{H} & -1.8951491 .101510-12.702786 \\
\mathrm{H} & -0.2597001 .6706651 .303345 \\
\mathrm{H} & 2.957111-0.850604-3.061238
\end{array}
$$

\section{${ }^{4} \mathbf{C}_{\mathrm{R}} \mathbf{H}$}

$$
\begin{aligned}
& \text { N } \quad-4.5835006 .272681-9.423493 \\
& \text { H } \quad-4.8238517 .094515-9.958783 \\
& \text { C } \quad-5.3441685 .616812-8.449947 \\
& \text { C } \quad-3.3608965 .683787-9.497912 \\
& \text { H } \quad-2.5838176 .021458-10.158735 \\
& \text { N } \quad-3.3009254 .673323-8.626553 \\
& \text { C } \quad-4.5316494 .619495-7.970065 \\
& \text { H } \quad-4.7274103 .882766-7.211243 \\
& \text { O } \quad 0.5064402 .297308-7.463196 \\
& \text { O } \quad 1.6255513 .048158-6.915752 \\
& \text { H } \quad 1.2650663 .950188-7.047966 \\
& \text { FE } \quad-1.6025483 .531774-8.061056 \\
& \text { N } \quad-2.6891311 .889340-7.675668 \\
& \mathrm{~N} \quad-1.8049264 .120270-6.134058 \\
& \text { N } \quad-0.1957964 .978880-8.338589 \\
& \mathrm{~N} \quad-1.2343572 .843162-9.906993 \\
& \text { C } \quad-3.1038230 .917064-8.594519 \\
& \text { C } \quad-3.983905-0.039240-7.940268 \\
& \text { C } \quad-4.1360330 .366219-6.642435 \\
& \text { C } \quad-3.3305751 .566449-6.476807 \\
& \text { C } \quad-2.5425843 .493873-5.131261 \\
& \text { C } \quad-2.5339474 .299737-3.916188 \\
& \text { C } \quad-1.8229575 .439866-4.183336 \\
& \text { C } \quad-1.3569795 .316360-5.570964 \\
& \text { C } \quad 0.0489606 .057704-7.485931 \\
& \text { C } \quad 1.0797756 .909503-8.051207
\end{aligned}
$$

\begin{tabular}{|c|c|}
\hline $\mathrm{N}$ & $-4.6037286 .281618-9.431094$ \\
\hline $\mathrm{H}$ & $-4.8451487 .103689-9.965709$ \\
\hline $\mathrm{C}$ & $-5.3630205 .625485-8.455574$ \\
\hline $\mathrm{C}$ & $-3.3823295 .697541-9.509005$ \\
\hline $\mathrm{H}$ & $-2.6055876 .034274-10.170644$ \\
\hline N & $-3.3216104 .686103-8.635896$ \\
\hline $\mathrm{C}$ & $-4.5518674 .628640-7.975951$ \\
\hline $\mathrm{H}$ & $-4.7438013 .894404-7.213528$ \\
\hline $\mathrm{O}$ & $0.4852952 .305211-7.468285$ \\
\hline $\mathrm{O}$ & $1.5988593 .051308-6.900931$ \\
\hline $\mathrm{H}$ & $1.2411663 .955033-7.029511$ \\
\hline $\mathrm{FE}$ & $-1.6526233 .567368-8.083125$ \\
\hline N & $-2.7040761 .855533-7.669459$ \\
\hline $\mathrm{N}$ & $-1.7899814 .129610-6.087732$ \\
\hline N & $-0.1626355 .010704-8.346076$ \\
\hline N & $-1.1960742 .809541-9.943321$ \\
\hline $\mathrm{C}$ & $-3.1046400 .885160-8.593647$ \\
\hline $\mathrm{C}$ & $-3.991036-0.066423-7.933556$ \\
\hline $\mathrm{C}$ & $-4.1457960 .342906-6.635274$ \\
\hline $\mathrm{C}$ & $-3.3400821 .546380-6.465826$ \\
\hline $\mathrm{C}$ & $-2.5339363 .495797-5.098085$ \\
\hline
\end{tabular}

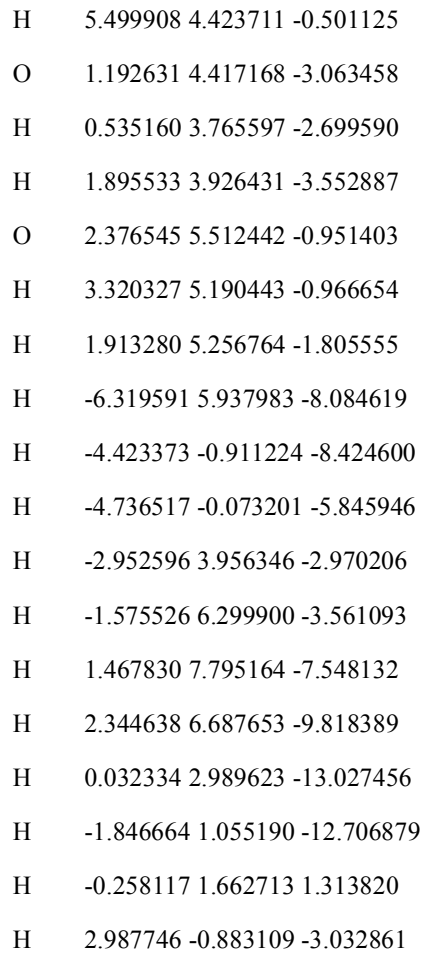

\section{${ }^{6} \mathbf{C}_{\mathrm{R}} \mathrm{H}$}




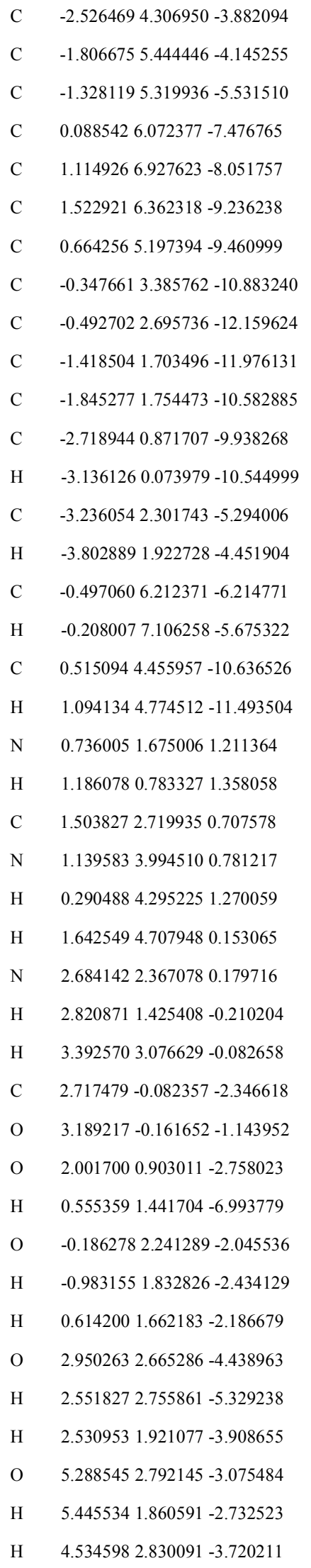

$$
\begin{aligned}
& \text { O } \quad 5.7298510 .417342-1.999798 \\
& \text { H } \quad 6.5812780 .285262-1.545118 \\
& \text { H } \quad 4.9618460 .091963-1.467666 \\
& \text { O } \quad 4.6468924 .079059-0.881133 \\
& \text { H } \quad 4.8685083 .633584-1.766848 \\
& \text { H } \quad 5.4987704 .421710-0.500181 \\
& \text { O } \quad 1.1972064 .412465-3.063053 \\
& \text { H } \quad 0.5376263 .762145-2.700422 \\
& \text { H } \quad 1.8985953 .920983-3.553686 \\
& \text { O } \quad 2.3762875 .509960-0.952652 \\
& \text { H } \quad 3.3201165 .187959-0.966701 \\
& \text { H } \quad 1.9144045 .252079-1.807223 \\
& \text { H } \quad-6.3366285 .947499-8.086166 \\
& \text { H } \quad-4.435794-0.934662-8.419786 \\
& \text { H } \quad-4.748002-0.094844-5.839165 \\
& \text { H } \quad-2.9545203 .965717-2.939545 \\
& \text { H } \quad-1.5658036 .309922-3.528005 \\
& \mathrm{H} \quad 1.5037257 .812350-7.547615 \\
& \text { H } \quad 2.3678466 .713110-9.828786 \\
& \text { H } \quad 0.0664912 .961544-13.056684 \\
& \text { H } \quad-1.8183981 .029244-12.733452 \\
& \mathrm{H} \quad-0.2586101 .6607561 .313873 \\
& \text { H } \quad 2.986347-0.886487-3.031564
\end{aligned}
$$

\section{${ }^{2} \mathrm{TS}_{\mathbf{O O}} \mathrm{H}$}

$$
\begin{array}{ll}
\mathrm{N} & -4.3220996 .213001-9.327178 \\
\mathrm{H} & -4.5129957 .032503-9.890940 \\
\mathrm{C} & -5.1315715 .583846-8.373203 \\
\mathrm{C} & -3.1180905 .591529-9.366156 \\
\mathrm{H} & -2.3140825 .893171-10.010220 \\
\mathrm{~N} & -3.1070224 .584575-8.484115 \\
\mathrm{C} & -4.3600644 .567252-7.864221 \\
\mathrm{H} & -4.6028253 .841457-7.110127 \\
\mathrm{O} & 0.0132732 .366863-7.659485 \\
\mathrm{O} & 1.5467932 .951069-6.918411 \\
\mathrm{H} & 1.3079863 .880276-7.092548 \\
\mathrm{FE} & -1.5458923 .487228-8.010828 \\
\mathrm{~N} & -2.7287011 .900781-7.650359 \\
\mathrm{~N} & -1.7926484 .096095-6.084002 \\
\mathrm{~N} & -0.2310585 .015985-8.345509 \\
\mathrm{~N} & -1.2954342 .891823-9.906886
\end{array}
$$

C $\quad-3.1605940 .953760-8.570641$

C $\quad-4.036349-0.019380-7.922104$

C $\quad-4.1745180 .365758-6.618844$

C $\quad-3.3562671 .561502-6.445782$

C $\quad-2.5306613 .484244-5.089211$

C $\quad-2.5416264 .296428-3.877950$

C $\quad-1.8269215 .433845-4.148071$

C $\quad-1.3508785 .294363-5.533990$

C $\quad 0.0334346 .061960-7.479790$

C $\quad 1.0725466 .918922-8.031996$

C $\quad 1.4833946 .357507-9.215404$

C $\quad 0.6118305 .204066-9.438062$

C $\quad-0.4267013 .430359-10.850279$

C $\quad-0.5628062 .734997-12.124410$

C $\quad-1.5067261 .764722-11.944802$

C $\quad-1.9481291 .844386-10.556101$

C $\quad-2.8158850 .961798-9.923274$

H $\quad-3.2475570 .175609-10.533801$

C $\quad-3.2373182 .288922-5.271193$

H $\quad-3.7957461 .906144-4.426072$

C $\quad-0.5386836 .196561-6.213640$

H $\quad-0.2532237 .090181-5.673329$

C $\quad 0.4607134 .473136-10.614996$

H $\quad 1.0495684 .776071-11.469880$

$\mathrm{N} \quad 0.7330271 .6673491 .209584$

H $\quad 1.1840270 .7742141 .344276$

C $\quad 1.4964752 .7137400 .701086$

N $\quad 1.1316023 .9879810 .778605$

$\mathrm{H} \quad 0.2836624 .2870981 .270371$

H $\quad 1.6267174 .7032580 .145612$

$\mathrm{N} \quad 2.6727452 .3620500 .164552$

H $\quad 2.8057331 .419015-0.224856$

H $\quad 3.3758223 .072844-0.109670$

C $\quad 2.654711-0.061716-2.354461$

O $3.146864-0.162761-1.159302$

O $\quad 1.9164430 .918005-2.730543$

H $\quad-0.0492921 .571156-7.086217$

O $\quad-0.2575792 .251241-2.009226$

H $\quad-1.0490451 .820754-2.385368$

H $\quad 0.5523261 .685058-2.154474$

O $\quad 2.7936652 .686957-4.466294$ 


\begin{tabular}{|c|c|}
\hline $\mathrm{H}$ & $2.3788842 .745728-5.361445$ \\
\hline $\mathrm{H}$ & $2.4006781 .936765-3.929415$ \\
\hline $\mathrm{O}$ & $5.1764612 .803636-3.145486$ \\
\hline $\mathrm{H}$ & $5.3374171 .876986-2.792088$ \\
\hline $\mathrm{H}$ & $4.4044212 .840660-3.770598$ \\
\hline $\mathrm{O}$ & $5.6634830 .459351-2.022460$ \\
\hline $\mathrm{H}$ & $6.5186020 .343304-1.571192$ \\
\hline $\mathrm{H}$ & $4.9019630 .112375-1.493499$ \\
\hline $\mathrm{O}$ & $4.6104264 .082022-0.924081$ \\
\hline $\mathrm{H}$ & $4.8085243 .646659-1.820546$ \\
\hline $\mathrm{H}$ & $5.4696804 .410540-0.547339$ \\
\hline $\mathrm{O}$ & $1.1202414 .430401-3.054491$ \\
\hline $\mathrm{H}$ & $0.4642883 .783694-2.682146$ \\
\hline $\mathrm{H}$ & $1.7935393 .935113-3.584675$ \\
\hline $\mathrm{O}$ & $2.3407745 .512177-0.966365$ \\
\hline $\mathrm{H}$ & $3.2850305 .192352-0.989613$ \\
\hline $\mathrm{H}$ & $1.8689115 .249816-1.815511$ \\
\hline $\mathrm{H}$ & $-6.1277015 .900445-8.064082$ \\
\hline $\mathrm{H}$ & $-4.468692-0.890338-8.414626$ \\
\hline $\mathrm{H}$ & $-4.768612-0.081050-5.821673$ \\
\hline $\mathrm{H}$ & $-2.9741893 .962929-2.934693$ \\
\hline $\mathrm{H}$ & $-1.5858656 .300785-3.532950$ \\
\hline $\mathrm{H}$ & $1.4720827 .790841-7.514122$ \\
\hline $\mathrm{H}$ & $2.3307806 .705051-9.806349$ \\
\hline $\mathrm{H}$ & $0.0250592 .974326-13.010527$ \\
\hline $\mathrm{H}$ & $-1.894011 \quad 1.076825-12.696383$ \\
\hline $\mathrm{H}$ & -0.2611201 .6508441 .316221 \\
\hline $\mathrm{H}$ & $2.927303-0.842888-3.064060$ \\
\hline
\end{tabular}

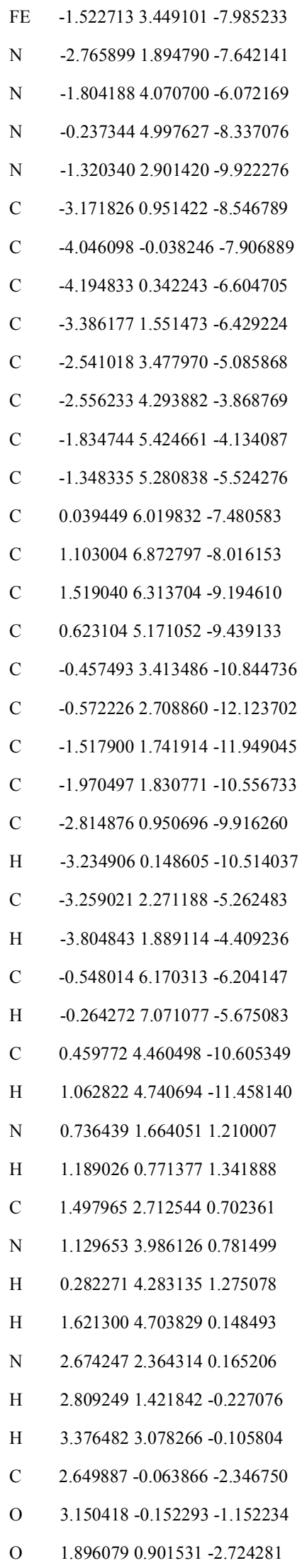

H $\quad-0.1003821 .663024-7.036403$

O $\quad-0.2856042 .222851-2.007867$

H $\quad-1.0749221 .768902-2.361092$

H $\quad 0.5324101 .668110-2.156076$

O $\quad 2.8035982 .751164-4.388544$

H $\quad 2.4719572 .831516-5.324145$

H $\quad 2.4029601 .970089-3.912124$

O $\quad 5.1816402 .804909-3.104676$

H $\quad 5.3459741 .875747-2.760975$

H $\quad 4.3929892 .851317-3.713467$

O $\quad 5.6673830 .444017-2.008001$

H $\quad 6.5187610 .320056-1.551985$

H $\quad 4.8987070 .102575-1.484471$

O $\quad 4.5972564 .097939-0.908971$

$\mathrm{H} \quad 4.8084783 .648475-1.798148$

H $\quad 5.4526134 .431923-0.528947$

O $\quad 1.0687974 .432840-3.037319$

H $\quad 0.4223613 .777944-2.664059$

H $\quad 1.7587923 .941358-3.555102$

O $\quad 2.3227895 .516959-0.967611$

H $\quad 3.2669945 .195617-0.996117$

H $\quad 1.8386325 .250589-1.808732$

H $\quad-6.1406625 .907659-8.068366$

H $\quad-4.475288-0.907484-8.405173$

H $\quad-4.784955-0.109465-5.807345$

H $\quad-2.9936813 .962190-2.927129$

H $\quad-1.5888576 .291431-3.520642$

H $\quad 1.5024647 .737583-7.486395$

H $\quad 2.3795646 .646598-9.774909$

H $\quad 0.0279242 .942464-13.003086$

H $\quad-1.9065751 .054576-12.700420$

$\mathrm{H} \quad-0.2573591 .6471231 .319783$

H $\quad 2.931912-0.846248-3.051312$

\section{${ }^{2} \mathbf{C}_{\text {Ib }} \mathbf{H}$}

$\begin{array}{ll}\mathrm{N} & -4.3333136 .216334-9.331412 \\ \mathrm{H} & -4.5031337 .046055-9.887329 \\ \mathrm{C} & -5.1667275 .582797-8.402865 \\ \mathrm{C} & -3.1345215 .578025-9.353277 \\ \mathrm{H} & -2.3161425 .878795-9.979622 \\ \mathrm{~N} & -3.1502274 .557450-8.488786\end{array}$ 


\begin{tabular}{|c|c|}
\hline$C$ & $-4.4169604 .547411-7.897861$ \\
\hline $\mathrm{H}$ & $-4.6842873 .813689-7.159709$ \\
\hline $\mathrm{O}$ & $-0.0752792 .461830-7.465689$ \\
\hline $\mathrm{O}$ & $2.2270653 .413750-6.793754$ \\
\hline $\mathrm{H}$ & $1.2952783 .057186-7.045556$ \\
\hline FE & $-1.5718343 .417378-7.957455$ \\
\hline $\mathrm{N}$ & $-2.8166351 .860560-7.605912$ \\
\hline $\mathrm{N}$ & $-1.8910264 .080104-6.063853$ \\
\hline N & $-0.2647834 .949012-8.304987$ \\
\hline $\mathrm{N}$ & $-1.3394472 .827795-9.864861$ \\
\hline $\mathrm{C}$ & $-3.2288390 .911502-8.502514$ \\
\hline $\mathrm{C}$ & $-4.108590-0.068156-7.856707$ \\
\hline $\mathrm{C}$ & $-4.2569980 .325239-6.558383$ \\
\hline $\mathrm{C}$ & $-3.4469171 .536195-6.394018$ \\
\hline $\mathrm{C}$ & $-2.6156243 .484749-5.071283$ \\
\hline $\mathrm{C}$ & $-2.6071284 .296884-3.850189$ \\
\hline $\mathrm{C}$ & $-1.8839345 .425120-4.121910$ \\
\hline $\mathrm{C}$ & $-1.4127355 .281042-5.515842$ \\
\hline $\mathrm{C}$ & $0.0231605 .965447-7.454901$ \\
\hline $\mathrm{C}$ & $1.1196806 .791848-7.974271$ \\
\hline $\mathrm{C}$ & $1.5492666 .210275-9.134183$ \\
\hline $\mathrm{C}$ & $0.6295575 .083911-9.384778$ \\
\hline $\mathrm{C}$ & $-0.4549693 .320876-10.780789$ \\
\hline $\mathrm{C}$ & $-0.5692502 .611593-12.055105$ \\
\hline $\mathrm{C}$ & $-1.5452461 .671850-11.891448$ \\
\hline $\mathrm{C}$ & $-2.0082821 .770800-10.505377$ \\
\hline $\mathrm{C}$ & $-2.8684630 .902142-9.869966$ \\
\hline $\mathrm{H}$ & $-3.2954730 .102666-10.465527$ \\
\hline $\mathrm{C}$ & $-3.3315302 .275965-5.237590$ \\
\hline $\mathrm{H}$ & $-3.8835161 .906410-4.382430$ \\
\hline $\mathrm{C}$ & $-0.5840736 .146967-6.191323$ \\
\hline $\mathrm{H}$ & $-0.2816587 .043242-5.6644$ \\
\hline $\mathrm{C}$ & $0.4778954 .356460-10.540090$ \\
\hline $\mathrm{H}$ & $1.1008994 .612074-11.386130$ \\
\hline $\mathrm{N}$ & 0.7302321 .6700901 .207862 \\
\hline $\mathrm{H}$ & 1.1770710 .7746731 .341193 \\
\hline $\mathrm{C}$ & 1.5045312 .7195520 .722964 \\
\hline $\mathrm{N}$ & 1.1443983 .9943400 .809371 \\
\hline $\mathrm{H}$ & 0.2909664 .2921141 .292143 \\
\hline $\mathrm{H}$ & 1.6500294 .7129880 .187465 \\
\hline N & 6873282.3689580 .1995 \\
\hline
\end{tabular}

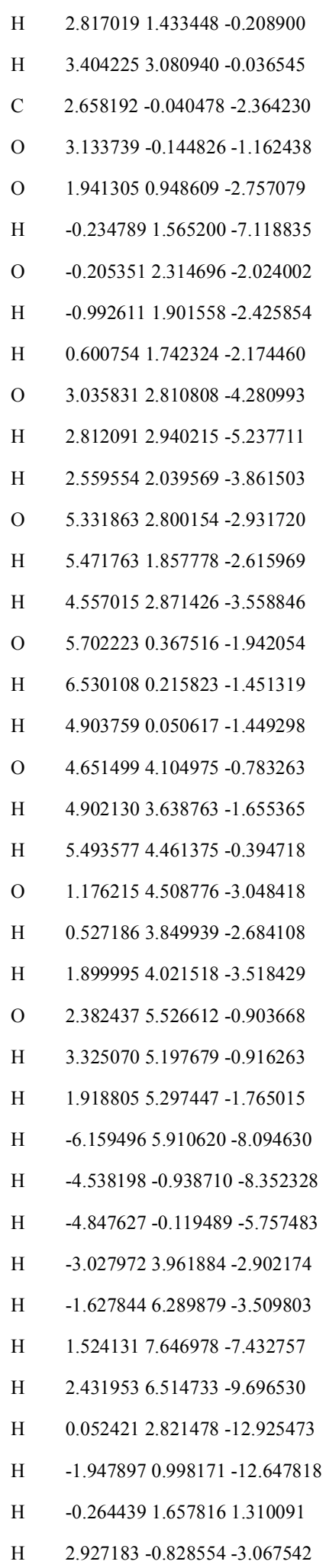

\section{Snapshot 287 ps}

$$
\begin{aligned}
& { }^{2} \mathbf{C}_{\mathrm{R}} \\
& \text { N } \quad-4.4095446 .158653-9.464107 \\
& \text { H } \quad-4.6869136 .882660-10.128877 \\
& \text { C } \quad-5.1750475 .590249-8.444547 \\
& \text { C } \quad-3.1714245 .583396-9.443423 \\
& \text { H } \quad-2.3721865 .847541-10.112511 \\
& \mathrm{~N} \quad-3.1125224 .672489-8.470007 \\
& \text { C } \quad-4.3547394 .666906-7.839856 \\
& \text { H } \quad-4.5447174 .024358-6.998876 \\
& \text { O } \quad-0.1883332 .415431-7.377704 \\
& \text { O } \quad 0.9777463 .039698-6.765028 \\
& \text { H } \quad 0.9412173 .936609-7.156557 \\
& \text { FE } \quad-1.5118083 .542905-7.913849 \\
& \text { N } \quad-2.7629531 .990412-7.606574 \\
& \text { N } \quad-1.7830964 .198412-6.017795 \\
& \text { N } \quad-0.2248295 .105550-8.258129 \\
& \text { N } \quad-1.3414112 .988753-9.867097 \\
& \text { C } \quad-3.1735641 .036222-8.524468 \\
& \text { C } \quad-3.9902640 .022260-7.854218 \\
& \text { C } \quad-4.0885290 .387325-6.542578 \\
& \text { C } \quad-3.3086541 .618920-6.389630 \\
& \text { C } \quad-2.4448493 .513816-5.013265 \\
& \text { C } \quad-2.3638004 .268763-3.757093 \\
& \text { C } \quad-1.6840745 .429715-4.020423 \\
& \text { C } \quad-1.3071745 .365745-5.447067 \\
& \text { C } \quad 0.0151446 .177696-7.405988 \\
& \text { C } \quad 1.0147207 .065147-7.992085 \\
& \text { C } \quad 1.4094006 .512030-9.183038 \\
& \text { C } \quad 0.5591525 .330022-9.392598 \\
& \text { C } \quad-0.5302983 .584878-10.816281 \\
& \text { C } \quad-0.7218962 .937153-12.114643 \\
& \text { C } \quad-1.6416581 .941154-11.934460 \\
& \text { C } \quad-2.0160051 .964403-10.518803 \\
& \text { C } \quad-2.8602361 .059630-9.882419 \\
& \text { H } \quad-3.2940930 .278510-10.498496 \\
& \text { C } \quad-3.1452502 .321816-5.197054 \\
& \text { H } \quad-3.6367231 .897192-4.329009 \\
& \text { C } \quad-0.5351276 .305439-6.134967 \\
& \mathrm{H} \quad-0.2721457 .211630-5.602443 \\
& \text { C } \quad 0.3656554 .630036-10.580847 \\
& \text { H } \quad 0.9107604 .972542-11.450646
\end{aligned}
$$




\begin{tabular}{|c|c|}
\hline $\mathrm{N}$ & 0.6820161 .4153761 .018525 \\
\hline $\mathrm{H}$ & $\begin{array}{llll}0.994861 & 0.567375 & 0.534148\end{array}$ \\
\hline $\mathrm{C}$ & 1.5407292 .4833290 .895292 \\
\hline $\mathrm{N}$ & 1.2832703 .7445771 .279767 \\
\hline $\mathrm{H}$ & 0.3644834 .0743471 .580640 \\
\hline $\mathrm{H}$ & 2.0098634 .4270271 .040676 \\
\hline $\mathrm{N}$ & 2.7396572 .2042310 .384122 \\
\hline $\mathrm{H}$ & $2.8781251 .275175-0.019853$ \\
\hline $\mathrm{H}$ & 3.3515363 .0205840 .179393 \\
\hline $\mathrm{C}$ & $2.323363-0.156377-2.001334$ \\
\hline $\mathrm{O}$ & $2.330592-0.366954-0.721923$ \\
\hline $\mathrm{O}$ & $1.8738980 .945069-2.509650$ \\
\hline $\mathrm{O}$ & $0.3187152 .994983-1.834284$ \\
\hline $\mathrm{H}$ & $0.8259032 .143165-1.998565$ \\
\hline $\mathrm{H}$ & $-0.3778223 .125611-2.507204$ \\
\hline $\mathrm{O}$ & $2.4963212 .589854-4.446153$ \\
\hline $\mathrm{H}$ & $2.1806791 .850029-3.843064$ \\
\hline $\mathrm{H}$ & $1.9264582 .713152-5.241534$ \\
\hline $\mathrm{O}$ & $6.4414434 .590975-2.484968$ \\
\hline $\mathrm{H}$ & $6.2668114 .502674-1.527238$ \\
\hline $\mathrm{H}$ & $5.8502334 .002237-3.023715$ \\
\hline $\mathrm{O}$ & $2.3902024 .594524-2.369759$ \\
\hline $\mathrm{H}$ & $2.7426854 .004595-3.076660$ \\
\hline $\mathrm{H}$ & $1.5401964 .154164-2.060596$ \\
\hline $\mathrm{O}$ & $\begin{array}{llll}5.023483 & 2.739321 & -3.830538\end{array}$ \\
\hline $\mathrm{H}$ & $5.2752291 .858316-3.505270$ \\
\hline $\mathrm{H}$ & $4.0920812 .720477-4.209458$ \\
\hline $\mathrm{O}$ & $3.6630084 .656568-0.043195$ \\
\hline $\mathrm{H}$ & $3.3432764 .792834-0.991129$ \\
\hline $\mathrm{H}$ & 4.5990234 .9209700 .138116 \\
\hline $\mathrm{O}$ & 6.3975775 .1101600 .339359 \\
\hline $\mathrm{H}$ & 6.7276945 .9756080 .032555 \\
\hline $\mathrm{H}$ & 7.0949804 .7089970 .919973 \\
\hline $\mathrm{H}$ & $-6.1676005 .917840-8.135372$ \\
\hline $\mathrm{H}$ & $-4.431428-0.835881-8.361204$ \\
\hline $\mathrm{H}$ & $-4.623937-0.093596-5.723970$ \\
\hline $\mathrm{H}$ & $-2.7538453 .883296-2.815103$ \\
\hline $\mathrm{H}$ & $-1.4175306 .279090-3.391469$ \\
\hline $\mathrm{H}$ & $1.3953747 .943946-7.471622$ \\
\hline $\mathrm{H}$ & $2.2404766 .873186-9.788798$ \\
\hline $\mathrm{H}$ & $-0.1530733 .206177-13.004661$ \\
\hline
\end{tabular}

$$
\begin{array}{ll}
\mathrm{H} & -1.9997851 .223139-12.672203 \\
\mathrm{H} & -0.2824581 .4316381 .282145 \\
\mathrm{H} & 2.722935-0.924912-2.662972
\end{array}
$$$$
{ }^{4} \mathbf{C}_{\mathrm{R}}
$$$$
\text { N } \quad-4.5475466 .209968-9.519365
$$$$
\mathrm{H} \quad-4.8301136 .943440-10.172440
$$$$
\text { C } \quad-5.2883115 .625529-8.492010
$$$$
\text { C } \quad-3.3034735 .643635-9.521240
$$$$
\text { H } \quad-2.5179975 .923509-10.201319
$$$$
\mathrm{N} \quad-3.2144774 .730186-8.556061
$$$$
\text { C } \quad-4.4459724 .708909-7.908195
$$$$
\mathrm{H} \quad-4.6119774 .061130-7.065011
$$$$
\text { O } \quad 0.0359582 .351192-7.211828
$$$$
\text { O } \quad 1.2269973 .029458-6.730969
$$$$
\text { H } \quad 1.1421263 .909939-7.146941
$$$$
\text { FE } \quad-1.4422953 .488252-7.914247
$$$$
\text { N } \quad-2.7164971 .954179-7.596199
$$$$
\mathrm{N} \quad-1.7769704 .176532-6.012150
$$$$
\text { N } \quad-0.1804855 .072803-8.262784
$$$$
\mathrm{N} \quad-1.2869332 .952389-9.866903
$$$$
\text { C } \quad-3.1339781 .005041-8.519802
$$$$
\text { C } \quad-3.9697300 .008505-7.854346
$$$$
\text { C } \quad-4.0754360 .378161-6.542533
$$$$
\text { C } \quad-3.2875841 .596975-6.381755
$$$$
\text { C } \quad-2.4423493 .498733-5.006529
$$$$
\text { C } \quad-2.3629384 .260387-3.754047
$$$$
\text { C } \quad-1.6780905 .417259-4.021469
$$$$
\text { C } \quad-1.2940205 .342396-5.447514
$$$$
\text { C } \quad 0.0459886 .143173-7.404876
$$$$
\text { C } \quad 1.0299707 .044122-7.992989
$$$$
\text { C } \quad 1.4275626 .501019-9.188856
$$$$
\text { C } \quad 0.5938475 .311063-9.400785
$$$$
\text { C } \quad-0.4919903 .563217-10.822552
$$$$
\text { C } \quad-0.7021722 .925332-12.121168
$$$$
\text { C } \quad-1.6180651 .926050-11.936362
$$$$
\text { C } \quad-1.9731801 .933517-10.515900
$$$$
\text { C } \quad-2.8174061 .029270-9.878024
$$$$
\mathrm{H} \quad-3.2618470 .256136-10.496171
$$$$
\text { C } \quad-3.1397852 .306133-5.190344
$$$$
\text { H } \quad-3.6399761 .885587-4.325463
$$

C $\quad-0.5116456 .272437-6.136230$

H $\quad-0.2493107 .179429-5.605377$

C $\quad 0.3988224 .612719-10.590980$

H $\quad 0.9333814 .963791-11.463721$

$\mathrm{N} \quad 0.6857051 .4129421 .019492$

H $\quad 0.9991040 .5640580 .536465$

C $\quad 1.5437942 .4810840 .893906$

$\mathrm{N} \quad 1.2859693 .7431531 .276166$

H $\quad 0.3672994 .0728881 .576929$

H 2.0124264 .4252971 .035116

$\mathrm{N} \quad 2.7424332 .2018460 .382339$

H $\quad 2.8819951 .271406-0.017867$

H $\quad 3.3540033 .0184430 .175937$

C $\quad 2.326319-0.169792-1.990998$

O $\quad 2.333244-0.377665-0.710772$

O $\quad 1.8714140 .928261-2.500079$

O $\quad 0.3122192 .979833-1.823300$

H $\quad 0.8273532 .132709-1.987187$

$\mathrm{H} \quad-0.3717653 .109184-2.508961$

O $\quad 2.5408342 .615087-4.374571$

$\mathrm{H} \quad 2.2117521 .856919-3.803241$

H $\quad 2.0100102 .747618-5.200616$

O $\quad 6.4360514 .596035-2.487018$

H $\quad 6.2615514 .484929-1.531805$

H $\quad 5.8653303 .991863-3.033638$

O $\quad 2.3833664 .593182-2.364696$

H $\quad 2.7144753 .993031-3.078089$

H $\quad 1.5355954 .161723-2.042718$

O $\quad 5.0747672 .720055-3.830607$

H $\quad 5.3370151 .839773-3.512422$

H $\quad 4.1255312 .707502-4.167867$

O $\quad 3.6645254 .653096-0.047891$

H $\quad 3.3444914 .787645-0.996652$

H $\quad 4.6001494 .9189920 .133738$

O $\quad 6.3972035 .1038120 .333781$

H $\quad 6.7377355 .950546-0.011319$

H $\quad 7.0936634 .7132640 .922470$

H $\quad-6.2695455 .951687-8.147240$

H $\quad-4.418310-0.846143-8.360724$

H $\quad-4.621418-0.096797-5.727435$

H $\quad-2.7542163 .879774-2.810595$ 


\begin{tabular}{|c|c|}
\hline $\mathrm{H}$ & $-1.4109406 .268145-3.394819$ \\
\hline $\mathrm{H}$ & $1.3993857 .928821-7.474427$ \\
\hline $\mathrm{H}$ & $2.2489406 .877592-9.798443$ \\
\hline $\mathrm{H}$ & $-0.1462103 .204622-13.016137$ \\
\hline $\mathrm{H}$ & $-1.9836721 .213259-12.675501$ \\
\hline $\mathrm{H}$ & -0.2789071 .4299531 .282563 \\
\hline $\mathrm{H}$ & $2.730262-0.937898-2.650475$ \\
\hline \multicolumn{2}{|c|}{${ }^{6} \mathbf{C}_{\mathrm{R}}$} \\
\hline $\mathrm{N}$ & $-4.5112186 .192196-9.499538$ \\
\hline $\mathrm{H}$ & $-4.7945826 .923640-10.154067$ \\
\hline $\mathrm{C}$ & $-5.2549695 .613078-8.469159$ \\
\hline $\mathrm{C}$ & $-3.2691925 .622566-9.499972$ \\
\hline 11 & $-2.4824035 .897520-10.180348$ \\
\hline 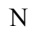 & $-3.1829764 .710730-8.531273$ \\
\hline $\mathrm{C}$ & $-4.4152804 .695682-7.882390$ \\
\hline $\mathrm{H}$ & $-4.5845034 .051716-7.037261$ \\
\hline $\mathrm{O}$ & $0.0413092 .382802-7.177520$ \\
\hline $\mathrm{O}$ & $1.2348463 .061019-6.743813$ \\
\hline $\mathrm{H}$ & $1.1323283 .941672-7.164080$ \\
\hline FE & $-1.4202123 .478680-7.903156$ \\
\hline $\mathbb{N}$ & $-2.7298241 .920737-7.592886$ \\
\hline $\mathrm{N}$ & $-1.7890484 .211419-5.965290$ \\
\hline $\mathrm{N}$ & -0.1346615 .10775 \\
\hline $\mathrm{N}$ & $-1.2551922 .933574-9.919425$ \\
\hline $\mathrm{C}$ & $-3.1263340 .974788-8.523168$ \\
\hline $\mathrm{C}$ & $-3.966507-0.019572-7.851647$ \\
\hline$c$ & $-4.0837140 .357423-6.540847$ \\
\hline $\mathrm{C}$ & $-3.3023231 .585359-6.375676$ \\
\hline $\mathrm{C}$ & $-2.4671353 .527063-4.978910$ \\
\hline $\mathrm{C}$ & $-2.3794844 .288415-3.720300$ \\
\hline $\mathrm{C}$ & $-1.6721905 .434805-3.981477$ \\
\hline $\mathrm{C}$ & $-1.2815975 .363903-5.410587$ \\
\hline $\mathrm{C}$ & $0.0857056 .166514-7.396480$ \\
\hline $\mathrm{C}$ & $1.0584137 .077114-7.997528$ \\
\hline $\mathrm{C}$ & $1.4373556 .549020-9.208310$ \\
\hline $\mathrm{C}$ & $0.6099685 .350284-9.422770$ \\
\hline $\mathrm{C}$ & $-0.4691003 .561167-10.863880$ \\
\hline $\mathrm{C}$ & $-0.6748452 .912097-12.163381$ \\
\hline $\mathrm{C}$ & $-1.5781881 .900379-11.973592$ \\
\hline $\mathrm{C}$ & $.9358421 .904772-10.54811$ \\
\hline
\end{tabular}

\begin{tabular}{|c|c|c|c|}
\hline $\mathrm{C}$ & $-2.7799581 .003098-9.884669$ & $\mathrm{H}$ & $-6.2392985 .938770-8.132873$ \\
\hline $\mathrm{H}$ & $-3.2130390 .219769-10.499639$ & $\mathrm{H}$ & $-4.418451-0.871906-8.358934$ \\
\hline $\mathrm{C}$ & $-3.1611212 .324513-5.188959$ & $\mathrm{H}$ & $-4.634367-0.115610-5.727774$ \\
\hline $\mathrm{H}$ & $-3.6702551 .906462-4.327015$ & $\mathrm{H}$ & $-2.7798843 .908422-2.780433$ \\
\hline $\mathrm{C}$ & $-0.4745416 .276140-6.115847$ & $\mathrm{H}$ & $-1.3933966 .283695-3.357197$ \\
\hline $\mathrm{H}$ & $-0.1994157 .179055-5.582032$ & $\mathrm{H}$ & $1.4289967 .960165-7.476996$ \\
\hline $\mathrm{C}$ & $0.4016144 .636198-10.613016$ & $\mathrm{H}$ & $2.2494326 .933801-9.825191$ \\
\hline $\mathrm{H}$ & $0.9251804 .998622-11.488903$ & $\mathrm{H}$ & $-0.1261493 .198571-13.060561$ \\
\hline $\mathrm{N}$ & 0.6871891 .4096871 .022377 & $\mathrm{H}$ & $-1.9491521 .190465-12.712831$ \\
\hline $\mathrm{H}$ & 1.0001610 .5599580 .541156 & $\mathrm{H}$ & -0.2778671 .4273631 .283770 \\
\hline $\mathrm{C}$ & 1.5455132 .4774430 .895380 & $\mathrm{H}$ & $2.729054-0.945039-2.651742$ \\
\hline $\mathrm{N}$ & 1.2882813 .7396901 .277115 & \multirow{2}{*}{\multicolumn{2}{|c|}{${ }^{2} \mathbf{C}_{\mathrm{Ia}}$}} \\
\hline $\mathrm{H}$ & 0.3698634 .0699651 .577895 & & \\
\hline $\mathrm{H}$ & 2.0151664 .4214561 .036083 & $\mathrm{~N}$ & $-4.4611766 .172585-9.481363$ \\
\hline $\mathrm{N}$ & 2.7440852 .1972580 .383911 & $\mathrm{H}$ & $-4.7376226 .901565-10.141667$ \\
\hline $\mathrm{H}$ & $2.8833191 .267236-0.016820$ & $\mathrm{C}$ & $-5.2192425 .603655-8.456240$ \\
\hline $\mathrm{H}$ & 3.3566563 .0134630 .178306 & $\mathrm{C}$ & $-3.2252905 .592934-9.471590$ \\
\hline $\mathrm{C}$ & $2.323942-0.177355-1.992490$ & $\mathrm{H}$ & $-2.4308095 .857422-10.146486$ \\
\hline $\mathrm{O}$ & $2.333844-0.384233-0.712709$ & $\mathrm{~N}$ & $-3.1584604 .681465-8.499493$ \\
\hline $\mathrm{O}$ & $1.8635850 .918969-2.502262$ & $\mathrm{C}$ & $-4.3954334 .679583-7.859211$ \\
\hline $\mathrm{O}$ & $0.3022482 .971476-1.818087$ & $\mathrm{H}$ & $-4.5798374 .036990-7.017083$ \\
\hline $\mathrm{H}$ & $0.8104192 .121919-1.987325$ & $\mathrm{O}$ & $-0.3097812 .491034-7.479531$ \\
\hline $\mathrm{H}$ & $-0.3949273 .103677-2.489821$ & $\mathrm{O}$ & $1.8591083 .528868-6.598160$ \\
\hline $\mathrm{O}$ & $2.5722642 .597480-4.360682$ & $\mathrm{H}$ & $1.1763664 .056427-7.066309$ \\
\hline $\mathrm{H}$ & $2.2272701 .843638-3.789322$ & $\mathrm{FE}$ & $-1.5101053 .485159-7.936386$ \\
\hline $\mathrm{H}$ & $2.0454912 .747236-5.182020$ & $\mathrm{~N}$ & $-2.8392091 .996191-7.629945$ \\
\hline $\mathrm{O}$ & $6.4486224 .576449-2.481869$ & $\mathrm{~N}$ & $-1.8264174 .158456-6.034215$ \\
\hline $\mathrm{H}$ & $6.2755074 .460428-1.527006$ & $\mathrm{~N}$ & $-0.2770935 .080112-8.293611$ \\
\hline $\mathrm{H}$ & $5.8895123 .962328-3.029358$ & $\mathrm{~N}$ & $-1.3713742 .974921-9.895601$ \\
\hline $\mathrm{O}$ & $2.3817404 .575387-2.359984$ & $\mathrm{C}$ & $-3.2288631 .036083-8.550943$ \\
\hline $\mathrm{H}$ & $2.7165853 .972366-3.069232$ & $\mathrm{C}$ & $-4.0299930 .011762-7.880448$ \\
\hline $\mathrm{H}$ & $1.5310744 .148157-2.040145$ & $\mathrm{C}$ & $-4.1305590 .369963-6.566976$ \\
\hline $\mathrm{O}$ & $5.1136042 .687798-3.828008$ & $\mathrm{C}$ & $-3.3685121 .608694-6.409169$ \\
\hline $\mathrm{H}$ & $5.3944101 .802501-3.541740$ & $\mathrm{C}$ & $-2.4870183 .480186-5.025922$ \\
\hline $\mathrm{H}$ & $4.1611792 .676262-4.152201$ & $\mathrm{C}$ & $-2.3948934 .233928-3.770300$ \\
\hline $\mathrm{O}$ & $3.6670534 .647289-0.046476$ & $\mathrm{C}$ & $-1.7094885 .389951-4.037082$ \\
\hline $\mathrm{H}$ & $3.3436174 .778808-0.994458$ & $\mathrm{C}$ & $-1.3424875 .324841-5.466951$ \\
\hline $\mathrm{H}$ & 4.6027404 .9154330 .131336 & $\mathrm{C}$ & $-0.0357896 .139414-7.430912$ \\
\hline $\mathrm{O}$ & 6.4023015 .0984500 .327435 & $\mathrm{C}$ & $0.9767097 .023176-8.002669$ \\
\hline $\mathrm{H}$ & $6.7452395 .936527-0.036035$ & $\mathrm{C}$ & $1.3838156 .470546-9.189396$ \\
\hline $\mathrm{H}$ & 7.0983124 .7169600 .922720 & $\mathrm{C}$ & $0.5295405 .295843-9.412287$ \\
\hline
\end{tabular}




\begin{tabular}{|c|c|}
\hline$C$ & $-0.5455803 .556670-10.839308$ \\
\hline & $-0.7312882 .901376-12.132765$ \\
\hline & $-1.6597291 .914070-11.952020$ \\
\hline & $-2.0471161 .949123-10.540445$ \\
\hline & $-2.9016891 .053751-9.905133$ \\
\hline & $-3.3285890 .268285-10.520014$ \\
\hline & $-3.1962432 .295737-5.210488$ \\
\hline & $-3.6782721 .862900-4.341628$ \\
\hline & $-0.5798846 .264816-6.157627$ \\
\hline & $-0.3000657 .161010-5.619079$ \\
\hline & $0.3514594 .597369-10.601630$ \\
\hline & $0.9105774 .932382-11.465021$ \\
\hline & 0.6899661 .4141381 .015885 \\
\hline & 1.0033470 .5719570 .517900 \\
\hline & 1.5509632 .4814020 .911106 \\
\hline & 1.2956843 .7390041 .308056 \\
\hline & 0.3732584 .0697361 .595879 \\
\hline 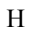 & 2.0325494 .4196351 .088901 \\
\hline & 2.7539802 .2059270 .404907 \\
\hline 1 & $2.8977851 .280820-0.001928$ \\
\hline & 3.3686543 .0236400 .218153 \\
\hline & $2.287073-0.148505-1.983540$ \\
\hline & $2.297053-0.372759-0.706286$ \\
\hline h & $1.8174030 .949170-2.480161$ \\
\hline D & $0.2863203 .024325-1.794508$ \\
\hline H & $0.7883392 .170765-1.960482$ \\
\hline & $-0.3840223 .173085-2.489954$ \\
\hline 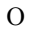 & $2.7395132 .693562-4.160773$ \\
\hline & $2.3077601 .923256-3.678596$ \\
\hline & $2.3018472 .957030-5.005873$ \\
\hline & $6.5497684 .631740-2.402701$ \\
\hline $\mathrm{H}$ & $6.3746024 .622072-1.440601$ \\
\hline $\mathrm{H}$ & $6.0246823 .928772-2.872501$ \\
\hline & $2.3889464 .644477-2.254475$ \\
\hline & $2.7518864 .017621-2.935532$ \\
\hline & $1.5302004 .221451-1.955984$ \\
\hline & $5.2629212 .620806-3.588969$ \\
\hline$\pi$ & $5.5642901 .711197-3.433798$ \\
\hline & $4.3095952 .652379-3.908292$ \\
\hline & 3.6614114 .6788370 .051751 \\
\hline & $3.3309564 .826668-0.893940$ \\
\hline
\end{tabular}

$$
\begin{array}{ll}
\mathrm{H} & 4.6018284 .9313300 .225308 \\
\mathrm{O} & 6.3948615 .1569170 .438931 \\
\mathrm{H} & 6.6832616 .0684840 .242744 \\
\mathrm{H} & 7.1099854 .7233720 .973843 \\
\mathrm{H} & -6.2075705 .932033-8.134613 \\
\mathrm{H} & -4.464590-0.849101-8.388491 \\
\mathrm{H} & -4.657707-0.120012-5.748377 \\
\mathrm{H} & -2.7833033 .850504-2.826801 \\
\mathrm{H} & -1.4286766 .234349-3.407636 \\
\mathrm{H} & 1.3563297 .898902-7.476304 \\
\mathrm{H} & 2.2242846 .829320-9.783499 \\
\mathrm{H} & -0.1546303 .163280-13.019860 \\
\mathrm{H} & -2.0200701 .197043-12.689647 \\
\mathrm{H} & -0.2750401 .4316031 .277475 \\
\mathrm{H} & 2.697753-0.904094-2.653236
\end{array}
$$$$
{ }^{2} \mathbf{C}_{\mathrm{Ib}}
$$$$
\text { N } \quad-4.4870186 .168036-9.491979
$$$$
\mathrm{H} \quad-4.7625426 .902649-10.146515
$$$$
\text { C } \quad-5.2431485 .592199-8.469323
$$$$
\text { C } \quad-3.2498385 .592342-9.486197
$$$$
\text { H } \quad-2.4567205 .862818-10.160062
$$$$
\text { N } \quad-3.1803294 .675339-8.519611
$$$$
\text { C } \quad-4.4169444 .665785-7.878675
$$$$
\text { H } \quad-4.5995144 .018908-7.039327
$$$$
\text { O } \quad-0.1874512 .687812-7.442706
$$$$
\text { O } \quad 1.4086913 .776656-5.731680
$$$$
\text { H } \quad 0.7828593 .355108-6.408285
$$$$
\text { FE } \quad-1.5037053 .518185-7.959081
$$$$
\text { N } \quad-2.7943621 .989602-7.641282
$$$$
\text { N } \quad-1.8469004 .186033-6.061549
$$$$
\text { N } \quad-0.3390765 .143803-8.356861
$$$$
\text { N } \quad-1.3723082 .987879-9.917254
$$$$
\text { C } \quad-3.1840521 .022518-8.556593
$$$$
\text { C } \quad-4.0012000 .008899-7.887592
$$$$
\text { C } \quad-4.1176660 .381167-6.579731
$$$$
\text { C } \quad-3.3525611 .619392-6.424278
$$$$
\text { C } \quad-2.5060183 .512091-5.052612
$$$$
\text { C } \quad-2.3910604 .255727-3.792785
$$$$
\text { C } \quad-1.6767575 .395067-4.055713
$$$$
\text { C } \quad-1.3356305 .342687-5.492904
$$

C $\quad-0.0494776 .156895-7.467046$

C $\quad 0.9929137 .024111-8.020810$

C $\quad 1.3766286 .487130-9.220095$

C $\quad 0.4808335 .342725-9.461165$

C $\quad-0.5793633 .592644-10.877807$

C $\quad-0.7658072 .929762-12.166822$

C $\quad-1.6649211 .918632-11.971605$

C $\quad-2.0317391 .944567-10.554173$

C $\quad-2.8620191 .033712-9.911586$

H $\quad-3.2856470 .242797-10.521528$

C $\quad-3.2097822 .321936-5.233823$

H $\quad-3.7043791 .896186-4.369154$

C $\quad-0.5848606 .282355-6.191000$

H $\quad-0.2688947 .159179-5.642357$

C $\quad 0.3004384 .648711-10.653181$

H $\quad 0.8500494 .989848-11.520188$

$\mathrm{N} \quad 0.6704961 .3913410 .960758$

H $\quad 0.9790570 .5502580 .450080$

C $\quad 1.5456872 .4476730 .883434$

$\mathrm{N} \quad 1.3019543 .7048571 .290524$

H $\quad 0.3820604 .0439391 .574509$

$\mathrm{H} \quad 2.0521684 .3776401 .088697$

$\mathrm{N} \quad 2.7530142 .1635280 .390551$

H $\quad 2.8996541 .241048-0.014541$

$\mathrm{H} \quad 3.3838342 .9721130 .225347$

C $\quad 2.234519-0.204841-1.974529$

O $\quad 2.191786-0.449952-0.701901$

O $\quad 1.8111230 .911431-2.470928$

O $\quad 0.3807423 .079626-1.926383$

$\mathrm{H} \quad 0.8107632 .182201-2.056634$

$\mathrm{H} \quad-0.2717753 .270102-2.627910$

O $\quad 3.1021552 .494052-4.088148$

$\mathrm{H} \quad 2.5679501 .809707-3.576862$

H $\quad 2.5779852 .899237-4.828262$

O $\quad 6.5979634 .529826-2.274651$

H $\quad 6.4525474 .614630-1.310931$

H $\quad 6.1557673 .711766-2.632031$

O $\quad 2.5924534 .548859-2.279012$

H $\quad 3.0330673 .892465-2.878720$

H $\quad 1.7006024 .145749-2.048108$

O $\quad 5.5080652 .303181-3.229837$ 


\begin{tabular}{ll}
$\mathrm{H}$ & $6.0449241 .556455-3.539227$ \\
$\mathrm{H}$ & $4.6164772 .367978-3.691989$ \\
$\mathrm{O}$ & 3.6980264 .6393700 .107498 \\
$\mathrm{H}$ & $3.4190664 .776872-0.856776$ \\
$\mathrm{H}$ & 4.6372894 .8732510 .311139 \\
$\mathrm{O}$ & 6.4119565 .1498270 .554459 \\
$\mathrm{H}$ & 6.6578786 .0863710 .432261 \\
$\mathrm{H}$ & 7.1455614 .7058321 .055962 \\
$\mathrm{H}$ & $-6.2274415 .924888-8.139853$ \\
$\mathrm{H}$ & $-4.441190-0.851260-8.392172$ \\
$\mathrm{H}$ & $-4.658344-0.098485-5.763847$ \\
$\mathrm{H}$ & $-2.7796573 .878035-2.847054$ \\
$\mathrm{H}$ & $-1.3485626 .215538-3.417604$ \\
$\mathrm{H}$ & $1.3992997 .870341-7.466905$ \\
$\mathrm{H}$ & $2.2263156 .830557-9.810145$ \\
$\mathrm{H}$ & $-0.2040623 .201225-13.060588$ \\
$\mathrm{H}$ & $-2.0224681 .196604-12.705704$ \\
$\mathrm{H}$ & -0.2882371 .4110521 .244334 \\
$\mathrm{H}$ & $2.651974-0.952376-2.649059$ \\
\hline &
\end{tabular}

\section{${ }^{2} \mathrm{C}_{\mathrm{R}} \mathrm{H}$}

\begin{tabular}{|c|c|c|c|}
\hline $\mathrm{C}$ & $-2.4543333 .549369-4.980315$ & $\mathrm{H}$ & $6.0039963 .655239-2.507837$ \\
\hline $\mathrm{C}$ & $-2.3701714 .314156-3.744043$ & $\mathrm{O}$ & $2.6393204 .836147-2.504731$ \\
\hline $\mathrm{C}$ & $-1.6555985 .456527-4.009708$ & $\mathrm{H}$ & $3.1254974 .221475-3.102254$ \\
\hline $\mathrm{C}$ & $-1.2642795 .382784-5.421134$ & $\mathrm{H}$ & $1.7708714 .376081-2.279585$ \\
\hline $\mathrm{C}$ & $0.0349086 .161472-7.397543$ & $\mathrm{O}$ & $5.3670762 .168733-3.054347$ \\
\hline $\mathrm{C}$ & $1.0514327 .033929-7.974707$ & $\mathrm{H}$ & $6.0148131 .518018-3.373022$ \\
\hline $\mathrm{C}$ & $1.4406786 .481709-9.166383$ & $\mathrm{H}$ & $4.6284972 .332616-3.702568$ \\
\hline $\mathrm{C}$ & $0.5644595 .326003-9.385130$ & $\mathrm{O}$ & $3.7028834 .690798-0.035670$ \\
\hline $\mathrm{C}$ & $-0.5029683 .587076-10.802776$ & $\mathrm{H}$ & $3.4634174 .906136-0.988402$ \\
\hline $\mathrm{C}$ & $-0.7105652 .939235-12.090596$ & $\mathrm{H}$ & 4.6426134 .8888100 .205088 \\
\hline $\mathrm{C}$ & $-1.6260821 .941111-11.898337$ & $\mathrm{O}$ & 6.4245605 .1696590 .502161 \\
\hline $\mathrm{C}$ & $-1.9869621 .965083-10.483215$ & $\mathrm{H}$ & 6.6289606 .1243980 .516049 \\
\hline $\mathrm{C}$ & $-2.8440691 .077508-9.847522$ & $\mathrm{H}$ & 7.1515654 .7022230 .994290 \\
\hline $\mathrm{H}$ & $-3.2927000 .303967-10.460751$ & $\mathrm{H}$ & $-6.1593645 .906686-8.149879$ \\
\hline $\mathrm{C}$ & $-3.1947622 .387185-5.169705$ & $\mathrm{H}$ & $-4.517146-0.723940-8.349488$ \\
\hline $\mathrm{H}$ & $-3.7347431 .996024-4.315860$ & $\mathrm{H}$ & $-4.7815560 .067964-5.740557$ \\
\hline $\mathrm{C}$ & $-0.4794716 .298337-6.114547$ & $\mathrm{H}$ & $-2.7650523 .936828-2.800772$ \\
\hline $\mathrm{H}$ & $-0.1510387 .172848-5.570289$ & $\mathrm{H}$ & $-1.3677316 .296533-3.377578$ \\
\hline $\mathrm{C}$ & $0.3829024 .633477-10.576673$ & $\mathrm{H}$ & $1.4350927 .913148-7.457170$ \\
\hline $\mathrm{H}$ & $0.9292964 .977376-11.443827$ & $\mathrm{H}$ & $2.2779596 .833506-9.769099$ \\
\hline $\mathrm{N}$ & 0.6256791 .4414070 .884047 & $\mathrm{H}$ & $-0.1667863 .220831-12.992303$ \\
\hline $\mathrm{H}$ & 0.9213200 .6107250 .358650 & $\mathrm{H}$ & $-1.9942721 .227861-12.635748$ \\
\hline $\mathrm{C}$ & 1.4961152 .5040270 .805290 & $\mathrm{H}$ & -0.3094681 .4277701 .238006 \\
\hline $\mathrm{N}$ & 1.2543853 .7487391 .248259 & $\mathrm{H}$ & $2.587767-0.781749-2.861940$ \\
\hline
\end{tabular}

H $\quad 0.3462824 .0748321 .586132$

H $\quad 1.9890574 .4328181 .049080$

$\mathrm{N} \quad 2.6858112 .2466420 .260888$

H $\quad 2.8377171 .334518-0.162837$

H $\quad 3.3086133 .0631270 .086991$

C $\quad 2.187739-0.036278-2.174687$

O $\quad 2.141318-0.311284-0.918536$

O $\quad 1.8005021 .099324-2.668183$

H $\quad 0.1271291 .543524-7.074893$

O $\quad 0.5155033 .309481-2.086841$

H $\quad 0.9029482 .387919-2.198226$

$\mathrm{H} \quad-0.2895753 .439674-2.619661$

O $\quad 3.1182172 .624539-4.306608$

H $\quad 2.5547571 .985154-3.758292$

H $\quad 2.6475052 .909074-5.112126$

O $\quad 6.4269564 .528754-2.300789$

H $\quad 6.4144084 .704286-1.335507$

\footnotetext{
$6.0039963 .655239-2.507837$

$2.6393204 .836147-2.50473$

$3.1254974 .221475-3.102254$

$1.7708714 .376081-2.279585$

$5.3670762 .168733-3.054347$

$6.0148131 .518018-3.373022$

$4.6284972 .332616-3.702568$

$3.7028834 .690798-0.035670$

$3.4634174 .906136-0.988402$

4.6426134 .8888100 .205088

6.4245605 .1696590 .502161

6.6289606 .1243980 .516049

7.1515654 .7022230 .994290

$-6.1593645 .906686-8.149879$

$-4.517146-0.723940-8.349488$

$-4.7815560 .067964-5.740557$

$-2.7650523 .936828-2.800772$

$-1.3677316 .296533-3.377578$

$1.4350927 .913148-7.457170$

$2.2779596 .833506-9.769099$

$-0.1667863 .220831-12.992303$

$-0.3094681 .4277701 .238006$

H $\quad 2.587767-0.781749-2.861940$
}

\section{${ }^{4} \mathbf{C}_{\mathrm{R}} \mathbf{H}$}

$\mathrm{N} \quad-4.5781416 .223650-9.512498$

H $\quad-4.8608156 .968816-10.156442$

C $\quad-5.3220365 .613675-8.501853$

C $\quad-3.3321615 .679747-9.524160$

H $\quad-2.5471425 .991075-10.191836$

N $\quad-3.2457884 .742072-8.576049$

C $\quad-4.4801334 .689216-7.931591$

H $\quad-4.6522824 .024611-7.102564$

O $\quad 0.6115212 .474110-7.064057$

O $\quad 1.0950593 .000871-5.804114$

$\mathrm{H} \quad 0.4674783 .739659-5.669665$

FE $\quad-1.5564913 .613611-7.995307$

N $\quad-2.6635381 .980719-7.608161$

$\mathrm{N} \quad-1.7068514 .169885-6.041171$

$\mathrm{N} \quad-0.2086605 .086599-8.304353$ 


\begin{tabular}{|c|c|}
\hline I & $-1.2393492 .936045-9.857888$ \\
\hline $\mathrm{C}$ & $-3.0971181 .016540-8.527047$ \\
\hline $\mathrm{C}$ & $-3.9875700 .070769-7.872290$ \\
\hline $\mathrm{C}$ & $-4.1307220 .474453-6.573323$ \\
\hline 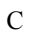 & $-3.3014671 .658573-6.404397$ \\
\hline 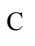 & $-2.4214143 .525157-5.031901$ \\
\hline $\mathrm{C}$ & $-2.3298854 .279442-3.788914$ \\
\hline $\mathrm{C}$ & $-1.6045805 .416923-4.045665$ \\
\hline $\mathrm{C}$ & $-1.2130585 .345312-5.457226$ \\
\hline $\mathrm{C}$ & $0.0839386 .128873-7.429100$ \\
\hline $\mathrm{C}$ & $1.0910537 .002041-8.010670$ \\
\hline C & $1.4741166 .453553-9.207387$ \\
\hline $\mathrm{C}$ & $0.6008875 .297841-9.425572$ \\
\hline $\mathrm{C}$ & $-0.4591923 .551570-10.836613$ \\
\hline $\mathrm{C}$ & $-0.6672732 .903151-12.120578$ \\
\hline $\mathrm{C}$ & $-1.5662181 .889343-11.924798$ \\
\hline $\mathrm{C}$ & $-1.9143451 .898085-10.510790$ \\
\hline $\mathrm{C}$ & $-2.7589811 .000442-9.874986$ \\
\hline $\mathrm{H}$ & $-3.1992580 .220993-10.485667$ \\
\hline $\mathrm{C}$ & $-3.1627882 .363684-5.216605$ \\
\hline $\mathrm{H}$ & $-3.7065701 .976547-4.363126$ \\
\hline $\mathrm{C}$ & $-0.4211916 .261400-6.143290$ \\
\hline $\mathrm{H}$ & $-0.0824647 .127897-5.592714$ \\
\hline $\mathrm{C}$ & $0.4173744 .606249-10.616682$ \\
\hline $\mathrm{H}$ & $0.9555374 .954972-11.486553$ \\
\hline $\mathrm{N}$ & 0.6347021 .4238250 .915446 \\
\hline $\mathrm{H}$ & 0.9362610 .5856890 .405831 \\
\hline $\mathrm{C}$ & 1.5035332 .4870700 .830290 \\
\hline I & 1.2563013 .7365951 .256468 \\
\hline $\mathrm{H}$ & 0.3470304 .0647381 .588959 \\
\hline $\mathrm{H}$ & 1.9930494 .4190351 .058469 \\
\hline $\mathrm{N}$ & 2.6978062 .2254320 .298082 \\
\hline $\mathrm{H}$ & $2.8553951 .308236-0.111852$ \\
\hline $\mathrm{H}$ & 3.3219323 .0402080 .122373 \\
\hline $\mathrm{C}$ & $2.241424-0.097975-2.108760$ \\
\hline U & $2.168623-0.366703-0.853076$ \\
\hline U & $1.8856961 .045140-2.609034$ \\
\hline $\mathrm{H}$ & $0.5063481 .519661-6.834626$ \\
\hline $\mathrm{O}$ & $0.5475563 .238186-2.092895$ \\
\hline $\mathrm{H}$ & $0.9548922 .322818-2.187376$ \\
\hline H & $-0.2326743 .351765-2.665052$ \\
\hline
\end{tabular}

$$
\begin{array}{ll}
\mathrm{O} & 3.2873792 .557394-4.190574 \\
\mathrm{H} & 2.7096501 .925982-3.649312 \\
\mathrm{H} & 2.8077792 .864943-4.983515 \\
\mathrm{O} & 6.4637264 .556312-2.315106 \\
\mathrm{H} & 6.4430064 .746177-1.352798 \\
\mathrm{H} & 6.0961983 .651428-2.500123 \\
\mathrm{O} & 2.6676004 .783882-2.494080 \\
\mathrm{H} & 3.1895334 .156471-3.048954 \\
\mathrm{H} & 1.8023914 .314553-2.277148 \\
\mathrm{O} & 5.5375412 .130813-2.994338 \\
\mathrm{H} & 6.2023581 .501268-3.319581 \\
\mathrm{H} & 4.7825212 .273465-3.630801 \\
\mathrm{O} & 3.7120334 .672772-0.009656 \\
\mathrm{H} & 3.4707344 .882736-0.962282 \\
\mathrm{H} & 4.6536644 .8737210 .224166 \\
\mathrm{O} & 6.4250085 .1788490 .502807 \\
\mathrm{H} & 6.6169706 .1356700 .536890 \\
\mathrm{H} & 7.1568314 .7102830 .986785 \\
\mathrm{H} & -6.3008855 .934588-8.145572 \\
\mathrm{H} & -4.461734-0.771724-8.375718 \\
\mathrm{H} & -4.7334680 .038819-5.776463 \\
\mathrm{H} & -2.7296953 .901503-2.847967 \\
\mathrm{H} & -1.3160486 .253268-3.408999 \\
\mathrm{H} & 1.4693337 .884708-7.495043 \\
\mathrm{H} & 2.3075736 .810208-9.812543 \\
\mathrm{H} & -0.1423523 .200393-13.028416 \\
\mathrm{H} & -1.9438871 .186206-12.667113 \\
\mathrm{H} & -0.3040631 .4143721 .259834 \\
\mathrm{H} & -0.850064-2.791004
\end{array}
$$

\section{${ }^{6} \mathbf{C}_{\mathrm{R}} \mathrm{H}$}

$$
\begin{array}{ll}
\mathrm{N} & -4.5671376 .230601-9.508866 \\
\mathrm{H} & -4.8544586 .973784-10.153937 \\
\mathrm{C} & -5.3075215 .618731-8.495486 \\
\mathrm{C} & -3.3205925 .694773-9.525595 \\
\mathrm{H} & -2.5388346 .010224-10.195291 \\
\mathrm{~N} & -3.2305204 .756707-8.575418 \\
\mathrm{C} & -4.4627904 .697644-7.926555 \\
\mathrm{H} & -4.6273904 .031477-7.097428 \\
\mathrm{O} & 0.5997912 .442730-7.076809 \\
\mathrm{O} & 1.0593392 .941554-5.796528
\end{array}
$$

H $\quad 0.3733983 .620843-5.626507$

FE $\quad-1.5675153 .650775-8.016555$

N $\quad-2.6489741 .942850-7.597788$

N $\quad-1.6562234 .173624-5.989848$

$\mathrm{N} \quad-0.1434685 .116237-8.315421$

N $\quad-1.1915152 .908910-9.898522$

C $\quad-3.0754100 .985663-8.522415$

C $\quad-3.9763060 .047120-7.863434$

C $\quad-4.1208390 .454353-6.563657$

C $\quad-3.2836871 .636973-6.390303$

C $\quad-2.3912943 .530960-4.997238$

C $\quad-2.3051964 .294878-3.756127$

C $\quad-1.5589735 .422331-4.008836$

C $\quad-1.1490925 .344635-5.417533$

C $\quad 0.1614956 .136476-7.420316$

C $\quad 1.1565777 .019061-8.015759$

C $\quad 1.5137536 .488570-9.230755$

C $\quad 0.6422255 .326502-9.449011$

C $\quad-0.4105853 .533091-10.868369$

C $\quad-0.6193792 .874575-12.152486$

C $\quad-1.5165731 .857721-11.953389$

C $\quad-1.8668601 .864705-10.535347$

C $\quad-2.7156410 .974796-9.875265$

H $\quad-3.1526270 .189058-10.482612$

C $\quad-3.1366342 .363648-5.206000$

H $\quad-3.6883441 .981472-4.354580$

C $\quad-0.3355856 .243297-6.119892$

H $\quad 0.0255687 .097587-5.562640$

C $\quad 0.4462564 .612265-10.636346$

H $\quad 0.9739134 .965975-11.511864$

$\mathrm{N} \quad 0.6368191 .4175870 .916460$

H $\quad 0.9388120 .5800110 .405846$

C $\quad 1.5071382 .4798980 .835972$

$\mathrm{N} \quad 1.2601153 .7291641 .263147$

$\mathrm{H} \quad 0.3502474 .0581371 .592705$

H 1.9986494 .4108241 .067554

$\mathrm{N} \quad 2.7028632 .2174360 .307296$

$\mathrm{H} \quad 2.8607051 .301067-0.104471$

$\mathrm{H} \quad 3.3281203 .0318130 .135393$

C $\quad 2.249726-0.103442-2.104386$

O $\quad 2.172327-0.373976-0.848996$ 


\begin{tabular}{|c|c|}
\hline $\mathrm{O}$ & $1.8950021 .039570-2.604655$ \\
\hline $\mathrm{H}$ & $0.4907421 .483478-6.869354$ \\
\hline $\mathrm{O}$ & $0.5692223 .239168-2.082866$ \\
\hline $\mathrm{H}$ & $0.9718432 .321996-2.180815$ \\
\hline $\mathrm{H}$ & $-0.2196393 .355557-2.642264$ \\
\hline $\mathrm{O}$ & $3.2882082 .560191-4.188920$ \\
\hline $\mathrm{H}$ & $2.7141381 .925317-3.648657$ \\
\hline $\mathrm{H}$ & $2.8029022 .869478-4.977853$ \\
\hline $\mathrm{O}$ & $6.4534894 .529262-2.283362$ \\
\hline $\mathrm{H}$ & $6.4352324 .725074-1.322222$ \\
\hline $\mathrm{H}$ & $6.0850903 .623850-2.463112$ \\
\hline $\mathrm{O}$ & $2.6909044 .784657-2.473353$ \\
\hline $\mathrm{H}$ & $3.2115974 .160951-3.033112$ \\
\hline $\mathrm{H}$ & $1.8231604 .316534-2.264247$ \\
\hline $\mathrm{O}$ & $5.5220432 .104441-2.961928$ \\
\hline $\mathrm{H}$ & $6.1903031 .475212-3.281038$ \\
\hline $\mathrm{H}$ & $4.7823592 .259323-3.612955$ \\
\hline $\mathrm{O}$ & 3.7155084 .6676450 .012753 \\
\hline $\mathrm{H}$ & $3.4798374 .876783-0.942302$ \\
\hline $\mathrm{H}$ & 4.6556354 .8686380 .251577 \\
\hline $\mathrm{O}$ & 6.4287885 .1727910 .525949 \\
\hline $\mathrm{H}$ & 6.6216836 .1296220 .552629 \\
\hline $\mathrm{H}$ & 7.1631874 .7062221 .008279 \\
\hline $\mathrm{H}$ & $-6.2887095 .935358-8.141814$ \\
\hline $\mathrm{H}$ & $-4.454784-0.792463-8.367642$ \\
\hline $\mathrm{H}$ & $-4.7260680 .022638-5.766546$ \\
\hline $\mathrm{H}$ & $-2.7220583 .927197-2.818522$ \\
\hline $\mathrm{H}$ & $-1.2683046 .260975-3.376180$ \\
\hline $\mathrm{H}$ & $1.5362707 .899430-7.497251$ \\
\hline $\mathrm{H}$ & $2.3325076 .859102-9.847532$ \\
\hline $\mathrm{H}$ & $-0.0945043 .171743-13.060375$ \\
\hline $\mathrm{H}$ & $-1.9036151 .158729-12.694790$ \\
\hline $\mathrm{H}$ & -0.3023641 .4085261 .259715 \\
\hline $\mathrm{H}$ & $2.648950-0.854749-2.785728$ \\
\hline
\end{tabular}

\footnotetext{
${ }^{2} \mathrm{TS}_{\mathrm{OO}} \mathrm{H}$
}

\begin{tabular}{|c|c|c|c|}
\hline $\mathrm{N}$ & $-3.1265144 .671862-8.480130$ & $\mathrm{~N}$ & 2.6985382 .2116040 .319575 \\
\hline $\mathrm{C}$ & $-4.3781004 .644073-7.864388$ & $\mathrm{H}$ & $2.8508361 .289388-0.080130$ \\
\hline $\mathrm{H}$ & $-4.5791223 .992559-7.033792$ & $\mathrm{H}$ & 3.3175583 .0287660 .120489 \\
\hline $\mathrm{O}$ & $-0.0004872 .585517-7.511393$ & $\mathrm{C}$ & $2.178342-0.094154-2.081147$ \\
\hline $\mathrm{O}$ & $1.1405033 .255797-6.305112$ & $\mathrm{O}$ & $2.145593-0.384907-0.826407$ \\
\hline $\mathrm{H}$ & $1.5222293 .855477-6.970459$ & $\mathrm{O}$ & $1.7726561 .041825-2.551640$ \\
\hline FE & $-1.5529093 .590629-7.950061$ & $\mathrm{H}$ & $-0.1232481 .750770-7.015592$ \\
\hline $\mathrm{N}$ & $-2.7482702 .001463-7.594401$ & $\mathrm{O}$ & $0.4044373 .223052-1.939683$ \\
\hline $\mathrm{N}$ & $-1.8019354 .193214-6.021484$ & $\mathrm{H}$ & $0.8399192 .325701-2.054458$ \\
\hline $\mathrm{N}$ & $-0.2732045 .146105-8.309260$ & $\mathrm{H}$ & $-0.2996943 .356688-2.601555$ \\
\hline $\mathrm{N}$ & $-1.3456003 .014872-9.873782$ & $\mathrm{O}$ & $2.9121022 .603749-4.290582$ \\
\hline $\mathrm{C}$ & $-3.1812401 .057528-8.512811$ & $\mathrm{H}$ & $2.4391261 .940022-3.693151$ \\
\hline $\mathrm{C}$ & $-4.0544780 .081043-7.865951$ & $\mathrm{H}$ & $2.3693172 .811975-5.087878$ \\
\hline $\mathrm{C}$ & $-4.1868630 .459226-6.560058$ & $\mathrm{O}$ & $6.5401794 .729758-2.467255$ \\
\hline $\mathrm{C}$ & $-3.3669421 .654663-6.387279$ & $\mathrm{H}$ & $6.4719204 .796947-1.491882$ \\
\hline $\mathrm{C}$ & $-2.4790893 .541632-5.015555$ & $\mathrm{H}$ & $6.0516253 .931700-2.801358$ \\
\hline $\mathrm{C}$ & $-2.3727844 .278272-3.758369$ & $\mathrm{O}$ & $2.5192854 .760623-2.491578$ \\
\hline $\mathrm{C}$ & $-1.6389145 .408708-4.015995$ & $\mathrm{H}$ & $2.9159824 .122550-3.135586$ \\
\hline $\mathrm{C}$ & $-1.2701175 .335788-5.440163$ & $\mathrm{H}$ & $1.6626254 .329613-2.188820$ \\
\hline $\mathrm{C}$ & $0.0306866 .154985-7.425609$ & $\mathrm{O}$ & $5.3486362 .524674-3.387442$ \\
\hline $\mathrm{C}$ & $1.0503747 .034993-7.986767$ & $\mathrm{H}$ & $5.8586411 .712350-3.534558$ \\
\hline $\mathrm{C}$ & $1.4206426 .508241-9.197800$ & $\mathrm{H}$ & $4.4769762 .538761-3.876760$ \\
\hline $\mathrm{C}$ & $0.5336425 .360436-9.424817$ & $\mathrm{O}$ & $3.7153694 .630036-0.078771$ \\
\hline $\mathrm{C}$ & $-0.5530603 .608357-10.842379$ & $\mathrm{H}$ & $3.4380324 .828499-1.024592$ \\
\hline $\mathrm{C}$ & $-0.7427072 .948580-12.129362$ & $\mathrm{H}$ & 4.6589904 .8492840 .130933 \\
\hline $\mathrm{C}$ & $-1.6474071 .941731-11.935521$ & $\mathrm{O}$ & 6.4253905 .1639120 .410282 \\
\hline $\mathrm{C}$ & $-2.0128361 .971137-10.521104$ & $\mathrm{H}$ & 6.6245896 .1196380 .427932 \\
\hline $\mathrm{C}$ & $-2.8493811 .072636-9.873032$ & $\mathrm{H}$ & 7.1508344 .7006400 .907531 \\
\hline $\mathrm{H}$ & $-3.2864800 .291003-10.485351$ & $\mathrm{H}$ & $-6.1877365 .899340-8.153147$ \\
\hline $\mathrm{C}$ & $-3.2131722 .361540-5.203543$ & $\mathrm{H}$ & $-4.515433-0.762594-8.379625$ \\
\hline $\mathrm{H}$ & $-3.7365121 .963268-4.341276$ & $\mathrm{H}$ & $-4.7704850 .004791-5.759470$ \\
\hline $\mathrm{C}$ & $-0.4675346 .246379-6.121249$ & $\mathrm{H}$ & $-2.7698343 .895539-2.818192$ \\
\hline $\mathrm{H}$ & $-0.1093507 .097138-5.556574$ & $\mathrm{H}$ & $-1.3373196 .239025-3.377496$ \\
\hline $\mathrm{C}$ & $0.3309294 .666197-10.614810$ & $\mathrm{H}$ & $1.4401037 .900815-7.451482$ \\
\hline $\mathrm{H}$ & $0.8718275 .007845-11.487048$ & $\mathrm{H}$ & $2.2522376 .867118-9.804202$ \\
\hline $\mathrm{N}$ & 0.6411741 .4113680 .952657 & $\mathrm{H}$ & $-0.1936813 .228161-13.028513$ \\
\hline $\mathrm{H}$ & 0.9414040 .5726250 .441597 & $\mathrm{H}$ & $-2.0104541 .224083-12.671215$ \\
\hline $\mathrm{C}$ & 1.5059882 .4760050 .854713 & $\mathrm{H}$ & -0.3033221 .4071381 .281108 \\
\hline $\mathrm{N}$ & 1.2592113 .7281181 .273563 & $\mathrm{H}$ & $2.585787-0.825160-2.779492$ \\
\hline $\mathrm{H}$ & 0.3460894 .0597181 .591745 & & \\
\hline $\mathrm{H}$ & 1.9890874 .4115541 .060591 & & \\
\hline
\end{tabular}




\begin{tabular}{|c|c|}
\hline $\mathrm{V}$ & $-4.4395396 .166855-9.468333$ \\
\hline $\mathrm{H}$ & $-4.7179136 .899175-10.128520$ \\
\hline $\mathrm{C}$ & $-5.2074005 .575737-8.462612$ \\
\hline $\mathrm{C}$ & $-3.1973815 .617251-9.447362$ \\
\hline $\mathrm{H}$ & $-2.4024525 .913286-10.108002$ \\
\hline N & $-3.1294474 .690909-8.482113$ \\
\hline $\mathrm{C}$ & $-4.3796564 .656040-7.863244$ \\
\hline $\mathrm{H}$ & $-4.5748624 .002930-7.032308$ \\
\hline $\mathrm{O}$ & $-0.0104282 .619131-7.558007$ \\
\hline o & $1.3871233 .532343-6.122986$ \\
\hline $\mathrm{H}$ & $1.7551663 .792635-6.987164$ \\
\hline $\mathrm{FE}$ & $-1.5247753 .596884-7.960113$ \\
\hline N & $-2.7443932 .012927-7.606524$ \\
\hline $\mathrm{N}$ & $-1.7771694 .189057-6.035809$ \\
\hline $\mathrm{N}$ & $-0.2767625 .163603-8.333668$ \\
\hline $\mathrm{N}$ & $-1.3573013 .049472-9.911862$ \\
\hline $\mathrm{C}$ & $-3.1638911 .071137-8.517653$ \\
\hline $\mathrm{C}$ & $-4.0229950 .079072-7.872993$ \\
\hline C & $-4.1497840 .447110-6.563438$ \\
\hline $\mathrm{C}$ & $-3.3431881 .653087-6.392684$ \\
\hline $\mathrm{C}$ & $-2.4441603 .541793-5.028293$ \\
\hline $\mathrm{C}$ & $-2.3348834 .279085-3.768504$ \\
\hline $\mathrm{C}$ & $-1.6101855 .412972-4.028474$ \\
\hline $\mathrm{C}$ & $-1.2450135 .343230-5.456401$ \\
\hline $\mathrm{C}$ & $0.0380646 .155256-7.456003$ \\
\hline $\mathrm{C}$ & $1.0689077 .034956-8.009968$ \\
\hline $\mathrm{C}$ & $1.4410416 .511182-9.219010$ \\
\hline $\mathrm{C}$ & $0.5399105 .371131-9.458853$ \\
\hline $\mathrm{C}$ & $-0.5733653 .630280-10.870832$ \\
\hline $\mathrm{C}$ & $-0.7511742 .965950-12.158840$ \\
\hline $\mathrm{C}$ & $-1.6488201 .953203-11.962022$ \\
\hline $\mathrm{C}$ & $-2.0179511 .989254-10.546158$ \\
\hline $\mathrm{C}$ & 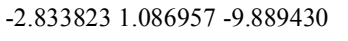 \\
\hline $\mathrm{H}$ & $-3.2636140 .295043-10.494013$ \\
\hline $\mathrm{C}$ & $-3.1798662 .353411-5.210768$ \\
\hline $\mathrm{H}$ & $-3.6889111 .950244-4.342591$ \\
\hline $\mathrm{C}$ & $-0.4619686 .252769-6.141659$ \\
\hline $\mathrm{H}$ & $-0.1009397 .105508-5.582037$ \\
\hline $\mathrm{C}$ & $0.3305594 .690732-10.640514$ \\
\hline $\mathrm{H}$ & $0.8830665 .016910-11.511465$ \\
\hline $\mathrm{N}$ & $\begin{array}{llll}0.653082 & 1.414890 & 0.940904\end{array}$ \\
\hline
\end{tabular}

$$
\begin{array}{lllll}
\mathrm{H} & 0.9548140 .5737310 .433018 & \mathrm{H} & -2.0078981 .230525-12.694732 \\
\mathrm{C} & 1.5196602 .4781780 .844037 & \mathrm{H} & -0.2894401 .4131261 .275003 \\
\mathrm{~N} & 1.2717303 .7323411 .257220 & \mathrm{H} & 2.623457-0.855895-2.749773
\end{array}
$$

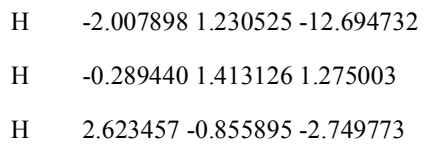$$
\mathrm{H} \quad 0.3601554 .0628101 .578983
$$$$
\text { H } \quad 2.0039074 .4143961 .042386
$$$$
\mathrm{N} \quad 2.7132432 .2118350 .311876
$$$$
\mathrm{H} \quad 2.8702731 .288362-0.082964
$$$$
\text { H } \quad 3.3326963 .0268580 .116027
$$$$
\text { C } \quad 2.207914-0.118346-2.063179
$$$$
\text { O } 2.155922-0.399422-0.805841
$$$$
\text { O } \quad 1.8084501 .012977-2.547720
$$$$
\text { H } \quad-0.1397411 .915457-6.897593
$$$$
\text { O } \quad 0.4635233 .216193-1.984469
$$$$
\text { H } \quad 0.8951832 .313747-2.081210
$$$$
\text { H } \quad-0.2234263 .347761-2.663689
$$$$
\text { O } \quad 3.0607002 .572334-4.223865
$$$$
\text { H } \quad 2.5465611 .914395-3.659066
$$$$
\mathrm{H} \quad 2.5289502 .857516-5.010280
$$$$
\text { O } 6.4892914 .616872-2.366333
$$$$
\text { H } \quad 6.4531464 .744274-1.394939
$$$$
\text { H } \quad 6.0503153 .763037-2.626112
$$$$
\text { O } \quad 2.5862704 .759515-2.500331
$$$$
\text { H } \quad 3.0208984 .109751-3.108961
$$$$
\text { H } \quad 1.7266004 .320068-2.218501
$$$$
\text { O } \quad 5.4046162 .304577-3.162934
$$$$
\text { H } \quad 6.0048781 .608867-3.476969
$$$$
\text { H } \quad 4.5823122 .399021-3.726579
$$$$
\text { O } \quad 3.7126914 .650606-0.056410
$$$$
\text { H } \quad 3.4592024 .845426-1.010182
$$$$
\mathrm{H} \quad 4.6541004 .8601700 .170423
$$$$
\text { O } \quad 6.4190585 .1670690 .478710
$$$$
\mathrm{H} \quad 6.6163306 .1231010 .497626
$$$$
\text { H } \quad 7.1480374 .7024420 .969926
$$$$
\text { H } \quad-6.1981725 .901181-8.145555
$$$$
\text { H } \quad-4.485485-0.762678-8.388382
$$$$
\text { H } \quad-4.721910-0.016705-5.759938
$$$$
\text { H } \quad-2.7228843 .894066-2.825486
$$$$
\text { H } \quad-1.3090776 .244433-3.391235
$$$$
\mathrm{H} \quad 1.4628947 .893824-7.466656
$$$$
\text { H } \quad 2.2793996 .863187-9.820105
$$$$
\text { H } \quad-0.1993013 .245857-13.056144
$$

\section{${ }^{2} \mathbf{C}_{\mathrm{Ib}} \mathbf{H}$}

$$
\begin{aligned}
& \text { N } \quad-4.4398216 .178648-9.469048 \\
& \mathrm{H} \quad-4.7112136 .917119-10.125425 \\
& \text { C } \quad-5.2199545 .576823-8.479932 \\
& \text { C } \quad-3.1993215 .624727-9.441732 \\
& \text { H } \quad-2.3960835 .926740-10.089886 \\
& \mathrm{~N} \quad-3.1465004 .683057-8.491052 \\
& \text { C } \quad-4.4032164 .643502-7.886607 \\
& \text { H } \quad-4.6091433 .981885-7.065176 \\
& \text { O } \quad-0.0662622 .601827-7.482833 \\
& \text { O } \quad 1.8470993 .724896-6.127098 \\
& \text { H } \quad 1.0953933 .283598-6.661883 \\
& \text { FE } \quad-1.5620933 .563180-7.968190 \\
& \text { N } \quad-2.7932301 .989719-7.613625 \\
& \mathrm{~N} \quad-1.8188744 .181745-6.045466 \\
& \text { N } \quad-0.2816825 .118641-8.323950 \\
& \mathrm{~N} \quad-1.3949763 .011734-9.902325 \\
& \text { C } \quad-3.2159641 .044933-8.516148 \\
& \text { C } \quad-4.0832610 .061711-7.866960 \\
& \text { C } \quad-4.2156470 .443047-6.562415 \\
& \text { C } \quad-3.4003571 .646922-6.397521 \\
& \text { C } \quad-2.4892633 .537774-5.039698 \\
& \text { C } \quad-2.3637884 .267350-3.775925 \\
& \text { C } \quad-1.6228825 .392139-4.030804 \\
& \text { C } \quad-1.2620005 .325864-5.459832 \\
& \text { C } \quad 0.0401156 .112905-7.456000 \\
& \text { C } \quad 1.0762916 .987365-8.014047 \\
& \text { C } \quad 1.4476216 .454118-9.217106 \\
& \text { C } \quad 0.5385845 .315782-9.450298 \\
& \text { C } \quad-0.5968873 .582785-10.854402 \\
& \text { C } \quad-0.7702642 .917905-12.142305 \\
& \text { C } \quad-1.6770621 .912457-11.951266 \\
& \text { C } \quad-2.0590881 .951583-10.539478 \\
& \text { C } \quad-2.8797771 .052986-9.888206 \\
& \text { H } \quad-3.3078290 .259901-10.492250 \\
& \text { C } \quad-3.2363062 .353950-5.221681 \\
& \text { H } \quad-3.7522361 .961118-4.352988
\end{aligned}
$$




\begin{tabular}{|c|c|}
\hline 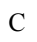 & $-0.4582486 .219757-6.140572$ \\
\hline $\mathrm{H}$ & $-0.0790757 .063030-5.578677$ \\
\hline $\mathrm{C}$ & $0.3217254 .634751-10.626071$ \\
\hline I & $0.8798414 .947765-11.497803$ \\
\hline J & 0.6543721 .4320910 .906953 \\
\hline $\mathrm{H}$ & 0.9530260 .5999560 .379960 \\
\hline $\mathrm{C}$ & 1.5239722 .4944210 .829349 \\
\hline J & 1.2804133 .7414971 .264946 \\
\hline $\mathrm{H}$ & 0.3680014 .0695481 .587053 \\
\hline $\mathrm{H}$ & 2.0189654 .4235571 .068002 \\
\hline $\mathrm{N}$ & 2.7187712 .2347000 .295119 \\
\hline $\mathrm{H}$ & $2.8808071 .319174-0.113896$ \\
\hline $\mathrm{H}$ & 3.3422413 .0502260 .123049 \\
\hline $\mathrm{C}$ & $2.177984-0.070204-2.104245$ \\
\hline $\mathrm{O}$ & $2.118956-0.360748-0.850313$ \\
\hline $\mathrm{O}$ & $1.7848971 .065205-2.587565$ \\
\hline $\mathrm{H}$ & $-0.2537431 .712984-7.130153$ \\
\hline $\mathrm{O}$ & $0.5108303 .293632-2.014966$ \\
\hline $\mathrm{H}$ & $0.9109842 .376238-2.115748$ \\
\hline $\mathrm{H}$ & $-0.2081863 .437881-2.657101$ \\
\hline $\mathrm{O}$ & $3.2705992 .598602-4.096335$ \\
\hline $\mathrm{H}$ & $2.6538411 .969683-3.608950$ \\
\hline $\mathrm{H}$ & $2.8612872 .959401-4.918354$ \\
\hline $\mathrm{O}$ & $6.4614304 .530247-2.250876$ \\
\hline $\mathrm{H}$ & $6.4457774 .733331-1.291588$ \\
\hline $\mathrm{H}$ & $6.0759543 .628705-2.421108$ \\
\hline $\mathrm{O}$ & $2.6630634 .814407-2.452914$ \\
\hline $\mathrm{H}$ & $3.1571304 .164269-3.012949$ \\
\hline $\mathrm{H}$ & $1.7940004 .365736-2.215398$ \\
\hline $\mathrm{O}$ & $5.4774472 .114997-2.863840$ \\
\hline $\mathrm{H}$ & $6.1269421 .475565-3.200925$ \\
\hline I & $4.7159702 .278148-3.495352$ \\
\hline ) & 3.7158314 .6923840 .014689 \\
\hline$H$ & $3.4769344 .902912-0.940461$ \\
\hline$H$ & 4.6569494 .8879640 .252755 \\
\hline $\mathrm{O}$ & 6.4307255 .1796450 .555161 \\
\hline$H$ & 6.6290606 .1354490 .580904 \\
\hline$H$ & 7.1651194 .7079441 .032054 \\
\hline$H$ & $-6.2092065 .905958-8.161941$ \\
\hline$H$ & $-4.544100-0.782776-8.379343$ \\
\hline & $-4.791131-0.012313-5.7564$ \\
\hline
\end{tabular}

$$
\begin{array}{ll}
\mathrm{H} & -2.7510043 .882265-2.832612 \\
\mathrm{H} & -1.3081386 .216283-3.390657 \\
\mathrm{H} & 1.4715447 .846904-7.472720 \\
\mathrm{H} & 2.2885336 .797431-9.819659 \\
\mathrm{H} & -0.2126213 .192691-13.037623 \\
\mathrm{H} & -2.0379011 .194911-12.688145 \\
\mathrm{H} & -0.2855171 .4263071 .248344 \\
\mathrm{H} & 2.594265-0.802114-2.796404
\end{array}
$$$$
{ }^{2} \mathbf{C}_{\mathrm{R}} \text { Aspp136 }
$$$$
\mathrm{N} \quad-4.3686736 .100681-9.489327
$$$$
\text { H } \quad-4.6539146 .832923-10.150427
$$$$
\text { C } \quad-5.0775055 .596623-8.394995
$$$$
\text { C } \quad-3.1568315 .479586-9.544059
$$$$
\text { H } \quad-2.4243465 .670326-10.309974
$$$$
\text { N } \quad-3.0527294 .604128-8.532865
$$$$
\text { C } \quad-4.2450244 .670661-7.815030
$$$$
\text { H } \quad-4.4214044 .034603-6.969580
$$$$
\text { O } \quad-0.1137462 .270193-7.537622
$$$$
\text { O } \quad 1.0798592 .941206-7.008978
$$$$
\text { H } \quad 1.0043813 .807762-7.466881
$$$$
\text { FE } \quad-1.4708913 .434604-8.037069
$$$$
\text { N } \quad-2.7102671 .866513-7.723466
$$$$
\mathrm{N} \quad-1.6704364 .016533-6.095817
$$$$
\mathrm{N} \quad-0.1677115 .002762-8.367771
$$$$
\mathrm{N} \quad-1.2894792 .895952-9.972064
$$$$
\text { C } \quad-3.1073280 .910006-8.641139
$$$$
\text { C } \quad-3.991250-0.065580-7.998867
$$$$
\text { C } \quad-4.1309960 .321595-6.695713
$$$$
\text { C } \quad-3.3108711 .524039-6.525726
$$$$
\text { C } \quad-2.3809603 .350871-5.110583
$$$$
\text { C } \quad-2.2232794 .036446-3.826121
$$$$
\text { C } \quad-1.3878855 .101698-4.036389
$$$$
\text { C } \quad-1.0683385 .095613-5.473561
$$$$
\text { C } \quad 0.1520196 .005022-7.458458
$$$$
\text { C } \quad 1.0642406 .958172-8.071285
$$$$
\text { C } \quad 1.3067936 .540195-9.353972
$$$$
\text { C } \quad 0.4787185 .346364-9.559376
$$$$
\text { C } \quad-0.5680643 .561051-10.950223
$$$$
\text { C } \quad-0.7372212 .896045-12.239896
$$$$
\text { C } \quad-1.5461901 .818112-12.025036
$$

C $\quad-1.8990291 .822492-10.607940$

C $\quad-2.7234440 .897874-9.978075$

H $\quad-3.1196210 .095899-10.590764$

C $\quad-3.1385432 .200901-5.322575$

H $\quad-3.6484501 .774139-4.466555$

C $\quad-0.2663646 .024902-6.135040$

H $\quad 0.1084186 .852521-5.543632$

C $\quad 0.2323364 .684373-10.756571$

H $\quad 0.6447315 .115011-11.658015$

$\mathrm{N} \quad 0.3413721 .3843100 .811778$

H $\quad 0.8748700 .5099330 .840072$

C $\quad 1.0023972 .5102120 .347093$

$\mathrm{N} \quad 0.6382583 .7543130 .561812$

H $\quad-0.1484434 .0061101 .177503$

$\mathrm{H} \quad 1.2024634 .6240780 .150270$

N $\quad 2.0847282 .253018-0.427036$

H $2.3568291 .307593-0.664235$

H $\quad 2.6692653 .008200-0.764382$

C $\quad 2.517863-1.629361-2.380014$

O $\quad 2.317055-2.300817-1.367173$

O $\quad 2.310687-0.262781-2.436204$

H $\quad 1.3758900 .135194-2.497476$

O $\quad 0.1034861 .213214-2.742106$

H $\quad-0.7521330 .793931-2.977690$

H $\quad-0.0432451 .942598-2.112060$

O $\quad 3.6981961 .903757-3.379178$

H $\quad 2.9628582 .351399-3.897214$

H $\quad 3.4218941 .003414-3.110453$

O $\quad 4.9514643 .779976-2.147033$

$\mathrm{H} \quad 4.5850332 .939555-2.582100$

H $\quad 5.2203603 .623132-1.202072$

O $\quad 1.8653555 .752665-0.344981$

H $\quad 1.5232086 .611644-0.006679$

H $\quad 2.1514545 .679780-1.321232$

O $\quad 1.5669553 .037139-4.397975$

H $\quad 0.8723172 .491531-3.969202$

H $\quad 1.3616423 .092528-5.380600$

O $\quad 2.6985215 .081005-2.630262$

H $\quad 2.2141504 .543859-3.299840$

H $\quad 3.6353224 .739222-2.489995$

H $\quad-6.0619525 .920027-8.056853$ 


$$
\begin{array}{ll}
\mathrm{H} & -4.412572-0.938177-8.497995 \\
\mathrm{H} & -4.710834-0.129895-5.890721 \\
\mathrm{H} & -2.7860883 .756415-2.935675 \\
\mathrm{H} & -0.9574695 .835623-3.355094 \\
\mathrm{H} & 1.5117497 .776455-7.507179 \\
\mathrm{H} & 2.0134237 .012786-10.036174 \\
\mathrm{H} & -0.2936423 .259976-13.166642 \\
\mathrm{H} & -1.9462611 .133132-12.772574 \\
\mathrm{H} & -0.5485941 .3322531 .264795 \\
\mathrm{H} & 2.931651-2.104759-3.269307
\end{array}
$$

$$
\begin{aligned}
& { }^{2} \mathbf{P} \text { Aspp } 136 \\
& \text { N } \quad-4.3439326 .099156-9.486679 \\
& \text { H } \quad-4.6278936 .831528-10.147770 \\
& \text { C } \quad-5.0543565 .599635-8.391346 \\
& \text { C } \quad-3.1390415 .465763-9.544587 \\
& \mathrm{H} \quad-2.4105415 .656183-10.313273 \\
& \text { N } \quad-3.0337824 .585253-8.535727 \\
& \text { C } \quad-4.2267864 .667416-7.815417 \\
& \mathrm{H} \quad-4.4115424 .034510-6.970580 \\
& \text { O } \quad-0.1362272 .315098-7.631501 \\
& \text { O } \quad 1.3637003 .983295-6.026997 \\
& \text { H } \quad 0.9286303 .287319-6.598243 \\
& \text { FE } \quad-1.4027723 .279391-8.061890 \\
& \mathrm{~N} \quad-2.7615031 .820459-7.732370 \\
& \text { N } \quad-1.6284863 .866875-6.096527 \\
& \mathrm{~N} \quad-0.1348144 .847629-8.426207 \\
& \text { N } \quad-1.3175202 .825774-10.010325 \\
& \text { C } \quad-3.1121090 .828968-8.629477 \\
& \text { C } \quad-4.022460-0.118508-7.993303 \\
& \text { C } \quad-4.2282420 .320117-6.712944 \\
& \text { C } \quad-3.4304391 .529351-6.548867 \\
& \text { C } \quad-2.5277603 .374008-5.139897 \\
& \text { C } \quad-2.3169354 .058480-3.882274 \\
& \text { C } \quad-1.2487434 .915166-4.058678 \\
& \text { C } \quad-0.8423934 .753411-5.437742 \\
& \text { C } \quad 0.3972315 .633378-7.466880 \\
& \text { C } \quad 1.1751106 .697267-8.030857 \\
& \text { C } \quad 1.2177856 .489327-9.393493 \\
& \text { C } \quad 0.3679095 .334161-9.650257 \\
& \text { C } \quad-0.6866923 .542266-11.017829
\end{aligned}
$$$$
\text { C } \quad-1.8966761 .723867-10.620028
$$$$
\text { C } \quad-2.6847660 .781213-9.958869
$$$$
\text { H } \quad-3.051836-0.048304-10.553252
$$$$
\text { C } \quad-3.3409772 .270109-5.371919
$$$$
\mathrm{H} \quad-3.9227001 .899044-4.534828
$$

$$
\mathrm{C}
$$

O $3.8677032 .058072-3.007096$

$\mathrm{H} \quad 3.0470892 .544165-3.328107$

H $\quad 3.7098081 .094610-2.977573$

O $\quad 5.2277603 .874953-1.840718$

H $\quad 4.8201063 .058366-2.288426$

H $\quad 5.4833653 .662735-0.903265$

O 2.0398395 .8998410 .033702

$\mathrm{H} \quad 1.6303626 .7486830 .328258$

H $\quad 2.4615175 .835774-0.910953$

O $\quad 1.7169303 .353948-3.562402$

H $\quad 0.9809392 .848873-3.156674$

$\mathrm{H} \quad 1.5401113 .565144-4.544109$

O $\quad 3.0356895 .312179-2.167715$

H $\quad 2.4751744 .693422-2.706645$

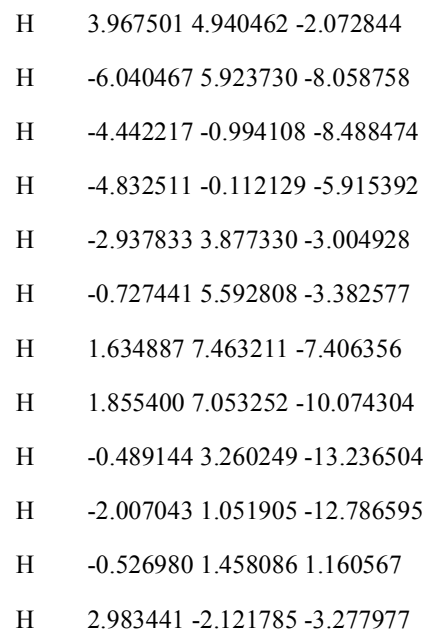

